\title{
Organic Chemistry Section:
}

\section{Summary of Activities July 1969 to June 1970}

\begin{abstract}
U.S. ARTMENT OF JMMERCE National Bureau of indards
\end{abstract}





\section{Maurice H. Stans, Secretary \\ U,, NATIONAL BUREAU OF STANDARDS - Lewis M. Branscomb, Director is o

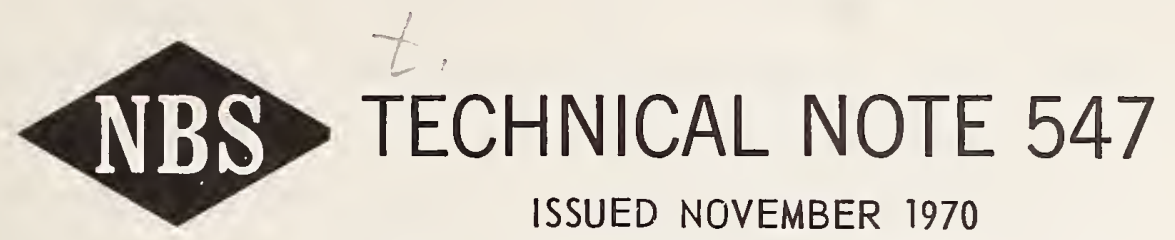 \\ Nat. Bur. Stand. (U.S.), Tech. Note 547, 134 pages (Nov. 1970) CODEN: NBTNA}

UNITED STATES DEPARTMENT OF COMMERCE

\section{Organic Chemistry Section:}

\section{Summary of Activities July 1969 to June 1970}

Robert Schaffer, Editor

Organic Chemistry Section Analytical Chemistry Division Institute for Materials Research National Bureau of Standards

Washington, D.C. 20234

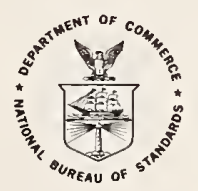

NBS Technical Notes are designed to supplement the Bureau's regular publications program. They provide a means for making available scientific data that are of transient or limited interest. Technical Notes may be listed or referred to in the open literature.

For sale by the Superintendent of Documents, U.S. Government Printing Office, Washington, D.C., 20402. (Order by SD Catalog No. C 13.46:547), Price $\$ 1.25$. 
The Analytical Chemistry Division was established as a separate division at the National Bureau of Standards on September 1, 1963, and became part of the Institute for Materials Research in the February l, 1964, reorganization. It consists at present of nine sections and about 100 technical personnel encompassing some 60 different analytical competences from activation analysis and atomic absorption to vacuum fusion and $x$-ray spectroscopy. These competences, and in turn the sections which they comprise, are charged with research at the forefront of analysis as well as awareness of the practical sample, be it standard reference material or service analysis. In addition it is their responsibility to inform others of their efforts.

Formal publication in scientific periodicals is a highly important output of our laboratories. In addition, however, it has been our experience that informal, annual summaries of progress describing efforts of the past year can be very valuable in disseminating information about our programs. A word is perhaps in order about the philosophy of these yearly progress reports. In any research program a large amount of information is obtained and techniques developed which never find their way into the literature. This includes the "negative results" which are so disappointing and unspectacular but which can often save others considerable work. Of importance also are the numerous small items which are often explored in a few days and which are not important enough to warrant publication-yet can be of great interest and use to specialists in a given area. Finally there are the experimental techniques and procedures, the designs and modifications of equipment, etc., which often require months to perfect and yet all too often must be covered in only a line or two of a journal article. 
Thus our progress reports endeavor to present this information which we have struggled to obtain and which we feel might be of some help to others. Certain areas which it appears will not be treated fully in regular publications are considered in some detail here. Other results which are being written up for publication in the journal literature are covered in a much more abbreviated form.

At the National Bureau of Standards publications such as these fit logically into the category of a Technical Note. In 1970 we plan to issue these summaries for all of our sections. The following is the sixth annual report on progress of the Organic Chemistry Section.

W. Wayne Meinke, Chief Analytical Chemistry Division 
This is the sixth annual progress report of the Organic Chemistry Section of the Analytical Chemistry Division, and it describes the activities of the Section from July 1,1969 to June 30, 1970. Unlike the formal, journal publications that emanate from the section from time to time, this report summarizes the activities of the entire Organic Chemistry Section during that period.

Following the established pattern within this Division; the programs of the Section have both a research and a sample aspect: about half of the total effort of the section has been applied to research, and the remainder to standard Reference Materials (SRMs). The Section must concern itself with many subjects in organic chemistry in supporting the National Bureau of Standards' overall mission, which is to contribute, where possible, to the solution of nationwide problems through the enhancement of meaningful measurement. To that end, in the past year, much of the Section's effort was directed to developing SRMs needed in clinical chemistry laboratories. This has entailed working with organic compounds that fall into a number of classical areas of organic chemistry. To maintain overall unity in the Section's program, considerable attention is given to one major area of organic chemistry, the carbohyärates, developing therein the Section's competences in organic analysis, characterization, and synthesis. These competences are utilized to the maximum extent for the development of SRMS. Our efforts in serving clinical chemistry have been fostered by the National Institute of General Medical Services of the National Institutes of Health. 
In this report, in order to describe procedures

adequately, it has occasionally been necessary to identify commercial equipment. In no case does such identification imply NBS recommendation or endorsement, nor does it imply that the equipment identified is necessarily the best available for the purpose.

Robert Schaffer, Chief

Organic Chemistry Section 
1. CLINICAL STANDARDS • • . . • • . . . . . . . . . 2

Cholesterol. Post-issuance Studies. . . . . . . 3

Thin-layer Chromatography of Cholesterol

Samples

Liebermann-Burchard Analysis

Differential Scanning Calorimetry (DSC)

and Phase-solubility Analysis (PSA). . . . . 4

Urea (Clinical). Post-issuance Studies. . . . . . 5

Determination of Purity of Urea by

Differential Scanning Calorimetry. . . . . . 5

Determination of Moisture Content of Urea

by Karl Fischer Titration. . . . . . . . . 5

Determination of Purity of Urea by Phase-

solubility Analysis. . . . . . . . . . . 6

Bilirubin.

Standard Solutions of Bilirubin . . . . . . . 6

Some Estimates of the Millimolar

Absorptivities of Bilirubin in

Non-aqueous Solvents . . . . . . . 10

Millimolar Absorptivities in Methyl Sulfoxide for Bilirubin Diazo-coupled by an EvelynMalloy Procedure . . . . . . . • . . . 12

Millimolar Absorptivities for Bilirubin

Diazo-coupled by the Jendrassik-Grof

Procedure. •. . . . . . . . . . 14

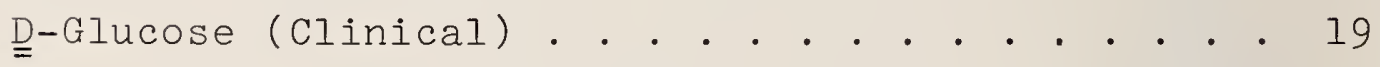

Analysis of D-Glucose by Gas-liquid

Chromatograp̄hy . . . . . . . • . . . . . . 19.

Apparent Purity of D-Glucose by

Differential Scannīing Calorimetry. . . . . . 22

Determination of Moisture Content of

D-Glucose Samples. 
Cortisol

Physical Properties of Some Cortisol

Samples. . . . . . . . . . . . . 25

Determination of Purity by Differential

Scanning Calorimetry . . . . . . . 26

Spectrometric Properties. . . . . . . . 27

Thin-layer Chromatography of Cortisol . . 28

4-Hydroxy-3-methoxy-DL-mandélic Acid (VMA) . . 30

Melting Point of Commercial Samples of

VMA. . . . . . . . . . . . . . 30

Thin-layer Chromatography of Commercial

Samples of VMA . . . . . . . . . . . 31

Spectrometric Properties. . . . . . . . . 32

Examination by Gas-liquid Chromatography

(G.I.c.) of the Per(trimethylsilyl)

Derivatives of Samples of VMA. . . . . . 37

Determination of Purity of VMA by

Differential Scanning Calorimetry. . . . 37

D-Mannitol (Clinical). . . . . . . . . . 38

Thin-layer Chromatography (T.I.c.) of

D-Mannitol . . . . . . . . . . . . . . . 38

Pāper Chromatography of D-Mannitol. . . . . 39

Optical Rotation of Samples of
D-Mannitol. . . . . . . . . . . 40

$\beta$-Nicotinamide Adenine Dinucleotide $(\beta-N A D)$ and

its 1,4-Reduced Form $(\beta-\mathrm{NADH}) . . . . . .942$

Loss of Weight on Drying. . . . . . . . 45

Elemental Analysis. . . . . . . . . . . 45

Paper Chromatography of $\beta-\mathrm{NAD} . .2 .0 .946$

Gas-liquid Chromatography of Commercial

B-NAD after Per(trimethylsilyl)ation . . . 51

Spectrometric Methods... . . . . . . 52

Enzymic Assay of B-NAD. . . . . . . . . . 53

2. CARBOHYDRATE SYNTHESIS, STRUCTURE, AND

CHARACTERIZATION: NOVEL RESEARCH MATERIALS

AND MODEL COMPOUNDS. . . . . . . . . . . . 55

Synthesis of the Isomeric Di-O-isopropylidene- $\beta-$ D-fructopyranoses.............. 56 
Synthesis of $\underline{\underline{D}}$-Psicose

Model Parameters for the Analysis of Skew

Conformations by P.m.r. Spectroscony.

The Conformations of $1,2: 4,6$-Di-o-benzylidene$\alpha-\underline{\underline{D}}-g i$ copyranose Derivatives

Iterative Analysis of the Proton Magnetic

Resonance Spectra of $1,2: 3,5-D i-0$-isopropylidene$\alpha-\underline{D}-g$ lucofuranose Derivatives

Cyclic Polyhydroxy Compounds

Determination of Inososes with an Alkaline Solution of Copper(II) Oxalate-Tartrate Complex (the Somogyi Reagent); Structure of the Reagent (by E.s.r. Studies), and Reaction Mechanisms Involved... . .

E.s.r. Evidence for a Dimer Structure of the Alkaline Copper(II) Tartrate Complex in the Fehling Reagent at $77 \mathrm{~K}$. . . . . . . 91 Preparation of Inositol Hexasulfate. . . . 94

3. GENERAL ORGANIC CHEMISTRY. . . . . . . . . . 95

Novel Iodination of the $\alpha$-Methylene Group in

1,3-Diphenyl-1,3-propanedione and Related

$\beta$-Diketones with Periodic Acid. . . . . . . . 95

Degradation of Bilirubin with Chromic Acid . . . 99

Degradation of Hemin and Related Bile Pigments with Periodic Acid. . . . . . . . . . . . 101

Isolation of a Biliverdine Type of Reaction Intermediate on Oxidation of Bilirubin with Periodic Acid............... 103

4. DETECTION AND IDENTIFICATION OF TRACES OF ORGANIC COMPOUNDS . . . . . . . . . . . . 105 2,4,6-Trinitrotoluene. . . . . . . . . . . 105 
Hexahydro-1,3,5-trinitro-s-triazine $\underset{\sim}{2}$ ). . . . . 106

Detection of 2 by Diazonium Coupling. . . . 106

Detection of $\underset{\sim}{2}$ by Formation of

Formaldehyde . . . . . . ...... 106

Detection of 2 by Formation of Hydrazine. . . 107

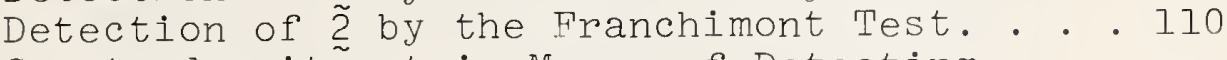

Spectrodensitometric Means of Detecting

Low Levels of $\underset{\sim}{2} \cdot$. . . . . . . . . . . 111

5. PERSONNEL AND ACTIVITIES . . . . . . . . . 113

Personnel Listing . . . . . . . . . . . 113

Publications. . . . . . . . . . . . . 113

Manuscripts in Process of Publication . . . 114

Lectures. . . . . . . . . . . . 116

6. ACKNOWLEDGMENTS. . . . . . . . . . . . . . 117

7. REFERENCES . . . . . . . . . . . . . . . 118

FIGURE NO.

LIST OF FIGURES

1. Determination of the purity of a volatile compound by gas-liquid chromatography. . . 20

2. Recording an optical rotatory

dispersion spectrum . . . . . . . . . . . .

3. P.m.r. spectra of $\mathrm{l}$ at $100 \mathrm{MHz}$; the signals of the aromatic protõns are not shown. (a)

Sirgle resonance spectrum, (b) theoretical spectrum from iterative analysis, (c) irradiation of $\mathrm{H}-\mathrm{l}$, and (d) irradiation of

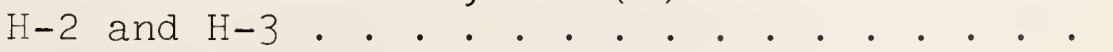

4. Adjustment of the strength of the magnetic field, at the console of a nuclear magnetic resonance spectrometer.

5. Positioning a sample tube, prior to insertion into the probe head of a nuclear magnetic resonance spectrometer. 
6. Partial p.m.r. spectra of 4 . (a) Observed spectrum of solution in pỹridine at $100 \mathrm{MHz}$, and (b) computed spectrum after iterative analysis as a seven-spin system . . . . .

7. Partial p.m.r. spectra of 1 . (a) Observed spectrum at $220 \mathrm{MHz}$, and (b) computed spectrum after iterative analysis as a seven-spin system . . . . . . . . . . . . .

8. Partial p.m.r. spectra of $1-{ }^{15} \mathrm{~N}$. (a) Observed spectrum of solution in benzene-d. 6 at $220 \mathrm{MHz}$, and (b) computed spectrum after iterative analyses of two- and six-spin systems . . . . . . . . . . . . . . . .

9. P.m.r. spectra of 2 . (a) Computed spectrum after iterative añalysis by magneticequivalence factoring, and $(b)$ observed spectrum of solution in $8: 1(\mathrm{v} / \mathrm{v})$ acetone- $d_{6}$ benzene at $90 \mathrm{MHz}$. . . . . . . . .

10. E.s.r. spectra of frozen (77 K) aqueous alkaline solutions of $\mathrm{Cu}$ (II) complexes: spectrum A, tartrate-oxalate (the Somogyi reagent); B, tartrate (the Fehling reagent); C, citrate (the Benedict reagent): and $D$, tetraamine hydrate (cuprammonium, the Reeves reagent). (All four low-field e.s.r. spectra on the left side of the dotted line were recorded at somewhat greater field-modulation amplitude than the spectra for the g 22 region.) . . . . . . . . . . .

LIST OF TABLES

TABLE NO.

1. Dependence of absorption on $\mathrm{pH}$, for "azobilirubin" in methyl sulfoxide. . . . . .

2. Millimolar absorptivities of bilirubin and "diazobilirubin" in human serum . . . . . . . 17

3. Milimolar absorptivity of bilirubin in human-serum albumin. 
4. Isomerization of D-glucose during melting to $\mathrm{T}_{\max } \cdot \cdot \cdot$

5. Effect of duration of heating at $0.625 \mathrm{~K} / \mathrm{min}$ on the apparent purity of D-glucose (clinical) as measured by differential scanning calorimetry . . . . . . . . . . . . . .

6. Percent of water in samples of D-glucose . . . 24

7. Some physical properties of samples of cortisol

8. T.I.c. data on commercial samples of cortisol

9. Melting points of samples of VMA . . . . . . 31

10. T.I.c. data on commercial samples of VMA. . . 33

11. Approximate percent of impurity in commercial samples of VMA as indicated by mass spectrometry

12. Re-estimation of purity of commercial samples of VMA by mass spectrometry .. . . . . . 35

13. Relative retention times and percentages of components of samples of per(trimethylsilyl)ated VMA and related compounds

14. Analysis of purity of VMA by differential scanning calorimetry

15. Thin-layer chromatography of D-mannitol . . . 39

16. Specific rotations, at various wavelengths, of solutions of commercial samples of D-mannitol in acidified ammonium molybdate solution . .

17. Ultraviolet absorption peaks of $\beta-N A D H$ at pH 10

18. Analysis of a commercial sample (lot no. 2331) of $\beta-N A D$ 
19. Paper chromatography of samples of commercial $\beta-N A D$. Detection by fluorescence-quenching or KOH-spraying, or both . . . . . . . . . .

20. Paper chromatography of B-NAD (lot no. 2331). . 50

21. Retention times of products obtained by per(trimethylsilyl)ation of commercial B-NAD (lot no. 2331). . . . . . . . . 51

22. Ultraviolet spectral data for B-NAD (lot no. 2331).............. . . 52

23. Enzymic assay of $\beta-N A D$ (lot no. 2331) by reduction with ethanol in the presence of horse-liver alcohol dehydrogenase . . . . . 54

24. Coupling constants $(\mathrm{Hz})$ of 6 -deoxy-1,2:3,5-di0 -isopropylidene $-\alpha-\underline{\underline{D}}-\mathrm{gluc}$ furanose derivatives . . . . . . . . . . .

25. Chemical shifts ( $\tau$, p.p.m.) of 6-deoxy-l,2: $3,5-d i-0$-is opropylidene- $\alpha-D-g l u c$ ofuranose derivatives . . . . . . . . . . . . .

26. Determination of inososes with the Somogyi reagent. . . . . . . . . . . . . . . .

27. Magnetic parameters for the Cu(II) complexes of the Somogyi (A), Fehling (B), Reeves (C), and Benedict $(D)$ reagents at $77 \mathrm{~K} . . .$. .

28. Reagents used to detect hydrazine after generation from 2 on a t.l.c. plate. . 
ORGANIC CHEMISTRY SECTION: SUMMARY OF ACTIVITIES

JULY 1969 TO JUNE 1970

Edited by Robert Schaffer

This report of the Organic Chemistry Section of the National Bureau of Standard's Institute for Materials Research provides a summary of a year's scientific activity; as such, it covers both work that was completed and some that is still in progress. The development of standard Reference Materials (SRMS) and their certification constitute a significant output that we term the sample aspect of our programs. The research component appears in the form of journal publications.

About one-half of the report concerns SRMs. Spectrophotometric measurements of bilirubin and of "azobilirubin" in simple solvents and in serum or proteins are given much attention. D-Glucose for use as a clinical standard is examined by gas---liquid chromatography and differential scanning calorimetry to ascertain changes in the proportions of the anomeric forms of the sugar during these measurements. Additional compounds for development as SRMs are cortisol, 4-hyaroxy-3-methoxy-DL-mandelic acid (VMA), D-mannnitol, and the reduced form of $\beta$-NAD. Some properties thus far studied on commercially available specimens are reported.

In carbohydrate research programs, the purity of 1,2:4,5-di-o-isopropylidene-D-fructose and the importance of using it pure for conversion into pure D-psicose are described. The proton magnetic parameters of the unquestioned, skew conformation of 3-0-benzoyl-1,2,4-0-

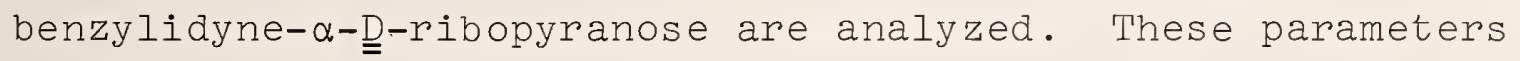
have major utility as a model for conformational analysis. Iterative analysis of p.m.r. spectra and the conformations of some D-glucose derivatives are described. Also, work is reported on cyclic polyhyäroxy compounds, including 
oxidation, electron spin resonance, and new derivatives.

Finally, a number of other topics are described:

iodination of $\beta$-diketones with periodic acid, and reactions of bilirubin, hemin, and related bile pigments; also, some work on the detection of polynitro aromatic compounds is given.

Key words: "Azobilirubin"; bilirubin; $\beta$-NAD; $\beta$-NADH; cholesterol; cortisol; D-mannitol; electron spin resonance; proton magnetic resonance; skew conformations; Standard Reference Materials; uric acid; VMA.

\section{CLINICAL STANDARDS}

In 1967, the Section undertook to develop the first of the Bureau's SRMs specifically directed to filling a need in laboratories doing clinical chemical analysis. Cholesterol (SRM 911) was issued that year, and since then the Section was responsible for the Urea (SRM 912), Uric Acid (SRM 913), and Creatinine (SRM 914) that have been issued. A few months ago, a tally of laboratories served with these SRMs numbered in excess of 700 ; many were outside the United States.

Two additional organic SRMs are about to be issued. These are SRM Bilirubin and SRM D-Glucose. Considerable effort was required to develop criteria for the Bilirubin SRM because of the difficulty of working with a material that is not stable at its melting point, nonvolatile, poorly soluble, and unstable. At this writing, the SRM itself is scheduled to be evaluated in July 1970, and therefore, data on it are not included in this report. We have been very much concerned with the methods by which the SRM bilirubin can be dissolved, and combined with serum proteins; for analytical use, it is required in that matrix. 
We report some of the results we have obtained by using such procedures.

Clinical laboratories perform D-glucose analyses by many methods-chemical as well as biochemical (i.e., with enzymes). To ensure that the reference material is compatible with all clinical techniques, the D-glucose used for the clinical SRM has been more thoroughly characterized than the Dextrose (SRM 4la) currently issued by NBS for the chemical reducing methods used widely in industrial carbohydrate technology .

Development of four new SRMs for clinical analysis has begun. These are Cortisol, 4-Hydroxy-3-methoxy-DLmandelic Acid (VMA), D-Mannitol, and the reduced form of $\beta$-Nicotinamide Adenine Dinucleotide ( $\beta-N A D H)$.

Some data collected on two of the SRMs previously issued, namely, Cholesterol and Uric acid, are reported. We have been reexamining the former of these from time to time since it was issued, in order to ascertain evidence of any change in its purity. The latter had not been reexamined by us since it was certified.

\section{A. Cholesterol. Post-issuance Studies}

In Technical Note 457, we reported analyses performed on the cholesterol SRM six months after issuance under a provisional certificate, and, in Technical Note 507 , further studies at two additional six-month intervals. The objective of this continuing study is the evaluation of the shelf-life of the standard, particularly as it is known that cholesterol may undergo decomposition. The material has now been tested again.

\section{Thin-layer Chromatography of Cholesterol Samples \\ (A. Cohen)}

Chromatography was performed on activated Silica Gel G plates with $1: 1(\mathrm{v} / \mathrm{v})$ ethyl acetate-heptane as described 
in Technical Note 457. Use of an atmosphere (in the chamber) that was not saturated with vapors of the solvents gave better resolution.

Samples of the bulk supply of cholesterol that had been stored in a freezer for 30 months showed no significant evidence of change, but samples that were sealed in amber bottles kept at room temperature exposed to laboratory fluorescent lighting for 22 months, and samples in amber bottles that were loosely capped to ensure exposure of the cholesterol to air for 22 months, were found to be increasingly less pure, respectively, than the freezer-stored supply. No attempt was made to assess these changes quantitatively.

2. Liebermann-Burchard Analysis

(A. Cohen)

Measurements made by our modification of the Liebermann-Burchard method for cholesterol (see Technical Note 457) showed no significant difference between samples of the freezer-stored cholesterol and those left exposed to air and light for nearly 2 years.

3. Differential Scanning Calorimetry (DSC) and Phasesolubility Analysis (PSA)

(R. F. Brady, Jr.)

DSC and PSA were applied to cholesterol samples as described in Technical Note 457 [1]. The purity values for the freezer-stored cholesterol were found to be 99.7 mole-percent by DSC, and 99.7 weight-percent by PSA. Samples kept exposed to air in amber containers at room temperature for six months were found to be 99.4 mole-percent pure by DSC and 99.4 weight-percent pure by PSA. 
B. Urea (Clinical). Post-issuance Studies

1. Determination of Purity of Urea by Differential Scanning Calorimetry

(B. Coxon)

By use of ultra-pure indium ( $2.5620 \mathrm{mg}$ ), a calibration factor of $0.02360 \mathrm{mcal} / \mathrm{unit}$ area was determined at the time of this study.

Portions of urea $(0.9485 \mathrm{mg}$ of sample 1 , and $1.0840 \mathrm{mg}$ of sample 4) were heated in sealed sample-pans from $401 \mathrm{~K}$ to $406 \mathrm{~K}$ at $0.625 \mathrm{~K} / \mathrm{min}$. The thermograms were divided into seven sections between $2402.5 \mathrm{~K}$ and $405.5 \mathrm{~K}$, and the area of each section was determined in duplicate by planimetry.

The values of purity obtained were 99.82 mole-percent for sample 1 , and 99.81 mole-percent for sample 4; these results are essentially unchanged from those determined on the same samples two years earlier.

2. Determination of Moisture Content of Urea by Karl Fischer Titration

\section{( J . H. Thomas)}

Portions of urea $(4.8388 \mathrm{~g}$ and $4.7266 \mathrm{~g}$ of sample $\mathrm{I}$ and $4.7196 \mathrm{~g}$ and $4.7252 \mathrm{~g}$ of sample 4) were dissolved in absolute methanol, and titrated with Karl Fischer reagent to an end point of $45 \mu \mathrm{A}$ on a Waco titration apparatus. The Karl Fischer apparatus was first calibrated with milligram quantities of water in the range of the moisture content of the urea sample. In this calibration, the weights of water used divided by the corresponding volumes (V) of Karl Fischer reagent required give a factor (F). The expression

$$
\frac{F \times V \times 100}{\text { weight of urea }}
$$


gives the \% of moisture in the sample. The percentages of moisture in the two samples were 0.050 and 0.047 ; and 0.048 and 0.050 , respectively.

3. Determination of Purity of Urea by Phasesolubility Analysis

(R. F. Brady, Jr.)

An analysis of the purity of a sample of urea was performed by using phase-solubility analysis. The solvent chosen was isopropyl alcohol, in which urea is soluble to the extent of $29.0 \mathrm{mg} / \mathrm{g}$ of solvent. For details of the technique, see reference [2]. The sample of urea was found to be $99.9 \%$ pure; the accuracy of the technique is considered to be $\pm 0.1 \%$. Within experimental error, this analysis is in excellent agreement with the analysis of purity by differential scanning calorimetry.

C. Bilirubin

(B. F. West and B. A. Johnson)

1. Standard Solutions of Bilirubin

Bilirubin in serum exists (a) as the free compound adsorbed on serum albumin, as, otherwise, free bilirubin would exhibit its high insolubility in aqueous media, and (b) in a soluble, conjugated form, e.g., as the derivative in which each of its carboxyl groups is esterified by $\mathrm{OH}-\mathrm{I}$ of $\underline{\underline{D}}$-glucuronic acid.

Although the development of standards for bilirubin conjugates is not now feasible because of difficulties in isolating and purifying such conjugates from natural sources or, alternatively, difficulties in their synthesis, bilirubin itself is available, and it is used for standardizing clinical assays. By modification of the conditions of coupling with diazotized sulfanilic acid, clinical chemical procedures afford the means for obtaining a 
separate estimate of (a) the proportion of the conjugated bilirubin, and (b) the sum of the conjugated and free bilirubin.

In 1962, a committee with representatives from the American Academy of Pediatrics, the College of American Pathologists, the American Association of Clinical Chemists, and the National Institutes of Health recommended a procedure for preparing solutions of bilirubin in serum for use in standardizing clinical assays [3]. In their words, "an accurately weighed quantity of an 'acceptable bilirubin' is dissolved completely and as quickly as possible (less than $5 \mathrm{~min}$ ) in subdued light at room temperature in $0.1 \mathrm{M}$ sodium carbonate solution, the quantity of the latter being selected to constitute 2 percent of the final volume of the prepared standard. [Occasionally, bilirubin preparations are encountered which are difficult to dissolve in the carbonate. When this occurs, $0.05 \mathrm{M}$ sodium hydroxide may replace the $0.1 \mathrm{M}$ sodium carbonate, provided the resultant solution is used within 5 min.] (These two bracketed sentences appear as a footnote.) The clear red solution is immediately diluted with an 'acceptable serum diluent' to a final volume selected to give a desired final concentration of not less than $5 \mathrm{mg} / 100 \mathrm{ml}$. An 'acceptable serum diluent,' to be used both in the preparation of this standard and in any subsequent dilutions for calibration purposes, is defined tentatively as pooled serum having an absorbance of less than 0.100 at $414 \mathrm{m \mu}$ and 0.040 at $460 \mathrm{m \mu}$ at a dilution of 1:25 in $0.85 \% \mathrm{NaCl} . "$ The recommended procedure (and the Committee's accompanying criteria for the bilirubin and the serum to be used for standards preparation) proved to be a milestone in upgrading clinical analysis for bilirubin.

As a careful reading of these direction might suggest, bilirubin is not readily dissolved within the recommended 
conditions, nor is it easy in subdued light to see whether all of a specimen has dissolved in the deep-red solution that is produced. Furthermore, once dissolved in alkali, bilirubin undergoes decomposition. The net effect is a great likelihood of a less-than-optimal preparation of the standard solution, particularly if the procedure is carried out only occasionally or by an analyst unaware of the many pitfalls.

Some further salient facets needing attention were added by Gambino [4]: "Several precautions must be observed when applying the Committee recommendations on a uniform bilirubin standard. The standard should be made with human serum or plasma that is less than 4 hours old and that has been protected from light and heat. Many samples of older, heated, or irradiated sera caused slight to severe suppression of azobilirubin color when tested by the Submitter in four variations of the Malloy-Evelyn method, the Powell-o'Hagan method, and the Jendrassik-Grof method. Michaelsson and Schellong also recommended fresh serum. The standard must be protected from light. The pH of the standard should be brought to 7.3-7.5. When bilirubin crystals are dissolved in sodium carbonate, there should not be any brown tint, and, when the same crystals are dissolved in chloroform, there should not be any brown tint. If $5 \mathrm{vol}$. of chloroform-bilirubin solution are washed with 1 vol. of $5 \%(\mathrm{w} / \mathrm{V})$ sodium bicarbonate, the bicarbonate wash should be free of dark brown pigment."

When our work on developing a bilirubin standard reference material led us to the preparation of bilirubinenriched sera, we recognized the desirability of finding an alternative means for effecting dissolution of the crystalline material_-one that would avoid the use of aqueous alkali. We attempted to do this with methyl sulfoxide ( $\operatorname{Me}_{2} \mathrm{SO}$ ) as solvent, and only later learned that 
work with this solvent had already been published by Gadd [5]. Billing, Halsam, and Wald [6] have further studied the use of this solvent. In their work, proportions of $\mathrm{Me}_{2} \mathrm{SO}$ in the serum are utilized that far exceed the 2 percent $(\mathrm{v} / \mathrm{v})$ restriction employed in the American Committee's method; also, they used frozen, pooled serum (not fresh serum) as the diluent. We describe some work along these lines.

A further modification of the dissolution procedure has been described by Dybkaer and Hertz [7], who used a $0.1 M$ solution of potassium cyanide in formamide to dissolve the bilirubin. In it, bilirubin is stable for several hours, and a remarkably high concentration of the pigment can be obtained. The solution, which they denoted as B-CN-F (bilirubin in potassium cyanide-formamide), is readily used to enrich fresh, pooled serum to as high as $25 \mathrm{mg} / 100 \mathrm{ml}$. Most interestingly, on fractionation by size-chromatography, the enriched serum (made to $\mathrm{pH} 9.5$ before adding the $\mathrm{B}-\mathrm{CN}-\mathrm{F}$ ) was found to have the bilirubin distributed only in the albumin fraction, as found in hyperbilirubinemic serum. Hence, their technique employs serum made alkaline to that $\mathrm{pH}$ prior to enrichment, and the enriched preparation is neutralized afterwards for storage. The authors reported the utility of such enriched sera as standards for the assay, by direct spectrophotometric measurement, of the free bilirubin of hyperbilirubinemic sera.

Without having in hand the bilirubin that would be certified as the Bureau's SRM, we undertook to examine bilirubin samples by utilizing the clinical-chemical techniques of bilirubin diazo-coupling, in the absence or presence of protein, and bilirubin spectrophotometry. By assuming that each bilirubin sample was pure, millimolar absorptivities were calculated, to provide a basis for comparison for each test and technique. As the bilirubin content of the sample examined is not accounted for in the 
calculation, these millimolar absorptivities have only relativistic value.

a. Some Estimates of the Millimolar Absorptivities of Bilirubin in Non-aqueous Solvents

Our customary procedure was to weigh (by microbalance) 3.5 to $7 \mathrm{mg}$ of bilirubin on a pan, and then wash the sample into a 0.5- to 1-liter volumetric flask by use of solvent. Thus, dilution was not normally employed to bring the solution to a concentration that would provide a reading of about 0.7 absorbance unit on our Cary 14 spectrophotometer. Dissolution in chloroform usually required brief warming at $\sim 70^{\circ} \mathrm{C}$ in a water bath, and this was performed under subdued, incandescent light. Volumes were adjusted at $20^{\circ} \mathrm{C}$, uut solutions were warmed to $25^{\circ} \mathrm{C}$ prior to recording of their spectra. Unless otherwise specified, the chloroform used was reagent grade that contains $0.75 \%$ of ethanol.

i. In Chloroform

Two portions of a bilirubin sample were weighed. One was heated in the dark at $117^{\circ} \mathrm{C} / 0.3$ torr in an Abderhalden apparatus for $10 \mathrm{~h}$. Then the two samples were dissolved in reagent chloroform. The heated sample showed $\varepsilon_{m M} 61.7$; tre unheated sample showed $\varepsilon_{\mathrm{m} M}$ 61.5. The similarity of values showed that the heat-treatment had little effect on the result; alternatively, the content of volatile material in this sample seemed to be low, and heating did not appear to "harm" the bilirubin.

Another sample of bilirubin was simultaneously examined in hydrocarbon-stabilized chloroform and in (our usual) ethanol-stabilized chloroform. The $\varepsilon_{m M}$ values obtained were 61.0 and 60.6, respectively. The difference, although small, appears to be significant; we have obtained other, similar data. 
In our extraction experiments, we had observed that, after extraction with water, chloroform solutions of bilirubin invariably show lower absorbances, and, to learn whether this was related to the selective removal of the ethanol from the chloroform, we measured the absorbance of bilirubin dissolved in chloroform that had been freed of ethanol by extracting it twice with water and then drying $\left(\mathrm{Na}_{2} \mathrm{SO}_{4}\right.$ ) and distilling. In the ethanol-free chloroform, we obtained an $\varepsilon_{m M} 58.7$ On addition of ethanol to this solution to a volume fraction of $0.002, \varepsilon_{m M}$ increased to 59.3 , and $\varepsilon_{m M}$ remained at 59.3 when the ethanol content of the solvent was raised to 0.4 and then to 1 percent. Hence, the composition of the chloroform used in obtaining the millimolar absorptivity of bilirubin must be carefully specified. The $\lambda_{\max }$ of these chloroform solutions was $453 \mathrm{~nm}$.

\section{ii. In Methyl Sulfoxide}

Bilirubin dissolves very readily in this solvent. A minute or so of immersion of the mixture in the water of an ultrasonic-cleaning bath ensures completion. Because of the costliness of the solvent, only 2 to $3 \mathrm{mg}$ of bilirubin was employed and the solutions were made to $250 \mathrm{ml}$. The values of $\varepsilon_{m M}$ are $\sim 64$, distinctly higher than found in chloroform; the $\lambda_{\max }$ is like that found in chloroform.

\section{iii. In Formamide Made $0.1 \mathrm{M}$ in Potassium Cyanide}

This solvent is prepared by dissolving an appropriate weight of the salt in formamide. Bilirubin solutions in this solvent were made to $\sim 7 \mathrm{mg} / \mathrm{l}$. As in methyl sulfoxide, dissolution is facile and is ensured by a brief ultrasonic treatment. The values of $\varepsilon_{\mathrm{m} M}$ in this solvent are $\sim 64$, and the $\lambda_{\max }$ is 453 , as in methyl sulfoxide and in chloroform. 
The Evelyn-.-Malloy procedure for total bilirubin in serum [8] was adapted to the examination of bilirubin in methyl sulfoxide alone (i.e., without any serum or protein present). We used a concentrated solution of bilirubin in $\mathrm{Me}_{2} \mathrm{SO}$ and, at first, diluted this with methanol, the diluent normally employed in the procedure with serum. However, there was wide scatter in replicate tests, and this seemed to be due to the turbidity that resulted from admixture with the methanol. The latter was therefore replaced by $\mathrm{Me}_{2} \mathrm{SO}$ as the diluent, and a great improvement in precision resulted.

In this modification, $110 \mathrm{mg}$ of bilirubin (carefully weighed) was dissolved in $25 \mathrm{ml}$ of $\mathrm{Me}_{2} \mathrm{SO}$, and $1.0-\mathrm{ml}$ aliquots were diluted to $50 \mathrm{ml}$ with $\mathrm{Me}_{2} \mathrm{SO}$. For testing, aliquots $(5 \mathrm{ml})$ of the latter solution were mixed with equal volumes of the diazo reagent. Three replicates on two dilutions of the same bilirubin-- Me ${ }_{2} \mathrm{SO}$ solution gave an average $\varepsilon_{m M}$ of 50.1 (range $49.3-50.7$ ).

Experiments were then conducted to determine whether there might be an optimal $\mathrm{pH}$ for the reaction in $\mathrm{Me}_{2} \mathrm{SO}$. The concentration of hydrochloric acid in the diazo reagent was therefore varied, and it was found that an increase in $\varepsilon_{m M}$ resulted from use of a lower proportion of hydrochloric acid. This change in $\varepsilon_{m M}$ was accompanied by a wavelength shift toward the red; the visible color of the diazotized mixture was pink. As use of less than $0.1 \mathrm{ml}$ of the concentrated acid, the condition giving the highest value of $\varepsilon_{m M}$, was deemed undesirable, further investigations of effects of $\mathrm{pH}$ were not pursued. Hence, the optimal pH was not ascertained. Data are given in table 1 . 
Table 1. Dependence of absorption on pH, for "azobilirubin" in methyl sulfoxide

Concentrated

hydrochloric

acid $(\mathrm{ml})^{\mathrm{a}}$
Final diazo mixture

$\mathrm{pH}$

$3 \cdot 31$

2.93

2.92

2.73

2.35

2. 27

2.13

2.09

1.98

1.93

1.80

1.92 $\lambda_{\max }$

$(\mathrm{nm})$

528

529

532

537

538

542

544

547

550

552

554

555 $\varepsilon_{m M}$

61.9

60.6

59.6

58.6

58.4

57.9

56.4

55.5

54.7

53.5

52.4

52.1

$\overline{a_{\text {Per }} 100} \mathrm{ml}$ of sulfanilic acid solution.

When two different stock solutions of bilirubin in $\mathrm{Me}_{2} \mathrm{SO}$ were diluted with additional $\mathrm{Me}_{2} \mathrm{SO}$ to provide concentrations from 14.5 to $3 \mu \mathrm{g} / \mathrm{ml}$, and these were tested with the diazo reagent of "optimal composition," a linear relationship was found for the absorbances measured at $528 \mathrm{~nm}$ at the concentrations tested.

From time to time over a period of two months, two commercial bilirubins were remeasured by this diazo procedure, each test involving weighing, dissolution, and diazo-coupling. One material showed, in 5 tests, an average $\varepsilon_{m M}$ of 62.1 , with results ranging from 61.2 to 62.9; the other material showed, in 4 tests, an average $\varepsilon_{m M}$ of 62.5 and a range of 61.8 to 62.9 . 
A sample of a commercial, lyophilized, bilirubincontrol material (bilirubin on bovine albumin) was examined by the $\mathrm{Me}_{2} \mathrm{SO}$-modified Malloy-- Evelyn procedure. The sample was reconstituted according to the manufacturer's directions, and then further diluted with $\mathrm{Me}_{2} \mathrm{SO}$ to a suitable concentration. The $\lambda_{\max }$ for the diazo-coupled product was the same as we had found for bilirubin diazo-coupled in the absence of the protein (i.e., $528 \mathrm{~nm}$ ); however, the $\varepsilon_{\mathrm{m} M}$ was found to be 50.6 based on the concentration stated for the samples. An $\varepsilon_{m M}$ lower than $\sim 62$ was to be expected, in keeping with the well-known effect of protein on the millimolar absorptivity of "azobilirubin."

3. Millimolar Absorptivities for Bilirubin Diazo-coupled by the Jendrassik-Grof Procedure

In this method, the coupling reaction of bilirubin with diazotized sulfanilic acid at $\mathrm{pH} \sim 5.5$ produces a red solution. Addition of alkali before the absorbance is measured brings the $\mathrm{pH}$ to $\mathbf{1 3 . 4}$ and changes the color to green. Variations of the basic method that enhance its usefulness have been reported in the literature.

As our interest was the utilization of bilirubin in preparing standard solutions for use by clinical laboratories to calibrate their analyses, we determined values of $\varepsilon_{m M}$ for the bilirubin in these solutions, as well as for the diazo product obtained.

The excellent, bilirubin-dissolving capacity of formamide made $0.1 M$ in potassium cyanide was used by Dybkaer and Hertz [7] for enriching serum pools prepared from freshly collected blood. We attempted, at first, to perform the diazo-coupling of the B-CN-F (see p. 9) solution in the absence of added protein, by the Jendrassik-Grof procedure as described by Gambino [4], with the aim, again, of obtaining values of $\varepsilon_{\mathrm{m} M}$ uncomplicated by the presence 14 
of protein; however, this procedure did not seem to be usable without protein present, and further effort along this line was abandoned. In its place, we worked with B-CN-F and its enrichment of human sera.

A number of separate preparations of $B-C N-F$ at a bilirubin concentration of $\sim 7 \mathrm{mg} / \mathrm{l}$ were scanned by spectrophotometry, and were found, at $\lambda_{\max } 453 \mathrm{~nm}$, to give $\varepsilon_{m M} \sim 64$. These absorption spectra were otherwise very similar in appearance to that of solutions of bilirubin in chloroform.

Dybkaer and Hertz specified that fresh, pooled, human serum be used as the matrix for enrichments. As adequate supplies of fresh, human serum are not always available, it was of interest to us to examine the applicability of frozen, pooled serum, especially as this was available to us for use. A rather cloudy serum was used in the first of our enrichments with B-CN-F. The serum had been made alkaline (to $\mathrm{pH}$ \%9.5), and was then enriched. We followed Dybkaer and Hertz's use of tetraborate buffer as diluent to afford direct spectrophotometric measurement of the bilirubin, and found, with non-enriched serum (diluted with buffer) as the blank, that $\varepsilon_{m M}$ of the bilirubin corresponded to 50 for eight entirely separate enrichments. But, on diazo-coupling, the $\varepsilon_{\mathrm{m} M}$ values obtained ranged widely, from 77 to 90, values that individually are much higher than might have been expected. With use of the same serum (not made basic beforehand), the values of $\varepsilon_{m M}$ for "diazobilirubin" were still in the $80^{\prime} \mathrm{s}$. We think that these very high $\varepsilon_{\mathrm{m} M}$ values may be due as much to turbidity as to the use of "old" serum.

Further work with B-CN-F enrichments was next conducted on fresh serum that we obtained (by way of the Medical Office at NBS) $4-5 \mathrm{~h}$ after the blood had been drawn. After adjustment of the $\mathrm{pH}$ to $\sim 9.5$, the serum was treated with 
$\mathrm{B}-\mathrm{CN}-\mathrm{F}$, to provide a concentration of bilirubin of $\sim 20$ $\mathrm{mg} / 100 \mathrm{ml}$ of serum; then, the serum was neutralized with an amount of acid equivalent to the alkali initially used. Aliquots of this serum were separately frozen at $-28^{\circ} \mathrm{C}$; aliquots of non-enriched serum were frozen as well. Thawed aliquots were diluted (1:10) with $0.85 \%$ saline for (a) direct spectrophotometry, with tetraborate buffer used to effect a further 1:10 or 2:5 dilution, and water used as the blank; and (b) diazo-coupling following Dybkaer and Hertz, employing the Michaelsson modification of the Jendrassik-. Grof procedure.

Four different serum-pools were used. Pool 1 was enriched with one preparation of $\mathrm{B}-\mathrm{CN}-\mathrm{F}$, and aliquots were studied over a period of several days. Pool 2 was enriched with 3 different B-CN-F solutions; similarly, pools 3 and 4 were each divided into three separate portions, and each was enriched with a new B-CN-F concentrate. Each enrichment with B-CN-F provided a concentration of bilirubin of $\sim 20 \mathrm{mg} / 100 \mathrm{ml}$ of serum. The results obtained by study of each aliquot for bilirubin and "diazobilirubin" millimolar absorptivities are shown in table 2 .

We were also interested in knowing how purified, humanserum albumin might behave as a matrix for enrichment with B-CN-F. For this purpose, commercial, lyophilized, human-serum albumin was dissolved in $0.85 \%(\mathrm{w} / \mathrm{V})$ saline, to provide a $2.5 \%(\mathrm{w} / \mathrm{v})$ solution of the protein. Portions of this solution were enriched with B-CN-F, either (a) with no special pretreatment, (b) after being brought to $\mathrm{pH} 9.5$. and then neutralized, or (c) enriched as in (b), but not subsequently neutralized. However, it was found that, independent of their mode of preparation, aliquots obtained from these enrichments were very unstable. As an alternative, the albumin solution was made up in a phosphate buffer at 


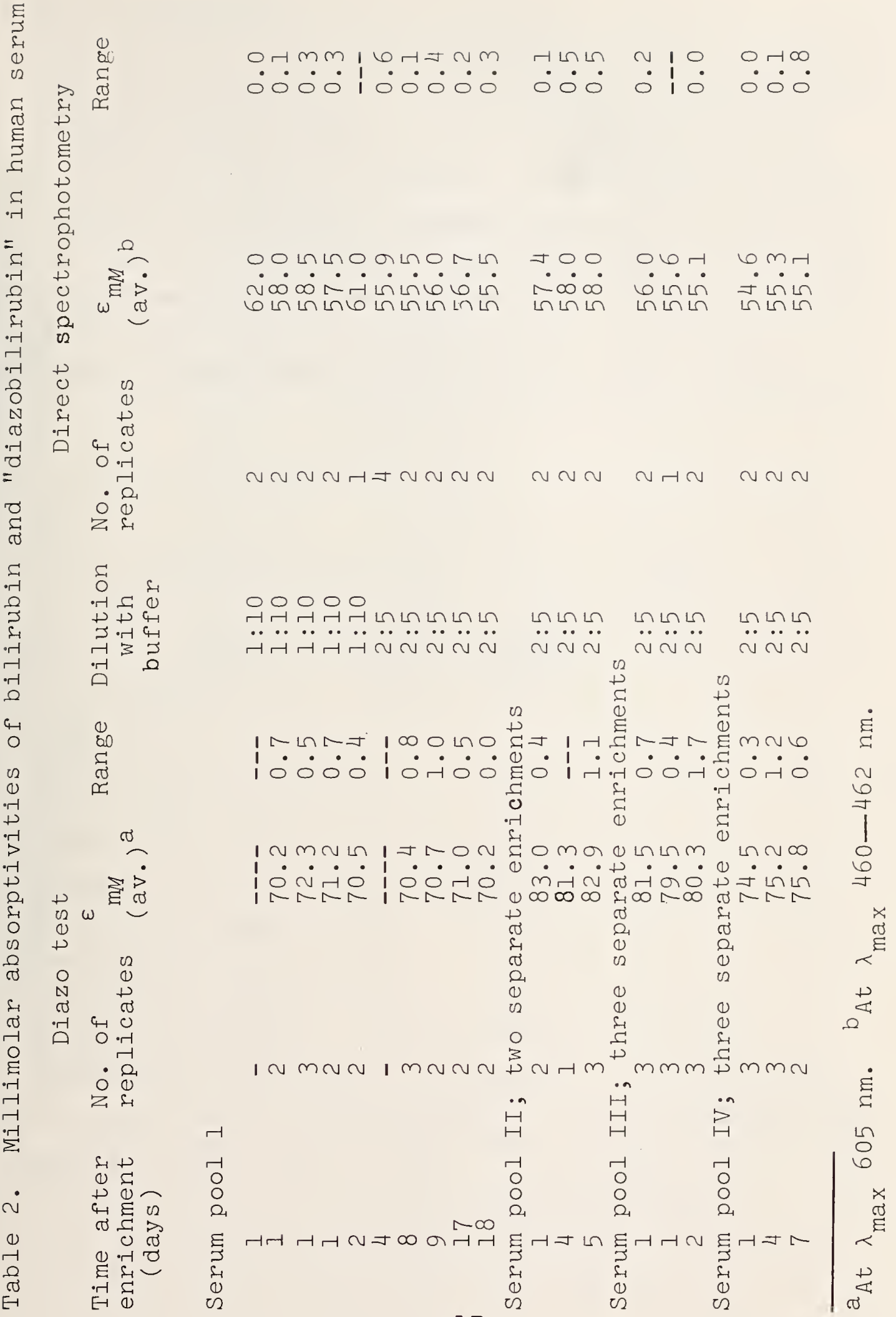


pH 7.4 (following a suggestion by Dr. A. Hainline), and enriched as in (a) utilizing B-CN-F and, for comparison, with solutions of bilirubin in $0.05 \mathrm{M}$ sodium hydroxide.

Table 3 provides an overall summary of the results from these enrichments. The values of $\varepsilon_{m M}$ given for each enrichment are averages obtained from the denoted number of replicates by each procedure. Enrichments 1--5 with B-CN-F provided values of $\varepsilon_{m M}$ that were much less reproducible than those of 6--10 (by sodium hydroxide). The ranges of $\varepsilon_{\mathrm{mM}}$ do not reveal the distinct trend to diminish with time that was found in the B-CN-F enrichments.

Table 3. Millimolar absorptivity of bilirubin in human-serum albumin

Enrichment Base ${ }^{a}$

\begin{tabular}{cccccc} 
Time & No. of & \multicolumn{4}{c}{$\varepsilon_{\mathrm{mM}}$} \\
$\begin{array}{c}\text { Studied } \\
\text { (days) }\end{array}$ & $\begin{array}{c}\text { replic- } \\
\text { ates }\end{array}$ & \multicolumn{2}{c}{ Direct } & \multicolumn{2}{c}{ Diazo } \\
11 & 8 & 55.4 & 1.2 & 71.2 & 1.2 \\
10 & 6 & 50.9 & 1.7 & 68.4 & 2.0 \\
6 & 10 & 53.4 & 1.3 & ---- & --- \\
6 & 6 & 52.4 & 0.9 & 66.9 & 2.1 \\
12 & 8 & 52.2 & 1.8 & 67.3 & 2.1 \\
8 & 10 & 55.5 & 0.4 & ---- & --- \\
12 & 8 & 55.6 & 0.9 & 70.6 & 4.2 \\
8 & 8 & 55.2 & 1.2 & 68.9 & 1.0 \\
8 & 4 & 57.9 & 1.4 & 68.6 & 2.0 \\
8 & 4 & 55.4 & 0.8 & 67.8 & 1.2
\end{tabular}

$\mathrm{a}_{\mathrm{A},} 0.1 M \mathrm{KCN}-\mathrm{F} ; \mathrm{B}, 0.05 M$ sodium hydroxide. ${ }^{\mathrm{b}}$ At $\lambda_{\max } \sim 467$ $\mathrm{nm} . \mathrm{C}_{\text {At }} \lambda_{\max } 600 \mathrm{~nm}$. 
D. $\quad$ D-Glucose (Clinical)

Analyses for $\stackrel{D}{\underline{D}}$-glucose in blood constitute an important diagnostic test, and thus, in the near future, NBS will issue standard samples of $\underline{\underline{D}}$-glucose for clinical use. Dextrose, issued by NBS as Standard Reference Material 4la, is the standard for chemical reducing methods of sugar analysis. The clinical standard, for which more extensive investigations of the physical properties of the $\underline{\underline{D}}$-glucose, its quantitative purity, and impurities have been made, is intended for enzymic and other clinical methods. Preliminary analyses were described in a previous report [9].

1. Analysis of $\stackrel{\text { D-Glucose by Gas-liquid Chromatography }}{ }$

(B. Coxon and J. H. Thomas)

a. Crystalline Material

In order that a minimum proportion of the $\alpha-\underline{\underline{D}}-\mathrm{glucose}$ would be anomerized to $\beta-\underline{\underline{D}-g l u c o s e, ~ p o r t i o n s ~(~} 5 \mathrm{mg}$ ) of samples $2 \mathrm{~B}$ and $4 \mathrm{~B}$ were per(trimethylsilyl)ated by use of $1 \mathrm{ml}$ of Tri Sil $\mathrm{Z}$ at $0^{\circ} \mathrm{C}$ for $10 \mathrm{~min}$. The anomers were separated on a glass column ( 6 ft. $x$ 0.25 in. O.D.); the solid support was Chromosorb WAWS (80--100 mesh) with a liquid phase of $3 \%$ of SE-30. The column was operated isothermally at $180{ }^{\circ} \mathrm{C}$, and the separated components were detected with a flame-ionization detector operated at $220{ }^{\circ} \mathrm{C}$. The sample size was $1 \mu l$.

The areas of the peaks due to $\alpha$ and $\beta$ anomers were obtained by triangulation. The percentages of $\alpha$ and $\beta$ anomers were then calculated from the total areas, with the assumption that anomeric trimethylsilyl derivatives have the same response factor.

The percentages of $\beta-D-g l u c o s e$ found, for four determinations on each sample, were: for $2 \mathrm{~B}, 0.43 ; 0.40$; 0.42 ; and 0.50 ; for $4 \mathrm{~B}, 0.53 ; 0.60 ; 0.52$; and 0.53 . 


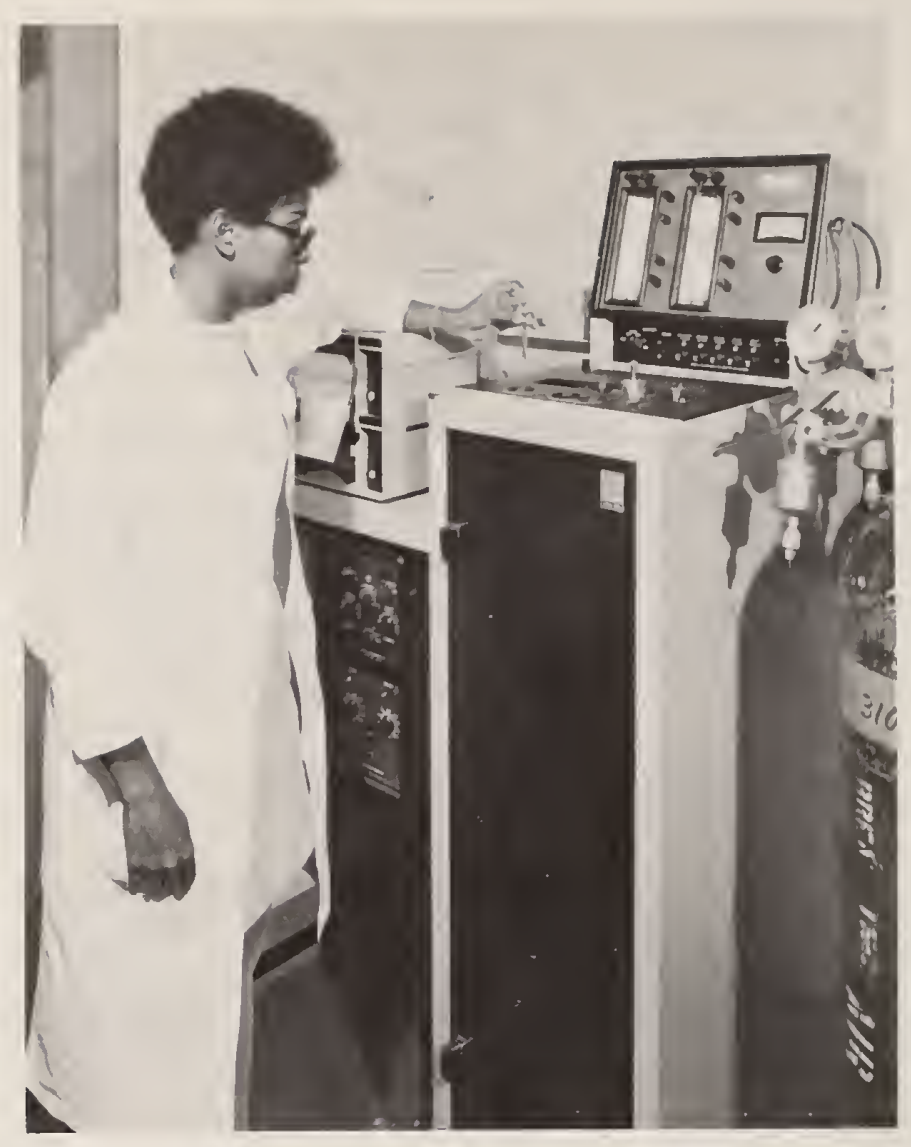

Figure 1. Determination of the purity of a volatile compound by gas-liquid chromatography.

b. Melted Samples of $\underline{\underline{D}-G l u c o s e}$

In order to assess the significance of the determination of the purity of D-glucose by differential scanning calorimetry, samples of $\underline{\underline{D}-g l u c o s e}$ were heated under nitrogen in the calorimeter and were analyzed by $\mathrm{g} .1 . \mathrm{c}$. of the trimethylsilyl ethers at various stages of the melting process. 
Samples of D-glucose ( $23 \mathrm{mg}$ ) in open aluminum pans were flushed six times with nitrogen, and were heated from $380 \mathrm{~K}$ to $414 \mathrm{~K}$ at $80 \mathrm{~K} / \mathrm{min}$. Heating of each sample was then continued; one sample was heated to a maximum temperature $\left(\mathrm{T}_{\max }\right)$ of $418 \mathrm{~K}$, corresponding to a small amount of premelting, a second sample was heated to $420 \mathrm{~K}$, corresponding to the maximum rate of heat input on the DSC curve (melting $~ 60 \%$ complete), and a third sample was melted completely $\left(T_{\max } 421 \mathrm{~K}\right)$.

At $T_{\max }$, the aluminum sample-pan was rapidly removed from the calorimeter, and was allowed to cool to room temperature. The melted $\stackrel{\equiv}{-g l u c o s e}$ was then trimethylsilylated by stirring the sample pan and contents with Tri Sil $\mathrm{Z}$ ( $1 \mathrm{ml}$ ) at $0^{\circ} \mathrm{C}$ for $\mathrm{l} \mathrm{h}$. The resulting solutions were then analyzed by g.l.c. as described in 1 (a). The results are shown in table 4 .

Table 4. Isomerization of D-glucose during melting to $T_{\max }$

$\begin{array}{lccc}\mathrm{T}_{(\mathrm{K})} & \alpha \underset{(\%)}{\operatorname{anomer}} & \beta \underset{(\%)}{\operatorname{anomer}} & \text { Other components } \\ & & & \\ 298^{\mathrm{b}} & 99.5 & 0.5 & 0 \\ 418 & 97.8 & 2.2 & 0 \\ 420 & 55.2 & 43.5 & 1.3 \\ 421 & 45.3 & 52.3 & 2.4\end{array}$

a Probably furanoid or acyclic forms.
bnmelted. 
The fact that isomerization reactions proceed during the differential scanning calorimetry of D-glucose shows that the results for purity obtained by this technique should be treated with some caution. However, in spite of this, surprisingly good agreement was obtained between the proportions of $\alpha$-D-glucose measured by DSC and by g.l.c.

It is easily shown that heats of isomerization of the order of 278--.500 cal/mole* have little effect on the mole-\% purity measured, as these heats of isomerization are small compared with the heat of fusion of D-glucose (see table 5).

2. Apparent Purity of D-Glucose by Differential Scanning Calorimetry

(B. Coxon and R. F. Brady, Jr.)

By slight variations in procedure with DSC, different values can be obtained for the mole-percent purity of the D-glucose.

\section{a. Initial Procedure}

Calibration of a Perkin-Elmer Model DSC-IB instrument against ultrapure indium ( $2.562 \mathrm{mg}$ ) gave a calibration factor of $0.02658 \mathrm{mcal} /$ unit area of recording chart.

Sample weights were determined on a Cahn electrobalance which was calibrated against a standard 5-mg weight before each weighing. Portions of $\underline{\underline{D}}$-glucose $(2.8895 \mathrm{mg}$ of sample 4B, and $3.3720 \mathrm{mg}$ of sample $2 \mathrm{~B}$ ) in open sample-pans were evacuated and flushed with nitrogen six times and were heated from $380 \mathrm{~K}$ to $414 \mathrm{~K}$ at $80 \mathrm{~K} / \mathrm{min}$, and then from $414 \mathrm{~K}$

* Literature values [10] of 278 and $299 \mathrm{cal} / \mathrm{mole}$ for $\alpha$-D-glucose in aqueous solution at 25 and $35{ }^{\circ} \mathrm{C}$, respectively. If it is assumed that the heat of isomerization is proportional to temperature, a value of $536 \mathrm{cal} / \mathrm{mole}$ can be estimated for $421 \mathrm{~K}$ (the mp of $\alpha-\underline{\underline{D}-g l u c o s e) .}$ 
at $0.625 \mathrm{~K} / \mathrm{min}$. The segment of each DSC thermogram between 418 and $424 \mathrm{~K}$ was analyzed by planimetry, duplicate measurements being made on each of eight sectors under the curve to assure reproducibility. Purity values thus obtained were 98.61 and 98.58 mole-percent with portions

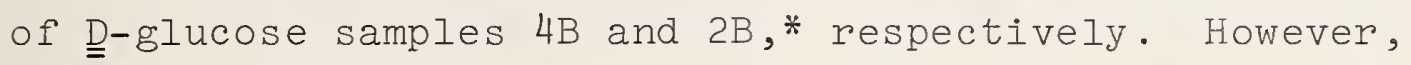
the presence of a proportion of $\beta$ anomer (see section 1 ) and the moisture content must be borne in mind.

\section{b. Effect of Duration of Heating on the Apparent Purity}

The calculated purity of $\stackrel{D}{=}$-glucose was found to be dependent on the duration of the slower heating prior to melting. Portions of D-glucose were heated in open sample-pans under nitrogen from $298 \mathrm{~K}$ to temperature $\mathrm{T}$ at $80 \mathrm{~K} / \mathrm{min}$, and then from $\mathrm{T}$ at $0.625 \mathrm{~K} / \mathrm{min}$ up to the melting point $\left(\mathrm{T}_{\mathrm{m}}\right)$. Higher calculated values of mole-percent purity were obtained when the duration of heating before melting was lessened, presumably because of decrease in the extent of anomerization or dehydration (or both) that would lower the purity of the sample. The results are summarized in table 5 .

Table 5. Effect of duration of heating at $0.625 \mathrm{~K} / \mathrm{min}$ on the apparent purity of D-glucose (clinical) as measured by differential scanning calorimetry

\begin{tabular}{cccccc}
$\begin{array}{c}\mathrm{T} \\
(\mathrm{K})\end{array}$ & $\begin{array}{c}\mathrm{T}_{\mathrm{m}} \\
(\mathrm{K})\end{array}$ & $\begin{array}{c}\text { Duration of } \\
\text { slow heating } \\
(\mathrm{min})\end{array}$ & \multicolumn{4}{c}{$\Delta_{F}$} & $\begin{array}{c}\text { Purity } \\
(\mathrm{mole}-\%)\end{array}$ \\
411 & 420.7 & 15.5 & 8280 & 34,700 & 98.75 \\
414 & 422.7 & 13.9 & 7860 & 32,900 & 99.03 \\
417 & 422.5 & 8.8 & 8300 & 34,700 & 99.40 \\
417 & 422.0 & 8.0 & 8210 & 34,400 & 99.41 \\
418 & 422.7 & 7.5 & 8100 & 33,900 & 99.47 \\
420 & 423.8 & 6.1 & 8100 & 33,900 & 99.43
\end{tabular}

* Determined on material as received from the manufacturer. 
3. Determination of Moisture Content of D-Glucose Samples

( $J$. H. Thomas)

Karl Fischer (K.F.) titrations of samples $4 \mathrm{~B}$ and $2 \mathrm{~B}$ of $D$-glucose were made by the standard method, except that Karl Fischer diluent was used instead of methanol. The moisture content was then cross-checked by another direct method, namely, the near-infrared (NIR) spectrophotometric method that is specific for water [1]], for which, in the present analysis, a cell having a 5-cm path-length was used, because of the low content of water. The percentages of water found are given in table 6 .

Table 6. Percent of water in samples of D-glucose Sample number

Water

$(\%)$

$4 \mathrm{~B}$

by K.F. titration

by NIR
0.055
0.055
0.051
0.057
0.055

$2 B$
0.056
0.052
0.053
0.060
0.055

E. Cortisol

(R. F. Brady, Jr.)

Cortisol ( $\underset{\sim}{1}$ ) is one of several hormones secreted by the

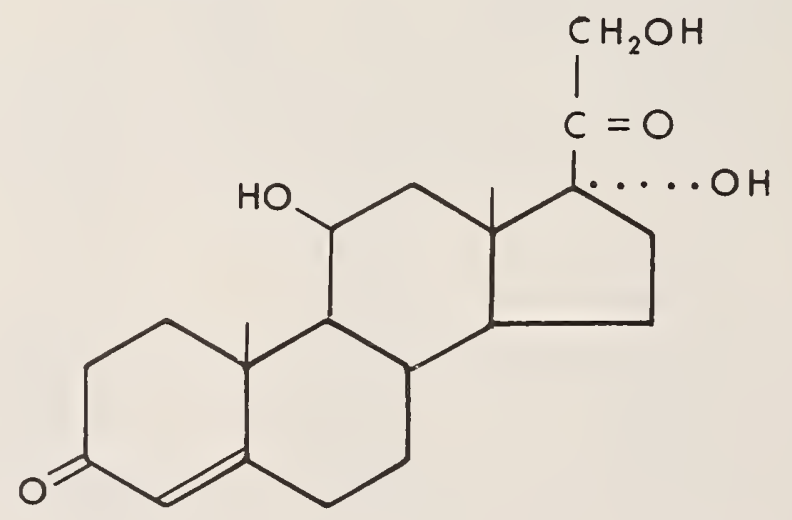


adrenal cortex. It is essential to the normal functioning of the human body, and many pathological conditions result from abnormalities in the amount of cortisol produced. Such malfunctions as Addison's disease are engendered by cortisol deficiency, whereas excessive amounts of this hormone may result in such equally serious disorders as Cushing's syndrome.

The evaluation of adrenocortical activity is a critical step in the diagnosis of these diseases, but, because of the variety of adrenocorticosteroids and their various reduction and degradation products, many difficulties are encountered in the determination of these compounds in plasma and urine.

A pure, crystalline cortisol is being developed as a Standard Reference Material for use as a clinical standard for assaying cortisol in body fluids.

\section{Physical Properties of Some Cortisol Samples}

\section{a. Melting Point}

Melting-point determinations in open Pyrex capillary tubes were made on four commercial samples of cortisol. Each tube was immersed in a bath preheated to $200{ }^{\circ} \mathrm{C}$, and was then heated at a rate of $0.5^{\circ} \mathrm{C} / \mathrm{min}$ until melting was complete. Their melting ranges are recorded in table 7 . The melting point of cortisol is given in the literature [12] as "217.-.220 with some decomposition."

\section{b. Optical Rotation}

Approximately $60 \mathrm{mg}$ of cortisol from each of the commercial sources was accurately weighed (to $10 \mu g$ ) into a 10.00-ml volumetric flask, and dissolved in sufficient absolute ethanol to make $10.00 \mathrm{ml}$ of solution. The samples appeared to differ in ease of dissolution. The optical rotation at $589 \mathrm{~nm}$ of each solution in a l-dm tube was measured by using a Perkin---Elmer Model 141 photoelectric 
polarimeter, and the rotations are given in table 7 . The optical rotation of cortisol is given in the literature [12] as $[\alpha]_{D}^{22}+167^{\circ}$ (c l, absolute ethanol).

\section{c. Elemental Analysis}

Results of elemental analyses of the four commercial samples of cortisol are given in table 7 . The values calculated for $\mathrm{C}_{21} \mathrm{H}_{30} \mathrm{O}_{5}$ are: $\mathrm{C}, 69.6 ; \mathrm{H}, 8.3 \%$.

Table 7. Some physical properties of samples of cortisol

$\begin{array}{lccccc}\begin{array}{l}\text { Manufacturer's } \\ \text { lot number }\end{array} & \begin{array}{c}\mathrm{m} \\ \left({ }^{\circ} \mathrm{C}\right)\end{array} & \begin{array}{c}{[\alpha]_{\mathrm{D}}^{22}} \\ (\text { degrees })\end{array} & \begin{array}{c}\text { Analys } \mathrm{C} \\ \% \mathrm{H}\end{array} & \mathrm{T}_{\mathrm{m}} \\ 52580 & 219-222 & +167.1^{\mathrm{a}} & 69.5 & 8.4 & 221 \\ 58 \mathrm{~B}-2240 & 212-216 & +166.8^{\mathrm{b}} & 69.6 & 8.3 & 211 \\ 2167 & 216-220 & +166.4^{\mathrm{a}} & 69.8 & 8.4 & 223 \\ \mathrm{U} 4174 & 220-224 & +164.9^{\mathrm{c}} & 69.3 & 8.3 & 218\end{array}$

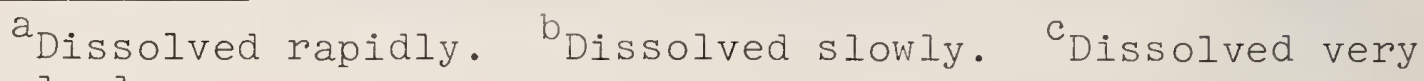
slowly.

2. Determination of Purity by Differential Scanning Calorimetry

Each sample of cortisol was examined by using a Perkin-. Elmer Model DSC-IB Differential Scanning Calorimeter. A weighed quantity of cortisol in an open aluminum dish was placed in the sample cell of the calorimeter, and the dish was covered with a hemispherical aluminum dome. A similarly covered, empty aluminum dish was placed in the reference cell. With the calorimeter cover locked in place, the sample chamber was flushed with a gentle stream of dry nitrogen for $3 \mathrm{~min}$ in order to remove oxygen. The calorimeter temperature was then raised from $298 \mathrm{~K}$ to about $5 \mathrm{~K}$ below the melting point, at a rate of $80 \mathrm{~K} / \mathrm{min}$, 
and then at a rate of $0.625 \mathrm{~K} / \mathrm{min}$, until melting was complete. The procedure of operating under a nitrogen atmosphere was used with the cortisol because the decomposition that attends the melting of cholesterol while it is exposed to air had thereby been prevented, and by employing this technique, satisfactory DSC analyses of the purity of cholesterol had, in fact, been obtained [1]. However, with cortisol, "decomposition" apparently continues to take place during melting, even under the foregoing conditions. Thus, the endotherms obtained continued to show extremely broad "melting" ranges and low peak-amplitudes; also, the temperature of maximum peak-amplitude $\left(T_{m}\right)$ was different for each sample. Hence, differential scanning calorimetry is not applicable for determining the purity of cortisol because of the instability of this compound on heating.

\section{Spectrometric Properties}

a. Infrared Spectrum

Commercial samples of cortisol in a potassium bromide pellet show a well-defined i.r. spectrum having major bands at 3440 (OH stretch), 3015, 2980, 2950, 2925, 2895 (all CH stretches), 1720 (C-20 carbonyl stretch), 1645 (C-3 carbonyl stretch), 1610 ( $C=C$ stretch), 1455, 1435, $1420,1395,1350,1325,1290,1280,1240,1210,1140,1125$ $1105,1070,1060,1040,1015,950,930,910,875,790,755$, and $710 \mathrm{~cm}^{-1}$.

Infrared spectra were recorded with a Perkin-Elmer Model 257 grating infrared spectrophotometer. The spectra of the four commercial samples were identical with the published spectrum [13]; no impurities were evident from inspection of any of these spectra. 
Each sample of cortisol ( $60 \mathrm{mg}$ ) was dissolved in 0.4 $\mathrm{ml}$ of methyl sulfoxide- $\underline{d}_{6}$ containing $2 \%$ of tetramethylsilane as the internal standard. Spectra were obtained by using a Varian A-60 spectrometer operating at $37^{\circ} \mathrm{C}$. The n.m.r. spectrum of cortisol contains a "methylene envelope" from \$0.6 to 2.8, upon which are superimposed two singlets for the protons on $\mathrm{C}-18(\delta 0.66)$ and $\mathrm{C}-19(\delta 1.40)$. A singlet at $\delta 3.38$ may be assigned to the proton of the hydroxyl group on $\mathrm{C}-17$, and a broad peak (having some fine structure) at $\delta 4.1--5.0$ may be assigned to the protons of the two other hydroxyl groups, on $\mathrm{C}-11$ and $\mathrm{C}-2 \mathrm{l}$. Two additional singlets, at $\delta 5.12$ and 5.57 , may be assigned to the protons on $\mathrm{C}-2 \mathrm{l}$ and $\mathrm{C}-4$, respectively. The spectra of the four commercial samples were superposable, and none showed any evidence of impurity.

\section{Thin-layer Chromatography of Cortisol}

Thin-layer chromatography was performed with Silica Gel $G$, activated at $110^{\circ} \mathrm{C}$, as the adsorbent, coated on glass plates $(10 \times 20 \mathrm{~cm})$; the depth of the adsorbent layer was $250 \mu \mathrm{m}$. Chloroform-methanol (97:3, v/v), hexane---ethyl acetate ( $I: I, V / v)$, and benzene--isopropyl alcohol ( $4: I$, $\mathrm{v} / \mathrm{v}$ ) were the developing solvents. Samples in acetone (2 $\mu \mathrm{g} / \mu \mathrm{l}$ ) were applied in small spots by using a Hamilton syringe. After development, plates were examined under ultraviolet light, and in no instance was any zone made visible. Plates were then sprayed with methanol-concentrated sulfuric acid $(5: 1, \mathrm{~V} / \mathrm{V})$ and heated at $110{ }^{\circ} \mathrm{C}$ for $10-15$ min to develop the spots. The results are summarized in table 8 . 
Table 8. T.1.c. data on commercial samples of cortisol

Manufacturer's Developing lot number solventa

$R_{F}$

52580

$58 \mathrm{~B}-2240$

2167

$\mathrm{U} 4174$

52580

B

$58 \mathrm{~B}-2240$

2167

$\mathrm{U} 4174$

52580

$58 \mathrm{~B}-2240$

2167

$\mathrm{U} 4174$
A

0.09

0.19

0.09

0.03

0.09

0.19

0.09

0.20

0.08

0.11

0.08

0.08

0.08

C

0.59

0.59

0.59

0.59
Identification

cortisol

cortisone

cortisol

unknown

cortisol

cortisone

cortisol

cortisone

cortisol

cortisone

cortisol

cortisol

cortisol

cortisol

cortisol

cortisol

cortisol

a A, Chloroform-methanol $(97: 3 \mathrm{v} / \mathrm{v})$; B, hexane-ethyl acetate $(I: I, v / v)$; C, benzene-isopropyl alcohol $(4: I, v / v)$.

The identification of one of the minor spots as cortisone

(2) was made by comparison with authentic cortisone in the

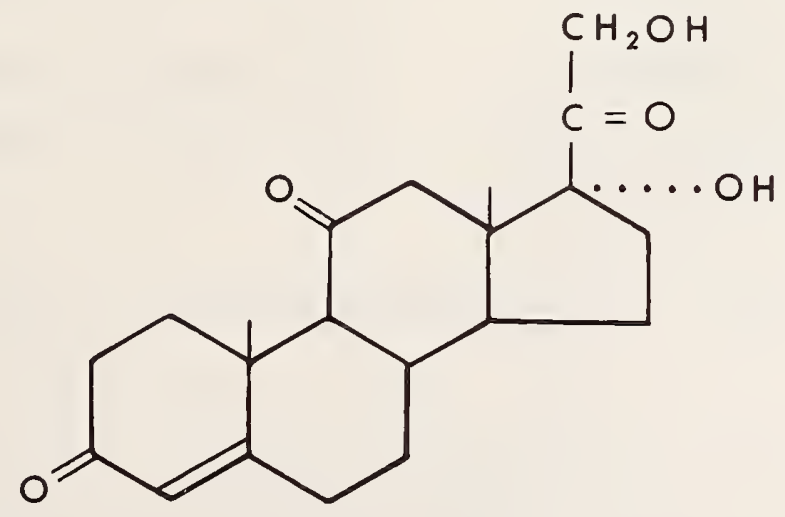


three developing solvents employed. The $R_{F}$ values were: in chloroform--methanol (97:3, v/v), 0.19; in hexane-ethyl acetate $(I: I, V / V), 0.11$; and in benzene-isopropyl alcohol $(4: 1, v / v), 0.59$.

F. 4-Hydroxy-3-methoxy-DL-mandelic Acid (VMA)

(R. S. Tipson, A. J. Fatiadi, and R. F. Brady, Jr.) 4-Hydroxy-3-methoxy-D-mandelic acid ( $\underset{\sim}{\text { D }})$ (vanil-,
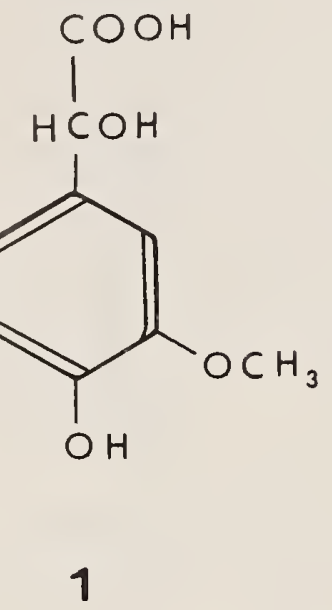

vanillyl-, or vanillin-mandelic acid, VMA) is a constituent of normal and pathological human urine, as it is the major urinary metabolite of epinephrine and norepinephrine. Consequently, it is important that a well-characterized VMA reference standard be made available for use in clinical laboratories. For the assays used by clinical chemists, the DL form gives the same responses as the $\underline{\underline{D}}$ form, and so we have studied samples of the racemate.

1. Melting Point of Commercial Samples of VMA (A. J. Fatiadi)

Melting-point determinations were made, on samples [seven commercial, and one that we had purified by successive recrystallization from l:l (v/v) benzene- 
acetonitrile and then from acetonitrile] of DL-VMA, in Pyrex capillary tubes with the contents exposed to the air. These tubes were placed in a preheated bath (at $125^{\circ} \mathrm{C}$ ) and heated at a rate of $1{ }^{\circ} \mathrm{C} / \mathrm{min}$ to the temperature of melting (or decomposition). The melting range was read from a mercury thermometer and is reported, without correction, in table 9.

Table 9. Melting points ${ }^{a}$ of samples of VMA

Sample lot no. Melting point ${ }^{b}$

$\left({ }^{\circ} \mathrm{C}\right)$

recrystallized

$134-135$

86085

$133-134$

none

$132-134$

100-0220

$131.5-132.5$

5113

$131-132$

V-1451

$130-132$

1449

$130-131$

900796

129-131

atiterature [14] mp, 134--135 ${ }^{\circ} \mathrm{C}$ (with decomposition). $\mathrm{b}_{\text {With }}$ decomposition; uncorrected.

2. Thin-layer Chromatography of Commercial Samples of VMA

(A. J. Fatiadi)

Thin-layer chromatography was performed on layers ( $250 \mu \mathrm{m}$ thick) of adsorbent coated on glass plates ( 5 x 20 or $10 \times 20 \mathrm{~cm}$ ). The adsorbents tested were (1) cellulose powder activated at $105^{\circ} \mathrm{C}$ for $10 \mathrm{~min}$, and (2) Silica Gel G, not activated. The developing solvents employed with adsorbent 1 were: butyl alcohol saturated with 3 Mydrochloric acid (solvent A), 20:20:I (v/v) amyl alcohol-formic acid-water (solvent $\mathrm{B}$ ), and $13: 7$ (v/v) propyl alcohol-water (solvent $C$ ). For adsorbent 2 , the solvent used was $4: 1: 1$ (v/v) chloroform-acetic acid-water 
(solvent D). For detection of spots with adsorbents 1 or 2, a spray of diazonium solution was used; this consisted of a $1: 1: 2(\mathrm{v} / \mathrm{v})$ mixture of (a) a solution of $0.1 \mathrm{~g}$ of p-nitroaniline in $2 \mathrm{ml}$ of $3 \mathrm{M}$ hydrochloric acid, (b) a solution of $0.2 \mathrm{~g}$ of sodium nitrite in $100 \mathrm{ml}$ of water, and (c) a solution of $10 \mathrm{~g}$ of potassium carbonate in $100 \mathrm{ml}$ of water. The plate was developed, sprayed, and dried at $60{ }^{\circ} \mathrm{C}$ for $30 \mathrm{~min}$; fluorescent spots were located under u.v. light $(254 \mathrm{~nm})$. This spray detected $\sim 2.5 \mathrm{\mu g}$ of VMA.

For plates of adsorbent 2, a spray consisting of $3 \mathrm{M}$ sulfuric acid in methanol could also be used. After the plate had been sprayed, it was heated at $110{ }^{\circ} \mathrm{C}$ for $3 \mathrm{~min}$, giving nonfluorescent, dark-brown spots that changed to purple. By use of this spray, $\sim 4 \mu \mathrm{g}$ of VMA could be detected.

The following approximate $R_{F}$ values were observed for VMA: adsorbent $1,0.90$ (solvent A), 0.71 (B), 0.61 (C); adsorbent $2,0.80$ (A) and 0.23 (D). The results obtained for commercial samples of VMA examined with adsorbent 1 , solvent $A$, and the diazonium spray are given in table 10 .

\section{Spectrometric Properties}

(A. J. Fatiadi)

a. Infrared Spectrum

Infrared spectra were recorded with a Perkin-Elmer Model 257 grating infrared spectrophotometer. The purified sample of VIA (in a potassium bromide pellet) gave a well-defined i.r. spectrum having major bands at $3350(\mathrm{OH})$, 2980 and $2940(\mathrm{C}-\mathrm{H}$ stretch), 1870,1748 and 1715 ( $\mathrm{C}=0$ stretch), 1610, 1520, 1440, 1385, 1330, 1275 and 1240 (=C-0-C, aromatic ether), 1200, 1160, 1140, 1070, 1040, $870,835,785,740$, and $710 \mathrm{~cm}^{-1}$ (benzene ring vibration). The spectra of the seven commercial samples were superposable.

b. Nuclear Magnetic Resonance Spectra

A sample ( $70 \mathrm{mg}$ ) of purified VMA was dissolved in 
Table 10. T.1.c. data on commercial samples of VMA

\begin{tabular}{|c|c|c|}
\hline $\begin{array}{l}\text { Manufacturer's } \\
\text { lot number }\end{array}$ & ${ }_{F}^{R}$ values & Identific \\
\hline 86085 & $\begin{array}{l}0.90 \\
0.04 \text { (trace) }\end{array}$ & $\begin{array}{l}\text { VMA } \\
\text { U }\end{array}$ \\
\hline None & $\begin{array}{l}0.92 \\
0.31\end{array}$ & $\begin{array}{l}\text { VMA } \\
\mathrm{U}\end{array}$ \\
\hline $100-0220$ & $\begin{array}{l}0.89 \\
0.34 \\
0.24 \\
0.06\end{array}$ & \}$_{\mathrm{U}}^{\mathrm{VMA}}$ \\
\hline 5113 & $\begin{array}{l}0.91 \\
0.62 \\
0.28 \\
0.03\end{array}$ & \}$^{\mathrm{VMA}}$ \\
\hline 900796 & $\begin{array}{l}0.91 \\
0.62 \\
0.35 \\
0.02\end{array}$ & \}$_{\mathrm{U}}^{\text {VMA }}$ \\
\hline$V-1451$ & $\begin{array}{l}0.88 \\
0.32 \\
0.18 \\
0.12\end{array}$ & \}$^{\mathrm{VMA}}$ \\
\hline 1449 & $\begin{array}{l}0.90 \\
0.59 \\
0.32 \\
0.05\end{array}$ & \}$^{\text {VMA }}$ \\
\hline
\end{tabular}

$0.5 \mathrm{ml}$ of methyl sulfoxide- $\underline{\mathrm{d}}_{6}$ containing $2 \%$ of tetramethylsilane as the internal standard. Spectra were obtained by using a Varian A-60 spectrometer operating at $32-35{ }^{\circ} \mathrm{C}$. The n.m.r. spectrum showed signals for a singlet at $\delta 3.70\left(-\mathrm{OCH}_{3}\right.$ group); a singlet at $\delta 4.95$ (presumably due to $-\mathrm{C}-\mathrm{H}$, not exchangeable with $\left.\mathrm{D}_{2} \mathrm{O}\right)$; a multiplet centered at $\delta 6.8$ (aromatic protons), and a broad peak at $\delta 8.65$ that, on treatment with a drop of pyridine- $\underline{d}_{5}$ changed to a sharp singlet at $\delta 8.73$. The spectrum does not show clearly the phenolic hydroxyl proton, and the chemical shifts of other protons in the spectra depend very much on the nature of the solvent used. 
c. Mass-spectrometric Examination of Commercial Samples of VMA

\author{
(A. J. Fatiadi)
}

Mass spectra of seven commercial samples of VMA were analyzed for purity at a sample temperature of $\sim 140{ }^{\circ} \mathrm{C}$ with a Bell and Howell Consolidated Double-Focusing Mass Spectrometer Model 21-491. The spectra were measured, and were first examined for gross differences.

A calculation of the approximate purity was made by summing the ion current obviously arising from compounds other than the VIIA. Table 11 lists the samples in order of decreasing purity. The numerical value is a rough approximation of the impurity reiative to the content of the major component.

Table 11. Approximate percent of impurity in commercial samples of VIIA as indicated by mass spectrometry

Sample lot no.

none

$100-0220$

5113

86085

900796

$\mathrm{V}-1451$

1449
Total impurity, \%

$$
\begin{aligned}
& \sim 0.01 \\
& \sim 0.01 \\
& \sim 0.01 \\
& \sim 0.02 \\
& \sim_{0.08} \\
& \sim_{0.20} \\
& \sim_{0.25}
\end{aligned}
$$

On the basis of these results, the last 3 samples were not examined further. More-exact calculations of the contribution of impurities to the spectra of the first 4 samples and a recalculation of the order of purity were made. The numerical values shown in table 12 are the sums of ion currents due to readily identified ions not probable in the spectrum of VMA. The values may be compared to a value of unity for the molecule ion current of VMA; 
consequently, the recalculation showed the purity, in decreasing order, as given in table 12 .

Table 12. Re-estimation of purity of commercial samples of VMA by mass spectrometry

Sample lot no. 86085

none

$100-0220$

5113
Total impurity, \%

0.028

0.028

0.034

0.059

In addition, two commercial samples of VMA were heated for $4 \mathrm{~h}$ at 110 or $140^{\circ} \mathrm{C}$, and their mass spectra were compared with that of unheated material. The spectra of the first two samples, heated at $140{ }^{\circ} \mathrm{C}$, were found to contain a considerable proportion of a compound tentatively identified as vanillin. The proportion of the molecule ion of vanillin (from an authentic sample) was found to be 6 percent.

The spectrum of sample 100-0220 (heated at $100{ }^{\circ} \mathrm{C} / 1$ torr for $4 \mathrm{~h}$ ) was compared with that of unheated sample. Again, a large proportion of vanillin was found. The proportion of the molecule ion peak of VMA to the molecule ion peak of vanillin was found to be $25 \%$.

In the spectra of both mixtures, in addition to vanillin, small proportions of heavier ions were found in the vicinity of mass 300. The proportion of the mixture represented by these heavier ions (occurrence of decarboxylation of VMA at these temperatures is obvious) is probably less than $1 \%$ of the total. The mass spectra were measured by E. E. Hughes of the Microchemical Analysis Section, most of whose comments are provided. 
Table 13. Relative retention times and percentages of components of samples of per(trimethylsilyl)ated VMA and related compounds

Sample

lot no.

Major component Approximate Approximate retention time (min)

$\%$

16.6

16.9

900796

86085

None

$100-0220$

$V-1451$

$17 \cdot 5$

$17 \cdot 3$

99.0

99.1

$17 \cdot 1$

5113

1449

$17 \cdot 3$

99.7

$99 \cdot 3$

99.2

16.8

17.5

99.0

98.8

98.5

Vanillin

$7 \cdot 0$

Vanillic acid 10.0
Impurity $^{a}$ Approximate Approximate retention time (min)
$\%$

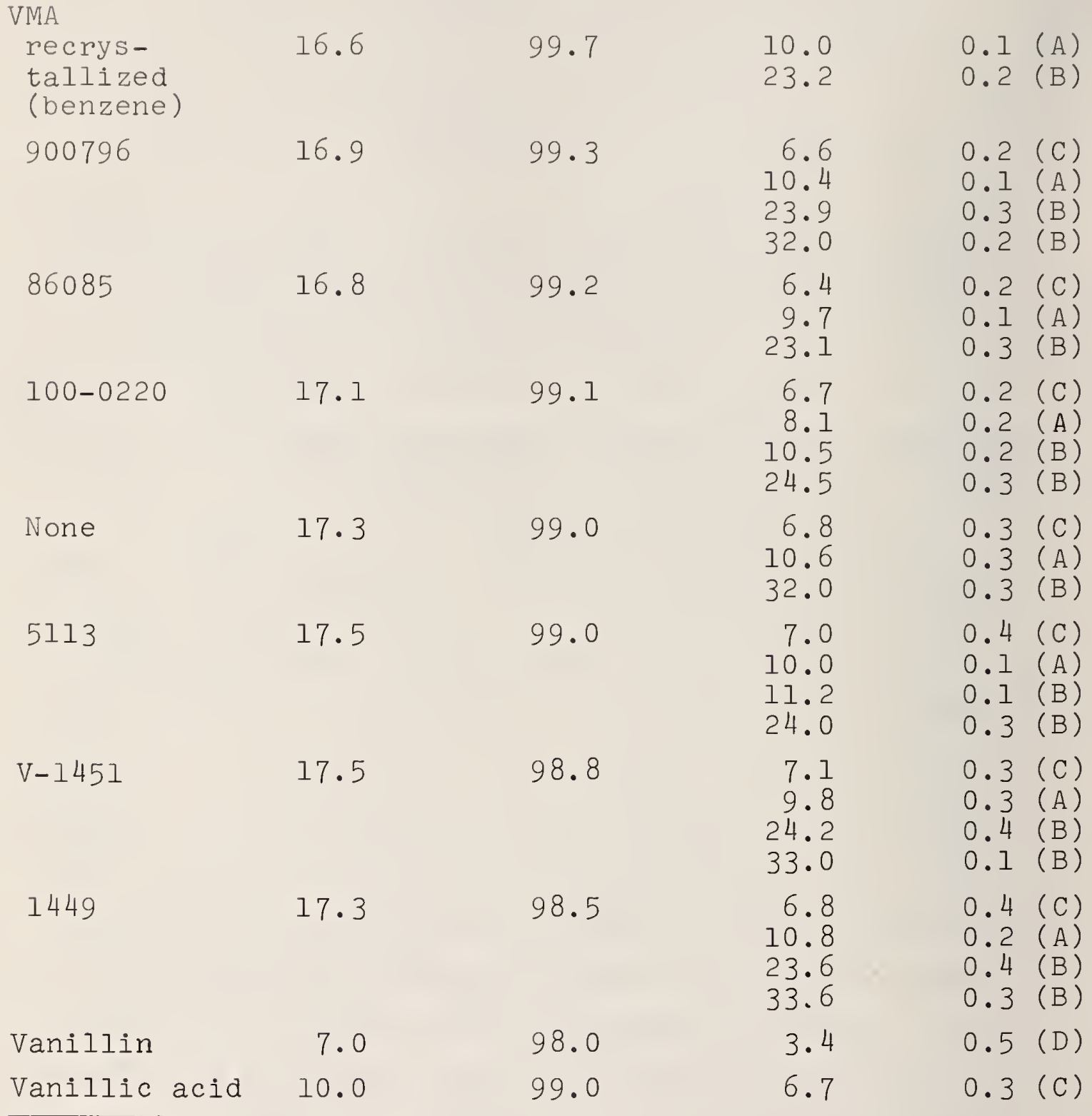

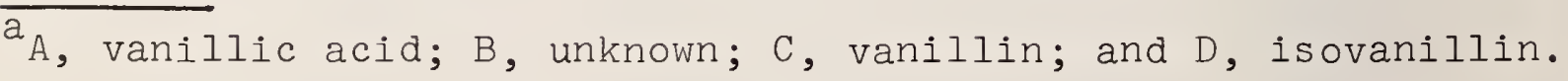


4. Examination by Gas--liquid Chromatography (G.I.c.) of the Per(trimethylsilyl) Derivatives of Samples of VMA

(A. J. Fatiadi)

Samples of VMA (seven commercial and one that we had purified) were individually examined by per(trimethylsilyl)ation, followed by g.l.c. Separation was achieved on a glass column ( $6 \mathrm{ft} . \mathrm{x} 2 \mathrm{~mm}$ i.d.) of Chromosorb Q (60--80 mesh) with a liquid phase of $3 \%$ of OV-17. The column was operated isothermally at $150{ }^{\circ} \mathrm{C}$, and the separated components were detected with a flameionization detector operated at $200{ }^{\circ} \mathrm{C}$; the temperature at the injection port was $180^{\circ} \mathrm{C}$. The sample size was $\sim 10 \mu \mathrm{g}$.

The samples were per(trimethylsilyl)ated by use of Tri Sil Z. Vanillin and vanillic acid were used as reference compounds. The results are summarized in table 13.

\section{Determination of Purity of VMA by Differential Scanning Calorimetry}

(R. F. Brady, Jr.)

Seven commercial samples of VMA were analyzed for purity by differential scanning calorimetry. The technique employed was similar to that described earlier [1] for the analysis of cholesterol by DSC. The results are summarized in table 14 .

Table 14. Analysis of purity of VMA by differential scanning calorimetry

Manufacturer's $\mathrm{m} \mathrm{p} \quad \Delta H_{F}$ lot number

$(\mathrm{K})$ 86085

None

5113

$100-0220$

$\mathrm{V}-1451$

1449

900798
399.1

399.2

399.7

400.2

398.6

399.8

a. $\mathrm{cal} / \mathrm{mol} \mathrm{J} / \mathrm{mol}$

$8270 \quad 34,620$

$8180 \quad 34,240$

$8000 \quad 33,490$

$8470 \quad 35,460$

$8270 \quad 34,620$

760531,830
Purity (mol \%)

99.2

99.1

99.0

98.9

98.6

97.7

\footnotetext{
$a_{\text {No }}$ distinct melting point.
} 
Fats, which occur as part of the lipid content of serum, are composed of a variety of fatty acid residues that are esterified to the glycerol moiety common to these compounds. Saponification liberates the glycerol, and it can be assayed by periodate cleavage of its vicinal carbon atoms, with the liberation of formaldehyde. The latter is estimated from the color produced by reaction with chromotropic acid. Only the primary carbon atoms are converted into formaldehyde; the secondary carbon atom provides formic acid, and chromotropic acid is unaffected by this acid. As a standard for fats by use of this test, glycerol would be very unsatisfactory. On the other hand, D-mannitol is a non-hygroscopic solid that can be purified to a high degree and can serve in place of the glycerol, because it affords the same number of formaldehyde molecules per molecule of it oxidized. Its higher molecular weight is advantageous for use as the standard.

Samples of D-mannitol were obtained from four different suppliers and are designated as samples 1, 2, 3, and 4.

\section{Thin-layer Chromatography (T.I.c.) of D-Mannitol}

T.l.c. was conducted on $20-\mathrm{cm}$ glass plates coated with Silica Gel G. The samples were applied to the plates as $0.5-$ or $1-\mu l$ aliquots of $10 \%$ solutions in water, corresponding to applied amounts of 0.05 and $0.1 \mathrm{mg}$, respectively. It was found that separation of D-mannitol from D-glucitol* is difficult to achieve, and so the use

*D-Glucitol is a likely impurity in preparations of D-mannitol that have been produced commercially by electrolytic reduction of $\underline{\underline{D}}$-fructose. 
of plates of Silica Gel G impregnated with various inorganic complexing reagents was investigated. In these studies, the unactivated layer of silica gel was sprayed with the solution of complexing reagent and then activated at $125{ }^{\circ} \mathrm{C}$ for $10 \mathrm{~min}$, before application of the sample, except that, for acidified ammonium molybdate, ammonium molybdate solution was sprayed on the plate, which was then activated, cooled, and sprayed with 5\% sulfuric acid. After air-drying of the plate, the samples were applied, and t.l.c. was performed immediately. The results are given in table 15.

Although small differences $(<0.1)$ of the $R_{F}$ values

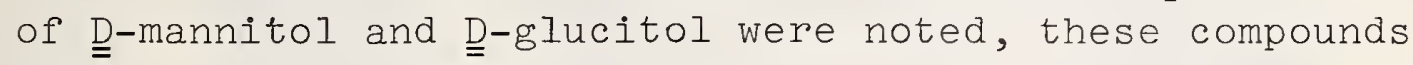
have not been separated completely by any of the systems thus far investigated. However, several of the methods afforded separations of impurities in the D-glucitol sample.

Table 15. Thin-layer chromatography of D-mannitol
Impregnation
Solvent ${ }^{a}$
$R_{F}$ value
reagent
None
A
0.4
Saturated boric
A
0.3
acid solution
B
0.3
Saturated
sodium tetraborate
solution
C
0.1
None
C
0.5
Ammonium
molybdate-
sulfuric acid
C
0.3

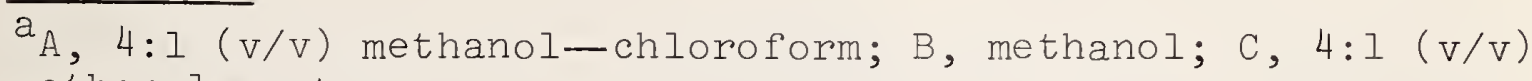
methanol-water.

\section{Paper Chromatography of D-Mannitol}

Aliquots $(5 \mu l)$ of $10 \%$ solutions of samples $1-4$ of D-mannitol (and of D-glucitol) in water were applied to Whatman filter paper No. 1, and paper chromatography was 
performed by the descending method with $8: 2: 1$ ( $\mathrm{v} / \mathrm{v}$ ) ethyl acetate-pyridine-water ( $0.5 \mathrm{mg}$ of each alditol). The chromatograms were sprayed with sodium metaperiodate solution ( $20 \mathrm{mM})$, followed after $2 \mathrm{~min}$ by silver nitrate solution (5\%). All of the samples of D-mannitol (Man) showed a trace of impurity having $R_{\text {Man }} 0.14$. Under these conditions, D-glucitol has $R_{\text {Man }} 1.12$, but this value is not sufficiently different from that of D-mannitol (1.0) to permit the detection of small amounts of D-glucitol in large applications of D-mannitol. The sample of D-glucitol was found to contain significant proportions of two impurities having $R_{\text {Man }} 0.22$ and 0.03 .

\section{Optical Rotation of Samples of D-Mannitol}

Ammonium molybdate solution was prepared by dilution of $50.000 \mathrm{~g}$ of the crystalline tetrahydrate to $1 \mathrm{dm}^{3}$ with distilled water. Each sample of D-mannitol ( 0.08 or $\sim 0.04 \mathrm{~g}$, accurately weighed) was dissolved in an aliquot $(20.0 \mathrm{ml})$ of ammonium molybdate solution. The resulting solution was then diluted to $25.0 \mathrm{ml}$ with $0.5 \mathrm{M}$ sulfuric acid.

Measurements were made either at $20{ }^{\circ} \mathrm{C}$, by use of a thermostated, l-dm cell in a Perkin-Elmer Model 141 polarimeter, or at $\sim 24{ }^{\circ} \mathrm{C}$ in a non-thermostated, 0.I-dm cell in a Cary Model 60 spectropolarimeter. With either of these instruments, measurements at wavelengths below $370 \mathrm{~nm}$ were not possible, owing to the very strong absorption of the acidified ammonium molybdate solution. Indeed, an ultraviolet absorption spectrum (recorded by A. Cohen) of a solution prepared by 1,000-fold dilution of acidified, stock, ammonium molybdate solution with water showed an absorption maximum at $214 \mathrm{~nm}$ (absorbance 21.45 ).

Solutions of D-mannitol in acidified ammonium molybdate solution are unstable, and, during storage at room temperature for $16 \mathrm{~h}$, deposit colorless crystals. On further storage, the supernatant liquid becomes bright blue. 40 
values of the specific rotation, mainly for the concentration range of $1.60-1.65$, are given in table 16.

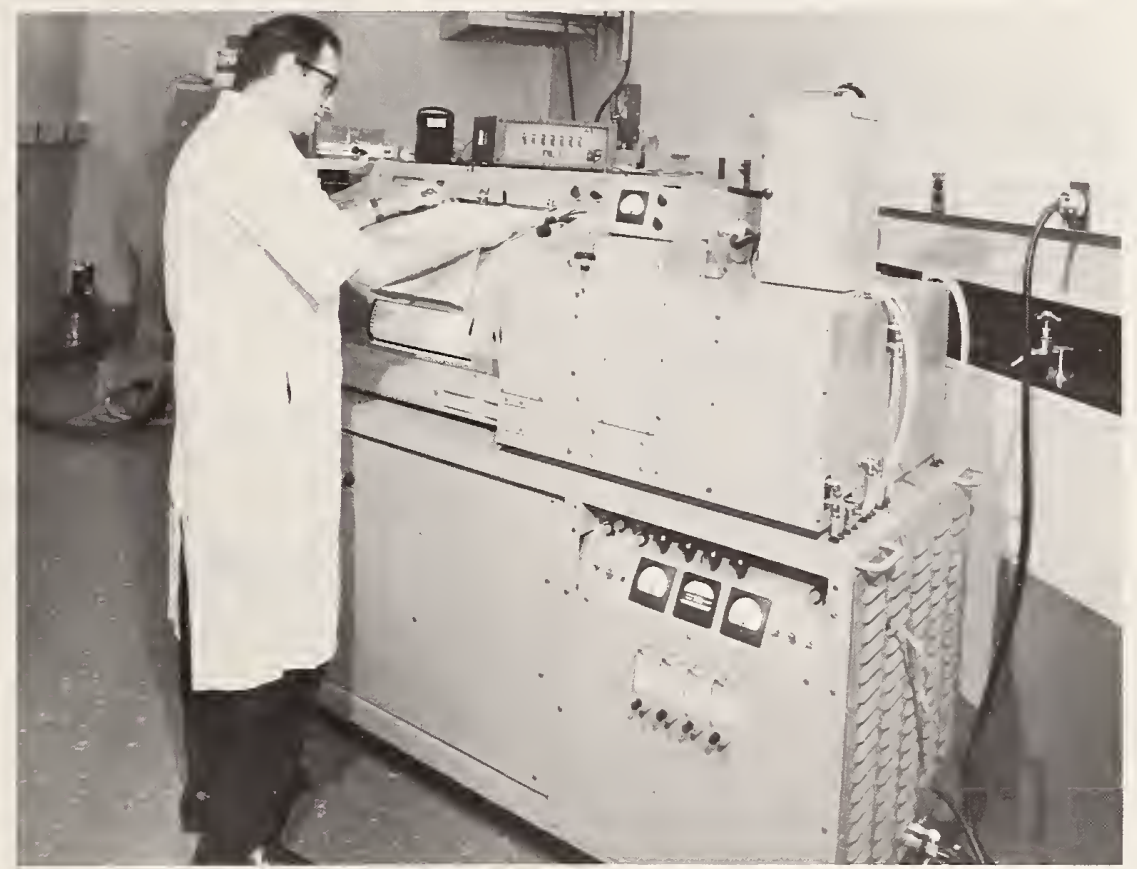

Figure 2. Recording an optical rotatory dispersion spectrum.

Table 16. Specific rotations, at various wavelengths, of solutions of commercial samples of D-mannitol in acidified ammonium molybdate solution

Wavelength

$(\lambda)$, in $\mathrm{nm}$ $[\alpha]_{\lambda}^{20}$, degrees, for samples $1-4$ 1 2 3 4 $589(\mathrm{Na}-\mathrm{D})+139.0^{\mathrm{a}}(141.9)^{\mathrm{b}}+138.6^{\mathrm{a}}+138.9^{\mathrm{a}}(141)^{\mathrm{c}}+138.9^{\mathrm{a}}$ 578 $145.1(144.4)^{\mathrm{b}}$ 145.5 $145.2(147)^{\mathrm{C}}$ 145.4

546 $166.1(167.3)^{\mathrm{b}}$ 166.0 $166.1(169)^{\mathrm{c}}$ 166.0 436 $297.3(299.8)^{\mathrm{b}}$ 297.3 $296.7(303)^{\mathrm{c}} \quad 297.2$

$\overline{a_{\text {Literature }}}$ value $[15],[\alpha]_{\mathrm{D}}^{20}+139.2^{\circ}$ (c 1.60). $\mathrm{b}_{\text {The values }}$ in parentheses were measured on a less concentrated solution (c 0.32 ); literature $[15]$ values, $[\alpha]_{D}^{20}+140.3$ and $+141^{\circ}$.

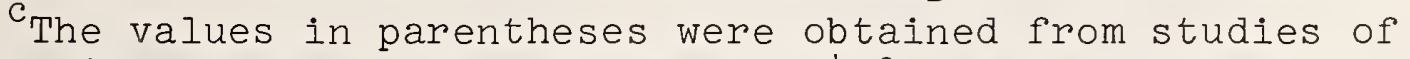
optical rotatory dispersion at $\sim 24{ }^{\circ} \mathrm{C}$, and are based on measurements of recorder chart-paper $(<7 \mathrm{~cm})$ with a precision of $\pm 0.2 \mathrm{~mm}$. 
H. B-Nicotinamide Adenine Dinucleotide ( $B$-NAD) and its 1,4Reduced Form ( $\beta-\mathrm{NADH})$

(R. S. Tipson, A. Cohen, and A. J. Fatiadi)

Among the organic compounds now being studied for

development as Standard Reference Materials for use in

clinical chemical analysis is $\beta-N A D H ~(2)$, the 1,4 -reduced form of $\beta-N A D(\underset{\sim}{I})$. Although $\beta-N A D$ was discovered in 1906, and

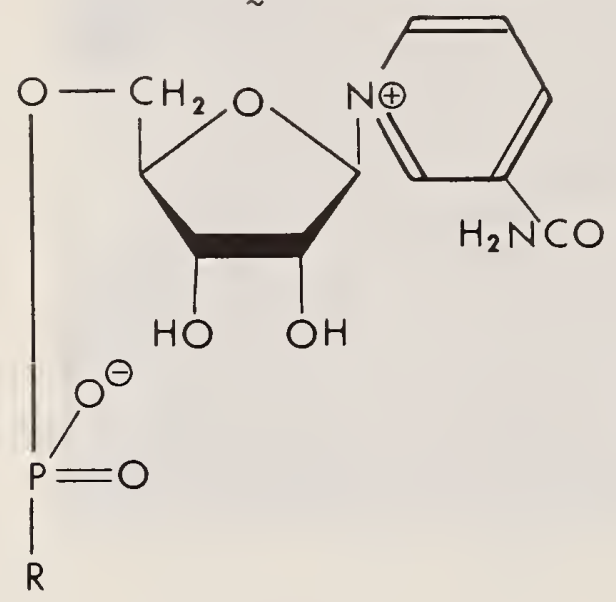

1

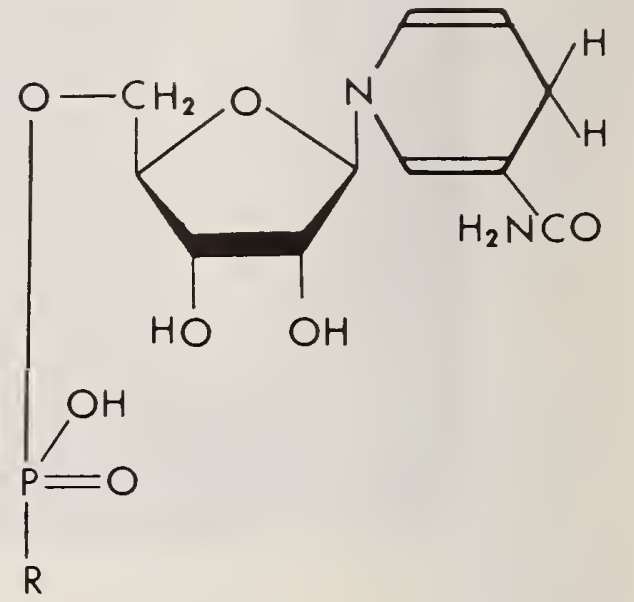

2

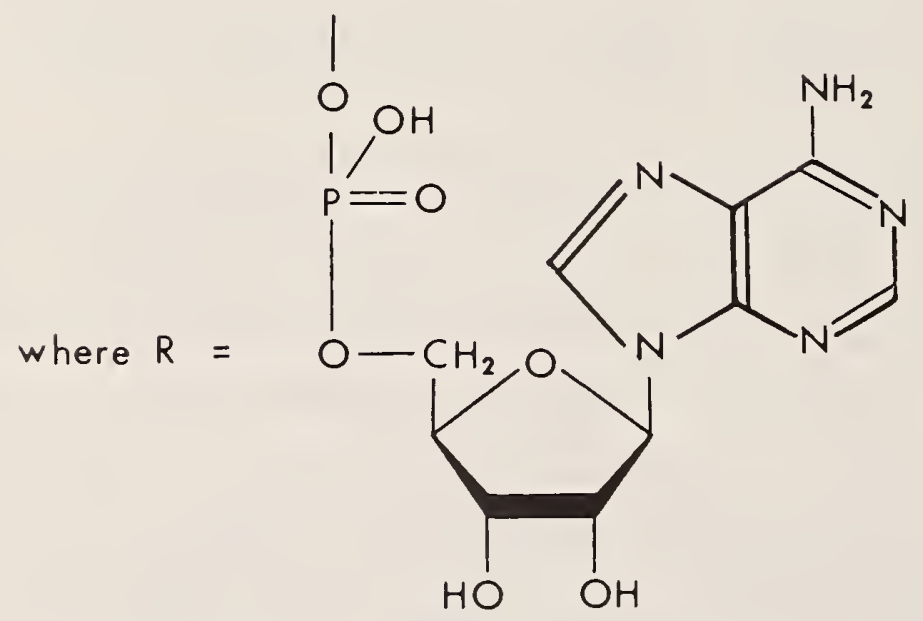

isolated and identified in 1936, it was not crystallized as the free acid until 1964. As far as we are aware, physical criteria for highly purified $\beta-N A D$ have not been reported in the literature. 
Similarly, although purification of $\beta-N A D H$ was vastly improved in 1961 by use of column chromatography on DEAFcellulose, physical criteria for the highly purified compound have apparently not been published. For example, as may be seen from table 17, the most recent value for $\varepsilon_{m M}$ at $340 \mathrm{~nm}$ is 6.25 (determined in 1957), but the value of 6.22 (determined in 1948) is usually cited.

Table 17. Ultraviolet absorption peaks of $\beta-N A D H$ at $p H 10$ $\varepsilon_{\mathrm{m} M}$ at $260 \mathrm{~nm} \varepsilon_{\mathrm{m} M}$ at $340 \mathrm{~nm} \quad A_{260} / A_{340} \quad$ References

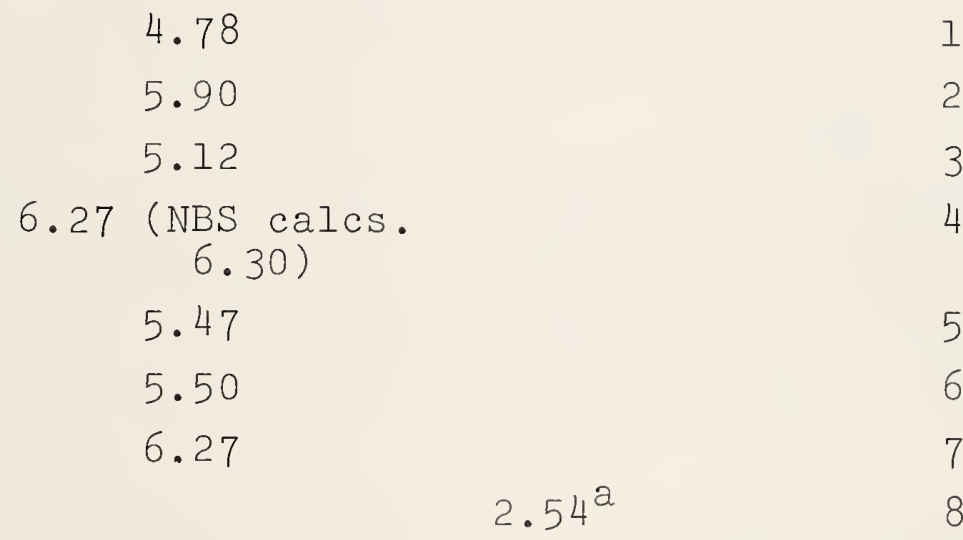

$5.93-6.31$ (av. 6.18 ) but they gave 6.22

6.3

10

16.9

$2.72-2.87^{b}$

11

6.25

12

16

$2.56^{c}$

13

14.4

$2 \cdot 32^{\mathrm{b}}$

14

$2 \cdot 4^{\mathrm{b}}$

15

14.0 (or

slightly lower)

2.24 or less ${ }^{\mathrm{b}} \quad 16$

2. 32

17

2. 32

18

14.4

6.2

2.32

19

$\overline{a_{\text {Ass umed }} \varepsilon_{340}}=6.27$. b Assumed $\varepsilon_{340}=6.22 . \quad c_{\text {Assumed }}$ $\varepsilon_{340}=6.25$ (average of values in Refs. 4, 9, and 12). 
1. Warburg, O., Christian, W., Biochem. Z. 287, 291 (1936).

2. Haas, E., Biochem. Z. 291, 79 (1937).

3. Negelein, E., Wulff, H. J., Biochem. Z. 293, 351 (1937).

4. Ohlmeyer, P., Biochem. Z. 297, 66 (1938).

5. Negelein, E., Brömel, H., Biochem. Z. 301, 135 (1939); 303, 132 (1939).

6. Schlenk, F., J. Biol. Chem. 146, 619 (1942).

7. Kubowitz, F., Ott, P., Biochem. Z. 314, 94 (1943).

8. Drabkin, D. L., J. Biol. Chem. 157, 563 (1945).

9. Horecker, B. L., Kornberg, A., J. Biol. Chem. 175, 385 (1948).

10. Colowick, S. P., footnote 4 on p. 389 of Ref. 9.

11. Lehninger, A. L., Biochem. Prepn. 2, 92 (1952).

12. Wallenfels, K., Christian, W., Methods Enzymol. 3, 882 ( 1957).

13. Rafter, G. W., Colowick, S. P., Methods Enzymol. 3, 887 (1957).

14. Siegel, J.M., Montgomery, G. A., Bock, R. M., Arch. Biochem. Biophys. 82, 288 (1959).

15. Dalziel, K., Biochem. J. 844, 240 (1962).

16. Silverstein, E., Anal. Biochem. 12, 199 (1965).

17. McComb, R. B., Gay, R. J., Clin. Chem. 14, 754 (1968).

18. Härtel, A., Helger, R., Lang, H., Z. Klin. Chem. Klin. Biochem. 6 , 259 (1968).

19. Specifications and Criteria for Biochemical Compounds, Tipson, R. S., (Ed.) Publication 1344, National Academy of Sciences-National Research Council, Washington, D. C., 1967, CoE-7, p. 207. 
Our initial studies have been made on the oxidized form $(\beta-N A D)$, because it has been reported to be more stable than the reduced form. Moreover, owing to its high hygroscopicity, studies were made with a single sample before the techniques developed were applied to other samples of $\beta-N A D$ and to samples of $\beta-N A D H$ (which is reported to be highly unstable to both moisture and light). The following preliminary results have been obtained with commercial samples of $\beta$-nicotinamide adenine dinucleotide.

1. Loss of Weight on Drying

(A. Cohen and A. J. Fatiadi)

On being dried at $25^{\circ} \mathrm{C} / 0.1$ torr, a sample (299.875 mg) of $\beta-N A D$ (lot no. 2331) lost the following decrements in weight (in $\mathrm{mg}$ ) after various total time-intervals (given, in hours, in parentheses): 1.7 (1), 0.4 (2), 1.1 (20), 0.3 (24), $0.15(26), 0.06$ (28), and 0.02 (30). Total loss in weight in $30 \mathrm{~h}, 3.73 \mathrm{mg}(1.24 \%)$. Further drying was not carried out.

After 2 days of drying under the same conditions, lot 2459 (2 samples of $\sim 350 \mathrm{mg}$, accurately weighed) lost 4.9 and $4.8 \%$; lot V3730 (3 samples of $\sim 100 \mathrm{mg}$ ) lost 2.3, 2.3, and $2.3 \%$.

The theoretical values for $\mathrm{C}_{21} \mathrm{H}_{2} \mathrm{~N}_{7} \mathrm{O}_{14} \mathrm{P}_{2}$ are as follows: anhydrous, $0.0 ; 1 \mathrm{H}_{2} \mathrm{O}, 2.6 ; 2 \mathrm{H}_{2} \mathrm{O}, 5.2 ; 2.5 \mathrm{H}_{2} \mathrm{O}, 6.4 ; 3 \mathrm{H}_{2} \mathrm{O}$, 7.5 ; and $4 \mathrm{H}_{2} \mathrm{O}, 9.8 \%$.

2. Elemental Analysis

The results of analysis of a commercial sample of $\beta-N A D$ are given in table 18 . 
Table 18. Analysis of a commercial sample (lot no. 2331) of $\beta-N A D$

Calculated for

$\mathrm{C}_{21} \mathrm{H}_{27} 7^{\mathrm{N}} 7^{\mathrm{O}} 14^{\mathrm{P}} 2$
C
H
$N$
Ash

\begin{tabular}{|c|c|c|c|c|c|c|}
\hline anhy & ydrous & 38.0 & 4.1 & 14.8 & 9.3 & 0.0 \\
\hline 1 & $\mathrm{H}_{2} \mathrm{O}$ & 37.0 & $4 \cdot 3$ & 14.4 & 9.1 & 0.0 \\
\hline 2 & $\mathrm{H}_{2} \mathrm{O}$ & 36.1 & 4.5 & 14.0 & 8.9 & 0.0 \\
\hline 2.5 & $\mathrm{H}_{2} \mathrm{O}$ & 35.6 & 4.6 & 13.8 & 8.7 & 0.0 \\
\hline 3 & $\mathrm{H}_{2} \mathrm{O}$ & 35.2 & 4.6 & 13.7 & 8.6 & 0.0 \\
\hline 4 & $\mathrm{H}_{2} \mathrm{O}$ & 34.3 & 4.8 & 13.3 & 8.4 & 0.0 \\
\hline
\end{tabular}

Found

not dried

36.3

4.7

13.6

8.1

2.5

By emission spectrographic analysis of a sample (lot no. 2331), the following semiquantitative estimates were obtained: Al, 50; $\mathrm{B}=5$; Ca, 100; Cr, 30; Cu, 40; Fe, 70; $\mathrm{Mg}, 20 ; \mathrm{Na}, 2000 ; \mathrm{Ni}, 25$; and $\mathrm{Si}, 50 \mathrm{ppm}$ of the sample.

\section{Paper Chromatography of $\beta-N A D$}

(A. Cohen)

Chromatograms of $\beta-N A D$ on whatman No. 1 chromatography paper were obtained by use of the following developers [16]: solvent $A, 66: 1: 33(\mathrm{v} / \mathrm{V})$ isobutyric acid-conc. ammonium hydroxide-water, $\mathrm{pH} 3.7 ; \mathrm{B}, 7: 3$ (v/v) 95\% ethanol-1.0 $M$ ammonium acetate, $\mathrm{pH} 7.5 ; \mathrm{C}, 2: 1$ ( $/ \mathrm{v}$ ) pyridine--water [17]; and D, 3:I (v/v) acetone-water [18]. $\beta-N A D$ and each of the reference compounds were dissolved in $1: 3(\mathrm{v} / \mathrm{v})$ ethanol-water, and the solutions were individually spotted in the following amounts: "adenosine diphosphoribose" sodium salt (ADPR), 2.4-4.8 $\mu g ; \alpha-N A D, 1.35-2.70 \mu g$; nicotinamide, $7.5 \mu g ; \beta-N A D H, 4-8 \mu g$; and $\beta-N A D, 27,135$, and $270 \mu \mathrm{g}$, by use of Drummond, disposable micro-p.pets. 
After development of the chromatograms, the compounds were detected by their fluorescence-quenching effect when irradiated with 254-nm light; 366-nm light failed to reveal any of these compounds, except $\beta-N A D H$. A spot corresponding to nicotinamide, used as reference compound, was evident with

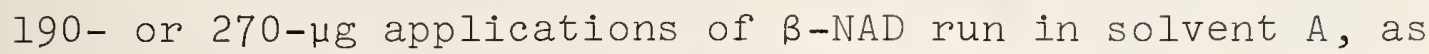
well as in solvent $B$. The $R_{F}$ values of the various compounds in each of these solvents are listed in table 19. Whether any of the reference components are impurities initially present in the $\beta-N A D$ or may be present as artifacts formed at the $\mathrm{pH}$ employed for the paper chromatography is still under study .

To maximize the possibility of finding impurities that might not be visibilized by quenching under 254-nm light, an alternative detection method was sought. A very useful procedure employed a spray consisting of methanolic potassium hydroxide (15 $\mathrm{g}$ in $100 \mathrm{ml}$ of methanol). Our exploration of this spray formulation was based on the observation of Kaplan et al. [20] that aqueous $5 \mathrm{M}$ sodium hydroxide induces in $\beta$-NAD a blue fluorescence that is forty times as intense as that caused by $M$ potassium cyanide. The alkali-induced fluorescence of $\beta$-NAD had been discovered earlier [21]. We tried potassium hydroxide $(5 M)$ as a chromatographic detection reagent but found that it causes some curling of the paper chromatograms. Our methanolic spray, when applied at $25{ }^{\circ} \mathrm{C}$, gives a highly fluorescent product with $\beta$-NAD under 366-nm irradiation. The $R_{F}$ values, and fluorescence with this spray (in parentheses), were as follows: Solvent A, nicotinamide, 0.87 (none); $\alpha$-NAD, 0.34 (blue); ADPR.Na salt, 0.20 (none); solvent $B$, nicotinamide, 0.74 (none); ADPR.Na salt, 0.17 (none); $\alpha-N A D, 0.10$ (blue); and $\beta-N A D H$ (which has an inherent blue fluorescence), 0.15 (blue). 
Table 19. Paper chromatography of samples ${ }^{a}$ of commercial B-NAD. Detection by fluorescence-quenching or $\mathrm{KOH}$-spraying, or both

\begin{tabular}{|c|c|c|c|c|c|}
\hline \multirow{2}{*}{$\begin{array}{l}\text { Sol- } \\
\text { vent }\end{array}$} & \multicolumn{2}{|c|}{ Manufacturer's } & \multirow{2}{*}{$\begin{array}{c}\text { lot no. } \\
2459\end{array}$} & \multirow{2}{*}{$\begin{array}{l}\text { Fluor- } \\
\text { escence } \\
\text { with } \mathrm{KOH}\end{array}$} & \multirow[t]{2}{*}{ Identification } \\
\hline & 2331 & V3730 & & & \\
\hline & 0.87 & 0.86 & 0.85 & none & $\begin{array}{c}\text { nicotinamide } \\
\text { (?) }\end{array}$ \\
\hline & $\begin{array}{l}0.45 \\
0.37 \\
0.21 \\
{[0.21]^{\mathrm{e}}} \\
0.13 \\
0.07 ?\end{array}$ & $\begin{array}{l}0.71 ? \\
0.44 \mathrm{c} \\
0.37 \\
0.21 \\
{[0.21]^{e}} \\
0.13 \\
0.07 ?\end{array}$ & $\begin{array}{l}0.71 ? \\
0.45 \\
0.38 \\
0.22 \\
{[0.22]^{e}} \\
0.14 \\
0.08 ?\end{array}$ & $\begin{array}{l}\text { none } \\
\text { none } \\
\text { blue } \\
\text { none } \\
\text { blue } \\
\text { blue } \\
\text { blue }\end{array}$ & $\begin{array}{l}\text { trace, unknown } \\
\text { unknown } \\
\text { B-NADd } \\
\text { ADPR } \\
\text { unknown } \\
\text { unknown } \\
\text { trace, unknown }\end{array}$ \\
\hline${ }^{f}$ & $\begin{array}{c}0.71 \mathrm{Vw}^{\mathrm{g}} \\
0.29 \mathrm{vw}^{\mathrm{g}} \\
0.18 \\
0.11 \\
\text { none } \\
0.02\end{array}$ & $\begin{array}{l}0.71 \mathrm{vw} g \\
0.28 \mathrm{vw} \\
0.17 \\
0.10 \\
{[0.10]^{\mathrm{h}}} \\
0.02\end{array}$ & $\begin{array}{l}0.71 \mathrm{vw}^{\mathrm{g}} \\
0.28 ? \mathrm{vw} \\
0.18 \\
0.12 \\
\text { none } \\
0.10\end{array}$ & $\begin{array}{l}\text { none } \\
\text { none } \\
\text { none } \\
\text { blue } \\
\text { blue }\end{array}$ & $\begin{array}{l}\text { nicotinamide } \\
\text { unknown } \\
\text { ADPR } \\
\text { B-NAD } \\
\text { unknown } \\
\text { unknown }\end{array}$ \\
\hline
\end{tabular}
application of $240 \mathrm{\mu g}$.

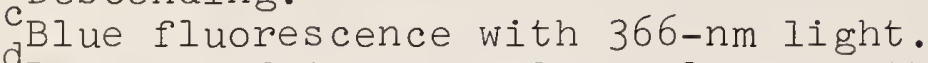

Because of its $R_{F}$ value, close to that of $\beta-N A D$, the possibility of presence of $\alpha$-NAD cannot be excluded.

Visibilized only by spraying with $\mathrm{KOH}$; blue fluorescence in interior portions of ADPR spot; tailing (blue fluorescence) fo slower spot.

As cending.

Every weak (by quenching).

${ }^{\mathrm{h}}$ A fluorescent impurity, having the same $R_{F}$ value as $B-N A D$, seen (only in this sample) under 366-nm lîght; impure $\beta-N A D$ can contain [19] a fluorescent impurity that is not $\beta-N A D H$. 
We have found our detection spray to be very sensitive and helpful in differentiating impurities associated with $\beta-N A D$ and $\beta-N A D H$. The blue fluorescence given on spraying does not appear immediately on the chromatogram, but only begins to be visible after $\sim 2 \mathrm{~min}$ and has increased substantially after $210-15 \mathrm{~min}$. The limit of sensitivity of the spray has not yet been determined; however, in one test, $4 \mu g$ of a commercial sample of $\beta$-NADH, developed with solvent $C$ and apparently pure by inspection under 366-nm light, was found with the spray reagent to exhibit another fluorescent spot, possibly $\beta-N A D$.

In solvent $B, A D P R$ is not well separated from $B-N A D$, but may overlap it partially; however, their discrimination presents no difficulty when the detection spray is used.

In all four solvent systems, $\alpha$-NAD cannot be resolved from $\beta-N A D$ if the proportion of the latter far exceeds that of the former, and so, if the $\alpha$-were present as an impurity in $\beta-N A D$, the $\alpha-N A D$ would not be detected.

With solvent $A$, lot V3730 of $\beta-N A D$ under 366-nm light showed a fluorescent impurity that was not found in other commercial samples examined. Lots 2331 and 2459, on the other hand, contained an impurity with this same $R_{F}$ value, but it was nonfluorescent. With solvent $B$, this fluorescent impurity in lot V3730 was not resolved from the B-NAD.

Descending paper chromatography [17] of the reference compounds for $24 \mathrm{~h}$ with solvent $C$ showed $R$ values (relative to $R=1$ for $\beta-\mathrm{NADH}$ ) and fluorescence with the spray (in parentheses) as follows: ADPR.Na salt, 0.93 (none); $\alpha-N A D$, 0.74 (blue); $\beta-N A D H$ has an inherent blue fluorescence. Nicotinamide was also used as a reference compound, but was not detected because, under these conditions, it was eluted from the chromatogram. The results of descending paper chromatography on commercial $\beta-N A D$ (lot 2331) in solvent $C$ are given in table 20. Results with solvent $D$ were similar to those in $C$. 
Table 20. Paper chromatography ${ }^{a}$ of $\beta-N A D$ ( lot no. 2331) Solvent $\quad R^{\mathrm{b}}$

Fluorescence with $\mathrm{KOH}$ none

none

blue

blue

none

none

blue

blue

Identification

unknown

ADPR

$\beta-N A D^{d}$

unknown

unknown

unknown

$\beta-N A D d$

unknown

\footnotetext{
application of $268 \mu \mathrm{g}$.

Relative to $R=1$ for $\beta-\mathrm{NADH}$.

Descending $(24 \mathrm{~h})$.

dor $\alpha$-NAD, or both.
}

For descending paper-chromatography in solvent $D$, the $R$ values (relative to $R=1$ for $\beta-\mathrm{NADH}$ ), and fluorescence with the spray (in parentheses), were: ADPR.Na salt, 1.02 (none); $\alpha-\mathrm{NAD}, 0.75$ (blue); $\beta-\mathrm{NADH}$ has an inherent blue fluorescence. A commercial sample (lot 2331) of B-NAD showed three impurities in solvent $D$ (see table 20). Here $A D P R \cdot N a$ was not evident, but a very fast-moving impurity $(R=1.14)$ was evident by its fluorescence-quenching. Surprisingly, the $\beta-N A D$ spot showed an unexpected blue fluorescence under 366-nm light. In some way, this may be related to the complex with acetone that $\beta-N A D$ has been reported [21] to form in alkaline medium.

Attempts to resolve $\alpha-N A D$ from $\beta-N A D H$ by t.l.c. on PEIcellulose with solvent $B$ or with $0.2 M$ Tris buffer ( $\mathrm{pH}$ 9.0) were unsuccessful. 
B-NADH examined by descending chromatography on paper with solvent $C$ appeared to be pure under 254- and 366-nm irradiation, but exhibited a fluorescent impurity at $\kappa_{F} 0.50$ when sprayed with the methanolic KOH. B-NAD run as a reference standard at a nearby position showed $R_{F} 0.51$.

\section{Gas-liguid Chromatography of Commercial B-NAD after Per(trimethylsilyl)ation}

(A. J. Fatiadi)

A sample ( $5 \mathrm{mg}$ ) of commercial $\beta$-NAD (lot no. 233l) was per(trimethylsilyl)ated by use of $0.5 \mathrm{ml}$ of Tri Sil Z at $25{ }^{\circ} \mathrm{C}$ for $60 \mathrm{~min}$ and $24 \mathrm{~h}$. The products were injected into a glass column ( $6 \mathrm{ft} . \mathrm{x} 2 \mathrm{~mm}$ I.D.) having Chromosorb $\mathrm{Q}$ (60-80 mesh) as the solid support, with a liquid phase of $3 \%$ of OV-17. The column was operated isothermally at $150{ }^{\circ} \mathrm{C}$, and the components were detected with a flame-ionization detector operated at $200{ }^{\circ} \mathrm{C}$. The sample size was $10 \mu \mathrm{g}$. The retention times observed for the components detected are given in table 2l. Work is still in progress on the identification of the components.

Table 21. Retention times of products obtained by per(trimethylsilyl)ation of commercial $B-N A D$ ( lot no. 2331)

Approximate retention time (minutes) of components observed after ${ }^{a}$

1 hour

$$
\begin{array}{r}
3.6 \\
4.4 \\
7.2 \\
9.6 \\
15.2 \\
24.0
\end{array}
$$

24 hours

3.6

7.0

9.4

14.8

23.8

38.4

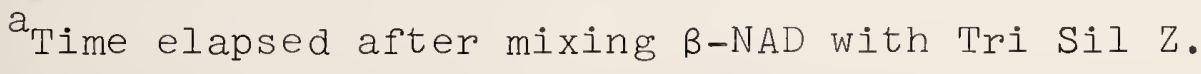


Approximately 3---4 mg (carefully weighed) of commercial $\beta$-NAD was rapidly dissolved in water or in tris(hydroxymethyl)aminomethane (Tris) buffer, adjusted to pH 8 with glacial acetic acid; total volume $100 \mathrm{ml}$. A sample, dried under high vacuum without heat, was similarly aissolved. Ultraviolet spectra of the solutions were recorded from 600 to $220 \mathrm{~nm}$ by use of a Cary Model $14 \mathrm{u} \cdot \mathrm{v}$. spectrophotometer. (The spectra showed no absorption from 600 to $350 \mathrm{~nm}$.$) The solutions were then stored in the dark at$ 20--25 ${ }^{\circ} \mathrm{C}$, and re-examined periodically. No change was apparent in 3 days. The data are given in table 22.

Table 22. Ultraviolet spectral data for B-NAD (lot no. 2331)

\begin{tabular}{|c|c|c|c|c|c|}
\hline \multirow[t]{2}{*}{ Solvent } & \multirow{2}{*}{$\begin{array}{l}\text { Time at } \\
25{ }^{\circ} \mathrm{C} \text { (days) }\end{array}$} & \multicolumn{2}{|c|}{$\varepsilon_{m M}$} & \multirow{2}{*}{$A_{250} / A_{260}$} & \multirow{2}{*}{$A_{280^{\prime}} A_{260}$} \\
\hline & & $\begin{array}{l}\text { At } \\
231.5 \\
\mathrm{~nm}\end{array}$ & $\begin{array}{r}\text { At } \\
260 \\
\mathrm{~nm}\end{array}$ & & \\
\hline \multirow[t]{3}{*}{ Water } & Not dried & 8.01 & 17.5 & 0.83 & 0.24 \\
\hline & 3 & 8.15 & 17.5 & 0.82 & 0.23 \\
\hline & 51 & decon & osed & & \\
\hline \multirow{5}{*}{$\begin{array}{l}\text { Tris buffer } \\
(\mathrm{pH} 8)\end{array}$} & 0 & 7.86 & 17.4 & 0.82 & 0.21 \\
\hline & 3 & 7.89 & 17.4 & 0.83 & 0.21 \\
\hline & $\begin{array}{c}\text { Dried } \\
0\end{array}$ & 7.85 & 17.5 & 0.82 & 0.21 \\
\hline & 18 & 7.67 & 17.6 & 0.80 & 0.20 \\
\hline & 41 & 7.29 & 17.35 & 0.79 & 0.20 \\
\hline & Manuf. data & -- & 17.6 & 0.82 & 0.21 \\
\hline oH 7 & NRC [22] & -- & 18.0 & 0.83 & 0.22 \\
\hline
\end{tabular}


6. Enzymic Assay of $\beta-N A D$

(A. Cohen)

When $\beta-N A D$ is reduced, a peak characteristic of $\beta-N A D H$ appears at $340 \mathrm{~nm}$, and the initial absorbance at $260 \mathrm{~nm}$ is diminished in intensity. The NRC values of $\varepsilon_{m M}$ for the reduced compound are 14.4 at $260 \mathrm{~nm}$ and 6.2 at $340 \mathrm{~nm}$ [22] (see table 17). It is commonly accepted that lower values of $A_{260} / A_{340}$ are indicative of purer $\beta-N A D H$; consequently, it would also be indicative of a purer $\beta-N A D$ as the reactant.

Horse-liver alcohol dehydrogenase (HLAD), $2.3 \mathrm{U} / \mathrm{mg}$ [23], was used. Commercial B-NAD (lot no. $2331 ; 4.811 \mathrm{mg}$ ), $40 \mathrm{ml}$ of $0.5 M$ ethanol in Tris buffer ( $0.1 M$ unadjusted; $\mathrm{pH} \sim 9.8$ ), and $2 \mathrm{mg}$ of HLAD in $2 \mathrm{ml}$ of Tris buffer were mixed, and diluted to $100 \mathrm{ml}$ with Tris buffer. The blank was a comparable solution without $\beta-N A D$. The reaction reached completion in 38--50 min, and was read at $75 \mathrm{~min}$. Absorbances were measured at $340 \mathrm{~nm}$, at $260 \mathrm{~nm}$, and at two minima. The solution was then left exposed to laboratory light at room temperature, and was re-examined after 4, 8, and 18 days. The results are summarized in table 23. 
Table 23. Enzymic assay of B-NAD (lot no. 2331) by reduction with ethanol in the presence of horse-liver alcohol dehydrogenase

$\begin{array}{cl}\varepsilon_{m M} a t^{a} & \text { NRC } \\ (n m) & \text { constants } \\ & {[22] \text { for }} \\ & \text { B-NADH }\end{array}$

Time kept at $25^{\circ} \mathrm{C}$ (days)

$\sim 235$

14.4

6.74

6.82

6.80

6.76

260

290

340

$6 \cdot 2^{\mathrm{b}}$

14.6

14.7

14.7

15.0

1.39

1.51

1.49

1.92

Absorb-

ance

ratio

$A_{250} / A_{260}$

0.82

5.85

5.88

5.71

5.61

$A_{280} / A_{260}$

0.23

0.80

0.80

0.79

0.79

$A_{260} / A_{340}$

2.32

0.25

0.25

0.26

0.27

2.49

$2.50 \quad 2.58$

2.68

a For resulting $\beta$-NADH.

Manufacturer's data for this lot, $5.9 \pm 0.2$. 
2. CARBOHYDRATE SYNTHESIS, STRUCTURE, AND CHARACTERIZATION: NOVEL RESEARCH MATERIALS AND MODEL COMPOUNDS

The Section's area of research specialization in organic chemistry continues to be the carbohydrates. Two ketoses were examined: D-fructose and D-psicose, the former because its 1,2:4,5-di-0-isopropylidene derivative lacked sufficient purity to be used unequivically in subsequent, synthetic experimentation. The nature of the problem with its purity was resolved and, as an outgrowth, a higheryield process for making the pure derivative was developed. Work with D-psicose was related to this. Only by use of that pure D-fructose derivative (i.e., free of the contaminating 2,3:4,5-di-0-isopropylidene isomer) was a pure D-psicose obtainable; by utilizing the pure "diacetonefructose" as the starting material, confounding aspects in publications from several laboratories on the synthesis of D-psicose were clarified.

Proton magnetic resonance studies of interesting carbohydrates were actively pursued during the year. Although there is considerable interest in understanding the relative importance of chair and nonchair conformations of molecules that contain six-membered rings, there is a paucity of proton resonance data for unequivocal examples of nonchair conformations that can serve as a reference for conformational equilibria. Certain rigid carbohydrate molecules can be most useful in this regard. Our p.m.r. study of 3-O-benzoyl-1,2,4-0-benzylidyne- $\alpha$-D-ribopyranose has now defined the degree of applicability of previously developed relationships between coupling constants and dihedral angles with the locked, skew conformation of this compound. 
A p.m.r. study was also conducted on 1,2-0-alkylideneD-glucopyranoses to settle any controversy concerning their adopting a possible skew conformation. The resonance parameters found by our study showed clearly that the compounds possess chair conformations.

Computer programs have been of considerable importance for the analysis of complex p.m.r. spectra. Now, too, the Section possesses a high-resolution spectrometer having heteronuclear resonance capability, and its use is extending the range of our work.

Inosose oxidation by the Somogyi reagent was examined, and led to the examination of the reactant in this and other copper-complex reagents by electron-spin resonance. The reaction with the Somogyi reagent appears to involve radical or one-electron mechanisms, depending on the reaction temperature. A facile synthesis of inositol hexasulfate has been developed. This compound is of interest, since it is the sulfuric acid analog of phytic acid, a naturally occurring myo-inositol derivative.

\section{A Synthesis of the Isomeric Di-O-isopropylidene- - -D-} fructopyranoses

$$
\text { (R. F. Brady, Jr.) }
$$

1. Introduction

Large amounts of $1,2: 4,5$-di-O-isopropylidene- $\beta$-Dfructopyranose (2) were required as the starting material for a synthetic program leading to crystalline derivatives of rare sugars. Samples of this diacetal were therefore purchased from commercial sources, and, for comparison, samples were synthesized by published procedures. By per(trimethylsilyl)ation followed by g.l.c. analysis, it was found that most of these samples were impure, the contaminant of 2 being its isomer, namely, 2, 3:4,5-di-0isopropylidene- $\beta-\underline{\underline{D}}$-fructopyranose $(\underset{\sim}{3})$. A procedure was 
therefore sought for freeing the 2 from this impurity, with the highest possible recovery of pure $\underset{\sim}{2}$.

In addition, as the literature on the synthesis of 2 records yields lying only in the range of 50 [24] to 66\% [25], it was desired to attempt to devise a method that would give pure 2 in higher yield.

\section{Results and Discussion}

A suspension of crystalline $\underset{\equiv}{D}$-fructose $(\underset{\sim}{(})$ in acetone containing sulfuric acid (as catalyst) was stirred, and the reaction was monitored after various time-intervals by g.l.c. analysis of per(trimethylsilyl)ated, neutralized aliquots; the diacetal observed first was compound $\underset{\sim}{2}$.

It was found that, at high concentrations of catalyst $(\geq 5 \%), \underset{\sim}{2}$ isomerizes rapidly to $\underset{\sim}{3}$. The concentration of $\underset{\sim}{3}$ is maximal at equilibrium; indeed, $\underset{\sim}{3}$ is isolable in satisfactory yield if the reaction is allowed to reach this stage.

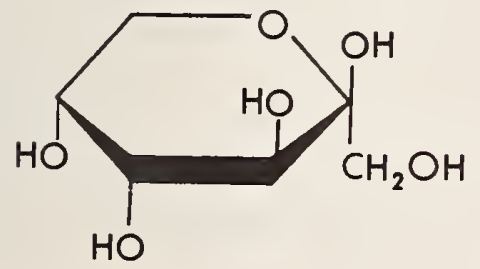

1

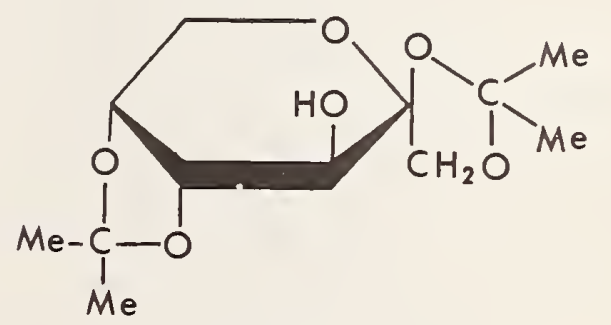

2

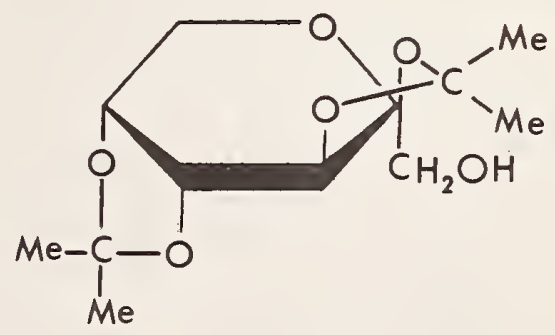

3 
In contrast, at low concentrations of catalyst $(\leqslant 0.5 \%)$, compound $\underset{\sim}{2}$ slowly isomerizes to $\underset{\sim}{3}$. By arresting the reaction at the point at which the concentration of 2 is maximal, compound 2 was isolated in $80 \%$ yield.

Use of intermediate concentrations of catalyst, or premature processing of the reaction mixture, affords mixtures of diacetals 2 and 3 , such as those reported by ohle and Koller [26] and $\tilde{B} e l l$ [25]. Such mixtures are more difficult to separate, and isolation of either isomer in pure form is attended by great diminution in yield.

Apparently, $\underset{\sim}{2}$ is the product of the kinetically controlled condensation of D-fructose with acetone; that it is observed first is, presumably, a consequence of the higher reactivity of the primary hydroxyl group on $\mathrm{C}-\mathrm{I}$, as compared with that of the secondary hydroxyl group on $\mathrm{C}-3$. However, in 2 , the spiro-fusion of the 1,3dioxolane ring to the pyranoid ring creates strain [27] at $\mathrm{C}-2$. This strain is relieved when 2 rearranges to 3, because the 1,3-dioxolane ring becomes cis-fused to the pyranoid ring, and diacetal $\underset{\sim}{3}$ greatly preponderates in the mixture at equilibrium. Thus, in acetone containing 5\% of sulfuric acid, each pure isomer is converted, within 5 min at room temperature, into an equilibrium mixture consisting of $94 \%$ of $\underset{\sim}{3}$ and $6 \%$ of $\underset{\sim}{2}$; hence, $\underset{\sim}{3}$ j.s thermodynamically more stable than 2 .

These results have been incorporated in an article which has been accepted for publication [28].

B. Synthesis of $\underline{\underline{D}}$-Psicose

(R.S. Tipson, R. F. Brady, Jr., and B. F. West)

1. Introduction

In Technical Note 457, we described preliminary studies

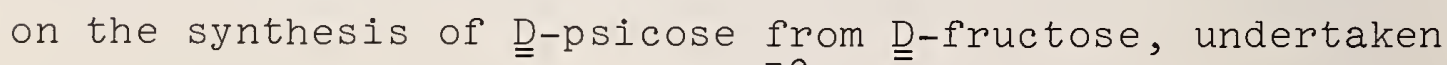
58 
because a crystalline derivative of D-psicose was desired for issuance as a Standard Reference Material. We have, in particular, re-investigated the preparation of its 1,2:4,5-di-0-isopropylidene derivative, which is crystalline.

D-Psicose (D-ribo-hexulose, $\underset{\sim}{5}$ a) is a rare sugar that occurs in the antibiotic psicofuranine [29] ( $9-\beta-D-$ psicofuranosyladenine) and in Itea plants [30]; it has also been found in the nonfermentable fraction of cane molasses [31], where it may have been formed by the action of the lime used in sugar manufacture. D-Psicose was first synthesized [32] in 1936, by the pyridine-catalyzed isomerization of D-allose; it has also been prepared by a 6-step synthesis [33] from D-ribose, by a 4-step synthesis [34] from D-fructose, by mild isomerization of D-glucose with alkali [35], and by isomerization of D-fructose by $N, N^{\prime}$-dicyclohexylcarbodiimide in hot methanol [36] to D-psicose, D-glucose, and $\underline{\underline{D}}$-mannose, followed by fermentation with bakers' yeast. However, some of these methods require expensive starting-materials, and all of them are tedious and give low overall yields.

In 1967, McDonald [37] described a new synthesis of the sugar, starting from $\underline{\underline{D}}$-fructose ( $\underset{\sim}{\mathrm{l} a}$ ), that involved the following steps. Compound $\underset{\sim}{\mathrm{la}}$ was converted into $1,2: 4,5$-di-

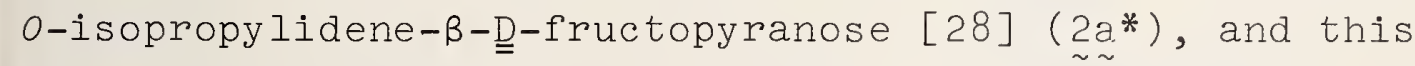
was oxidized with methyl sulfoxide-acetic anhydride (oxidant I) to $1,2: 4,5$-di-0-isopropylidene- $\beta$-D-erythro-2,3hexodiulo-2,6-pyranose ( $\underset{\sim}{3}$ ); it was stated that the 3 -acetate $(\underset{\sim}{2 c})$ of compound $\underset{\sim}{2 a}$ was simultaneously formed, but that recrystallization of the crude product afforded pure $\underset{\sim}{3}$. Reduction of 3 with sodium borohydride in aqueous methanol then gave $1,2: 4,5$-di-O-isopropylidene- $\beta-D-r i b o-h e x u l o p y r a n o s e$

*Takagi and Rosenstein [38] have found that, in crystalline $\underset{\sim}{2 a}$, the conformation of the molecules is intermediate between $1 C(\underline{\underline{D}})$ and $H_{O}^{2}(\underline{\underline{D}})$. 


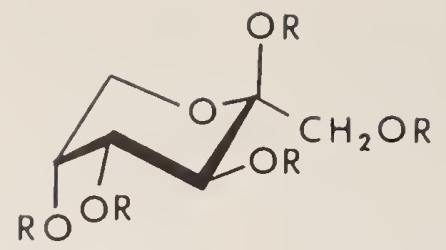

1

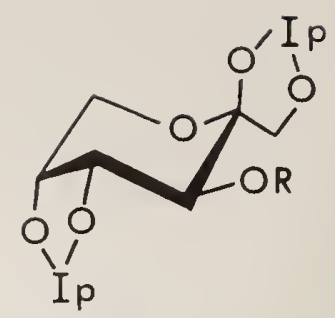

2 $+\quad(2 c, R=A c)$

$2 \mathrm{~d}, \mathrm{R}=\mathrm{CH}_{2} \mathrm{SMe}$

3

$\mathrm{NaBH}_{4}$

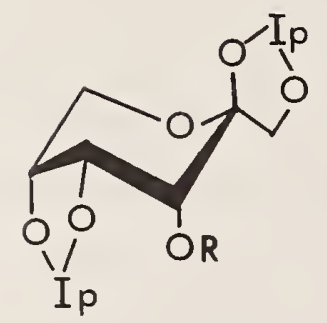

4
$\stackrel{\mathrm{H}^{+}}{\longrightarrow}$

5
a, $R=H$
b, $\mathrm{R}=\mathrm{SiMe}_{3}$ 
( $\sim \sim \sim \sim \sim$ ) contaminated with "only a trace" of the corresponding D-fructose diacetal ( $\underset{\sim}{2 a})$, presumably removed on recrystallization of crude $\underset{\sim}{4} \underset{\sim}{\mathrm{a}}$; and acid hydrolysis of compound $\underset{\sim}{4}$ a afforded D-psicose $(\underset{\sim}{5} \underset{\sim}{a})$.

Because this synthesis provides a direct means for preparing D-psicose, it was decided to repeat this procedure to produce a supply of the crystalline $1,2: 4,5$-di-Oisopropylidene- $\beta$-D-psicopyranose ( $\sim_{\sim}$ ) for use as a Standard Reference Material. Three months after publication of McDonald's Note [37], a more detailed article describing similar work was published independently [39]; certain of the conclusions described in the latter article had already been arrived at by us [40]. Finally, in 1968, another article was published on the same subject [41]. The present work was done in order to explain certain discrepancies between the results of the three sets of workers [37,39,4I].

\section{Results and Discussion}

In our study, McDonald's procedure [37] for preparing 3 was first repeated in accordance with her instructions. Compound $\underset{\sim}{2 a}$ was oxidized during $24 \mathrm{~h}$ at $25{ }^{\circ} \mathrm{C}$ with a solution of acetic anhydride ( 1 molar proportion) in methyl sulfoxide ( 70 molar proportions), and crude compound 3 was isolated in a yield of $250 \%$ of the theoretical, as compared with the $70 \%$ yield of crude product reported by McDonald [37]. Because of this result, a series of experiments was performed in which the molar proportions of $\underset{\sim}{2 a}$ to acetic anhydride (A) to methyl sulfoxide (B) were $1: 2: 42$, $1: 16: 42$, and $1: 21: 42$, but the yield of 3 (recrystallized) was only raised to $52--54 \%$ of the theoretical. As the oxidation of 1 mole of $\underset{\sim \sim}{2 a}$ with McDonald's proportions would require the use of 5 liters of methyl sulfoxide, the molar proportions of $2 \mathrm{\sim} a \mathrm{~A}: \mathrm{B}$ were changed to $1: 10: 10$, in contrast to the ratios of $1: 1: 70$ [37], $1: 23: 46$ 
[39], and 1:43:85 [41] reported in the literature. The reaction was monitored by t.l.c. on Silica Gel G, and we found that (a) the time necessary for completion of the reaction with the 1:10:10 ratios was 4 days, as compared with 2 days [37] and 1 day [39,41]; and (b) only two products are formed. James and co-workers [39] reported that three products are formed, whereas the other workers [37,41] found only two.

In all of these experiments, we found that, accompanying compound $\underset{\sim}{3}$, there was a large proportion of a different crystalline compound (X) that, solely on the basis of $R_{F}$ values in t.l.c., had been regarded by NicDonald [37] as being the 3 -acetate $\underset{\sim}{2 c}$. (The rather close $R_{F}$ values of $X$ and $\underset{\sim}{2 c}$ in her solvent system explain the misidentification.) However, we found that the second product (X) shows no carbonyl absorption in its i.r. spectrum, and therefore could not be the acetate $\underset{\sim}{2 c}$. To verify this conclusion, we prepared an authentic specimen of acetate $\underset{\sim}{2 c}$ by the method of Fischer and Noth [42], and found that the i.r. spectrum of $\underset{\sim}{2 c}$, which shows bands at $1735(\mathrm{C}=0)$ and $1240 \mathrm{~cm}^{-1}(\mathrm{C}-0)$, is entirely different from that of compound $X$. A mixture of compound $X\left(\mathrm{~m} \mathrm{p} 82.5-83{ }^{\circ} \mathrm{C}\right)$ with compound $\underset{\sim}{2 \mathrm{c}}\left(\mathrm{m} p 76-77{ }^{\circ} \mathrm{C}\right)$ melted at $59^{\circ} \mathrm{C}$. Compound $X$ was identified [39] as $\underset{\sim}{2 d}$, the 3-(methylthio)methyl ether of compound $\underset{\sim}{2 \mathrm{a}}$.

To effect separation of the diulose acetal $\underset{\sim}{3}$ from $X(\underset{\sim}{2} \underset{\sim}{2})$, McDonald [37] recrystallized the crude product from methanol, to afford $3 \underset{\sim}{3}$; compound $\underset{\sim}{2 \mathrm{~d}}$ was not recovered. James and co-workers [39] isolated $\underset{\sim}{3}$ by recrystallization of the crude product from petroleum ether; the mother liquor was evaporated to dryness and the resulting syrup was crystallized from petroleum ether to give a mixture of crystalline ${ }_{\sim}$ (as needles) and $\underset{\sim}{2 d}$ (as rectangular plates). These were separated "mechanically," and recrystallization of the platelets from petroleum ether gave $\underset{\sim}{2 d}$. We have found that 
the two compounds are readily separated by dissolution of the mixture in absolute methanol ( $5 \mathrm{ml} / \mathrm{g}$ ) and addition of water $(2.5 \mathrm{ml} / \mathrm{g})$; the ether $\underset{\sim}{2 \mathrm{~d}}$ crystallizes out almost quantitatively and is filtered off, the mother liquor is evaporated to dryness, and this residue, crystallized from hexane, gives $\underset{\sim}{3}$.

Whereas the diulose acetal $\underset{\sim}{3}$ has been reported to have $[\alpha]_{D}-100.4^{\circ}$ (in acetone)[37], $-126.4^{\circ}$ (in chloroform)[39], and $-106^{\circ}$ (in ethanol) $[41]$, we found $[\alpha]_{D}^{25}-104.7^{\circ}$ (in acetone), $-119.2^{\circ}$ (in chloroform), and $-113.5^{\circ}$ (in ethanol). Because of these discrepancies, we decided to prepare compound $\underset{\sim}{3}$ by oxidation of $\underset{\sim}{2 a}$ with ruthenium tetraoxide [4I] (oxidant II) by an improved procedure [43]. Compound $\underset{\sim}{3}$, obtained in this way, was homogeneous by t.l.c. and g.l.c. analysis; it had the same specific rotations as those we had found for 3 prepared by use of oxidant $I$.

The reduction of pure compound $\underset{\sim}{3}$ was effected with sodium borohydride by a modification of the procedure of James and co-workers [39]. G.I.c. analysis of the 3-(trimethylsilyl) ether of the "crude" crystalline product, under conditions that would have detected the presence of $0.1 \%$ of compound $\underset{\sim}{2 b}$, revealed that the reduction product consisted solely of $\underset{\sim}{4 a}$. Hence, the reduction is completely stereospecific.

This result is not in agreement with that of the earlier workers. By t.l.c., McDonald [37] found "a trace" of $2 \underset{\sim}{2}$ in the product obtained on sodium borohydride reduction of 3 (prepared with oxidant I). Cree and Perlin [4I] reported that, on lithium aluminum hydride reduction of $\underset{\sim}{3}$ (prepared with oxidant II), "only a trace of the D-fructose derivative was formed during the reduction, and sodium borohydride in aqueous ethanol was almost as exclusively selective in favoring the production" of $\underset{\sim}{4 a}$. In contrast, on reduction of 3 prepared with oxidant I, James et al. [39] found, by g.l.c. analysis, $10 \%$ of $\underset{\sim}{2 a}$ in the crude $\underset{\sim}{4 a}$ obtained by 
reduction with lithium aluminum hydride, and $1.8 \%$ of $\underset{\sim \sim}{2 a}$ when 3 was reduced with sodium borohydride.

We have sought an explanation for these discrepancies. The possibility that $\underset{\sim \sim}{2 a}$, as a contaminant of $\underset{\sim \sim}{4 a}$, arose from $\underset{\sim}{2 \mathrm{~d}}$ as a contaminant of $\underset{\sim}{3}$ was excluded, as we found that $\underset{\sim}{2 \mathrm{~d}}$ is unaffected by sodium borohydride under the conditions used for reducing $\underset{\sim}{3}$ to $\underset{\sim}{4}$. Hence, the only explanation possible is that a small proportion of $\underset{\sim}{2 a}$ remained unchanged on oxidation of $\underset{\sim}{2 a}$, and that it was carried as a contaminant of $\underset{\sim}{3}$ (and, thence, of $\underset{\sim}{4 a}$ ); the presence of a small proportion of $\underset{\sim}{2 a}$ in $\underset{\sim}{3}$ would not be readily revealed by the t.I.c. procedures that had been used $[37,39,41]$.

In addition to $\underset{\sim}{3}$ and $\underset{\sim}{2}$, James et ar. [39] found, in the mixture obtained by treating $\underset{\sim}{2 a}$ with oxidant $I$ for 1 day, a third component that was not unoxidized $\underset{\sim}{2 a}$. It has been estabiished [28] that many commercial and laboratory samples of $\underset{\sim}{2 a}$ are contaminated with 2,3:4,5-di-0isopropylidene- $\beta-D-f r u c t o p y r a n o s e ~(\underset{\sim}{\sigma a})$. * We have now found that compound $\underset{\sim}{\sigma a}$ is converted into a mixture of $2,3: 4,5$-di-o-isopropylidene-aldehydo-B-D-arabino-hexosulo-
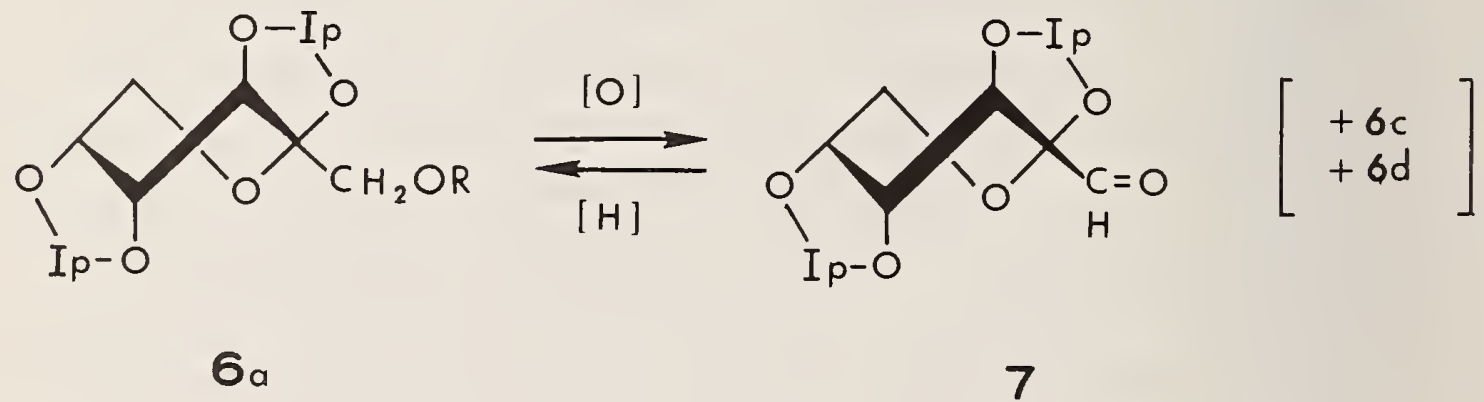

$$
7
$$
a, $R=H$
b, $\mathrm{R}=\mathrm{SiMe}_{3}$
c, $R=A C$
d, $\mathrm{R}=\mathrm{CH}_{2} \mathrm{SMe}$

*The $S_{4}^{6}$ conformation depicted was found by Maeda and co-workers [44]. 
2,6-pyranose (7) and the l-acetate $(\underset{\sim}{6 c})$ and

l-(methylthio)methyl ether (6d) of 2,3:4,5-di-o-

isopropylidene- $\beta-\underline{\underline{D}-f r u c t o p y r a n o s e}$ on treatment with oxidant I under the conditions that we had used for oxidation of compound $\underset{\sim}{2 a}$. In the t.l.c. developer used by James et al. [39], compound 7 has $R_{F} 0.39$, and compounds $\underset{\sim \sim}{6 c}$ and $\underset{\sim}{6 d}$ have $R_{F}$ 0.72; the latter is the $R_{F}$ value reported [39] for the "third product" detected in the oxidation of $\underset{\sim}{2 a}$ by oxidant I, showing that their $\underset{\sim}{2 a}$ might, indeed, have been contaminated with $\underset{\sim \sim}{6 a}$. The presence of a small proportion of $\underset{\sim \sim}{6 a}$ in $\underset{\sim}{2 a}$ would not be readily revealed by the t.l.c. procedures that had been used $[37,39,41]$.

Reduction of compound $\underset{\sim}{7}$, under the conditions that we had used for the reduction of compound $\underset{\sim}{3}$, afforded compound $\underset{\sim}{6 a}$. Thus, if $\underset{\sim}{6} \underset{\sim}{a}$ is a contaminant of $\underset{\sim}{2 a}$, it will give its oxidation product (7) as a contaminant of $\underset{\sim}{3}$; on reduction. of this mixture, $\underset{\sim}{6 a}$ will be a contaminant of $\underset{\sim}{4} \underset{\sim}{\mathrm{a}}$, and thence, D-fructose $(\underset{\sim}{(\mathrm{l}})$ will be a contaminant of the desired $\underline{\underline{\sim}}$-psicose $(\underset{\sim}{5} \mathrm{a})$. We therefore emphasize that, for preparation of pure $\underset{\sim}{4 a}$, the diacetal $\underset{\sim}{2 a}$ used as the starting material must be free from diacetal $\underset{\sim}{6} \underset{\sim}{a}$, and, before reduction, the diulose acetal $\underset{\sim}{3}$ must be free from $\underset{\sim}{2 a}$.

Such contamination of $\underset{\sim}{4 a}$ by $\underset{\sim}{2 a}$ or $\underset{\sim}{6 a}$ is most readily detected by hydrolysis of $\underset{\sim \sim}{4 a}$ to $\underset{\sim}{5 a}$, because $\underset{\sim}{5 a}$ has $[\alpha]_{D}+4.7^{\circ}$ (in water) [33], whereas $\underset{\sim}{\operatorname{la}}$ (from any $\underset{\sim}{2 a}$ or $\underset{\sim}{6 a}$ present as a contaminant) has $[\alpha]_{D}-92^{\circ}$ (in water) [45]. On hydrolysis of our samples of $4 a$ with 0.1 oxalic acid at $65^{\circ} \mathrm{C}$, the resulting solution of $\underset{\sim}{5 a}$ had $[\alpha]_{D}+4.7^{\circ}$ (in water) and the sugar was homogeneous by t.l.c. [27] and by g.l.c. of its per(trimethylsilyl) derivative $(\underset{\sim}{5} \underset{\sim}{b})$. The previous workers $[37,39,41]$ did not report having applied these tests to their samples of $\underset{\sim}{4 a}$. 
Freshly recrystallized $\underset{\sim}{4 a}$ had $m p 68-69{ }^{\circ} \mathrm{C}$ and $[\alpha]_{D}^{25}$ $-116.4^{\circ}$ (in acetone), but, after it had been kept for several months at room temperature, the $m$ p was $56-58{ }^{\circ} \mathrm{C}$. This change has been observed before [39], and cited as evidence for a supposed rearrangement of crystalline $\underset{\sim}{4}$ a to crystalline $1,2: 3,4$-di-0-isopropylidene- $\beta$-D-psicofuranose ( $\underset{\sim}{8}$ ); however, we found that the change in $\mathrm{m} p$ of $\underset{\sim}{4 \mathrm{a}}$ was unaccompanied by any change in $R_{F}$ value, i.r. spectrum (in solution), or optical rotation whereas compound 8 has [32] m p $57-58.5{ }^{\circ} \mathrm{C}$ and $[\alpha]_{D}^{20}-98.2^{\circ}$ (in acetone). Hence, the change in $\mathrm{m} \mathrm{p}$ of $4 \mathrm{a}$ must be ascribable to physical change from an unstable to a stable crystal modification, and not to a chemical change in structure.

Consequently, for the preparation of pure D-psicose $(\underset{\sim}{5} \underset{\sim}{a})$ via oxidation of $1,2: 4,5-d i-0$-isopropylidene-B-Dfructopyranose $(\underset{\sim}{2 a})$, the latter must be free from its $2,3: 4,5$ isomer $(\underset{\sim}{6}$ ) , which is oxidized to the corresponding aldosulose acetal. Pure 1,2:4,5-di-O-isopropylidene- $\beta-$ D-erythro-2,3-hexodiulo-2,6-pyranose ( $\underset{\sim}{3}$ ) undergoes stereospecific reduction with sodium borohydride to give only $1,2: 4,5$-di-o-isopropylidene- $\beta$-D-ribo-hexulopyranose ( $\underset{\sim}{\text { a }}$ ), which exists as two different crystal modifications. Compounds $\underset{\sim}{3}$ and $\underset{\sim}{4}$ a have been characterized, and discrepancies in the literature have been explained.

These results have been incorporated in an article that has been accepted for publication [46].

C. Model Parameters for the Analysis of Skew Conformations by P.m.r. Spectroscopy

1. Introduction

(B. Coxon)

Although the relative importance of chair and nonchair conformations is of considerable interest in the conformational analysis of carbohydrate molecules, there is a scarcity of p.m.r. data for genuine, or widely 
accepted, examples of nonchair conformations [47], especially as several of the earlier assignments of a skew conformation to carbohydrates have been questioned or withdrawn. In order to determine the magnitudes of some of the protonresonance parameters that may be expected to be characteristic of the skew conformations of pyranose compounds, the p.m.r. spectrum (at $100 \mathrm{MHz}$ ) of 3-0-benzoyl$1,2,4-0$-benzylidyne- $\alpha$-D-ribopyranose ( $\underset{\sim}{(})$ has been analyzed by iterative, computer methods. This rigid system provides a suitable model for the testing of theoretical and empirical equations that relate various types of coupling constant with dihedral angle.

\section{Discussion}

Inspection of molecular models of $\underset{\sim}{l}$ reveals that the molecule is rigidly locked in the conformation $\underset{\sim}{\text { la by the }}$ three-point attachment of the benzylidyne group.

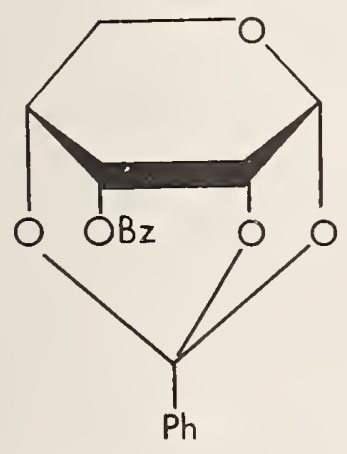

1

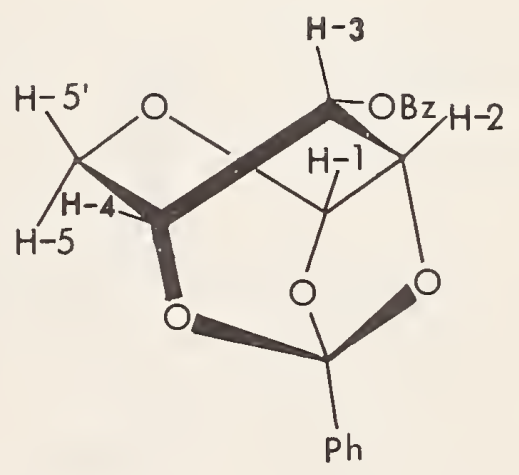

$1 a$

The assignments of the signals of the ring protons in the spectrum (see fig. 3a) of $\underset{\sim}{l}$ in chloroform- $\underline{d}$ were confirmed by noting solvent shifts, and by double-resonance experiments. Some of the double-resonance spectra are shown in fig. 3 ( $c$ and $d$ ). 

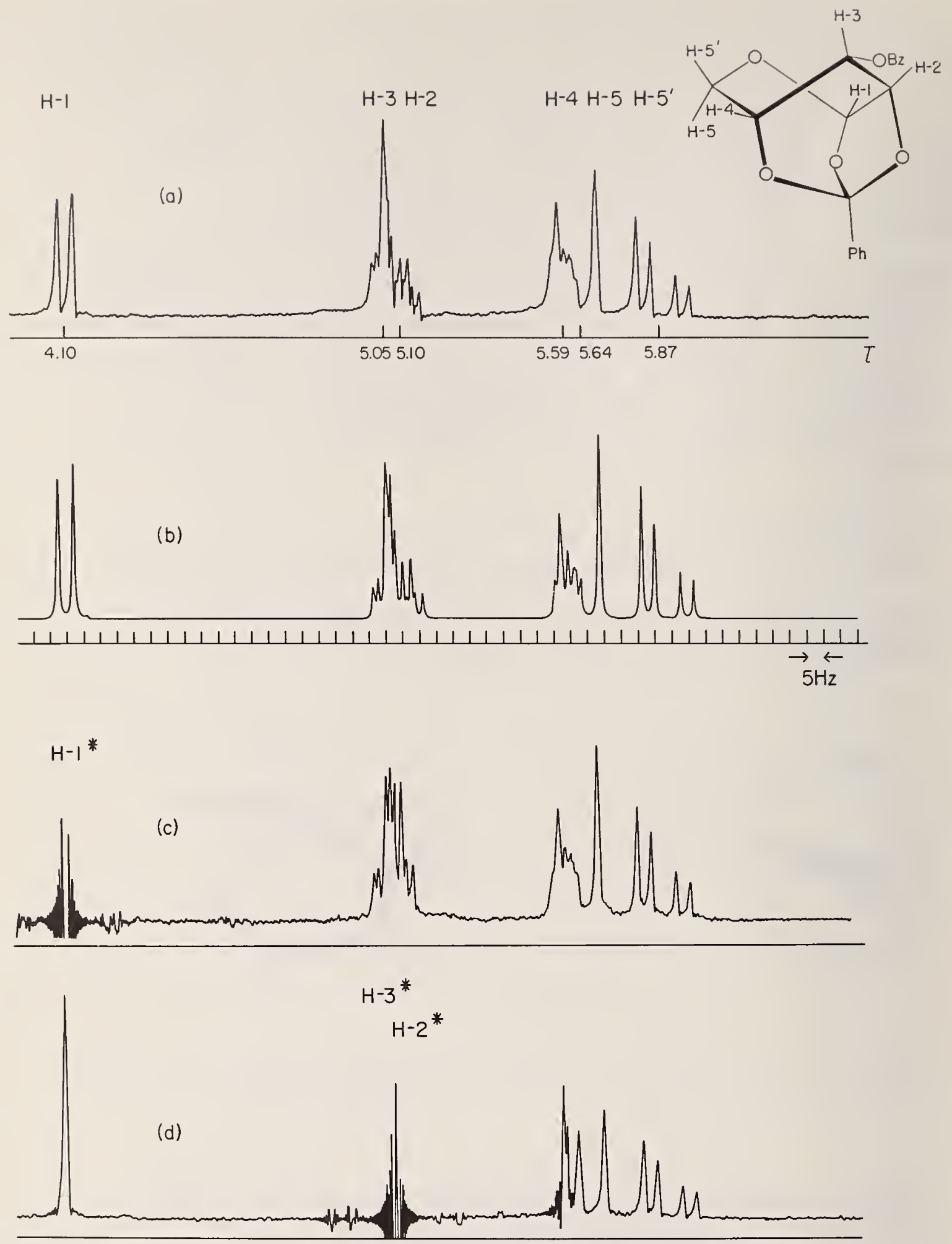

Figure 3. P.m.r. spectra of 1 at $100 \mathrm{MHz}$; the signals of the aromatic protons are not shõwn. (a) Single resonance spectrum, (b) theoretical spectrum from iterative analysis, (c) irradiation of $\mathrm{H}-1$, and (d) irradiation of $\mathrm{H}-2$ and $\mathrm{H}-3$. 
Initial parameters for the iterative analysis of the spectra were derived by $A B X$ analysis of the $\mathrm{H}-3, \mathrm{H}-2$, and H-4 nuclei with $\mathrm{H}-\mathrm{l}$ decoupled ( $\mathrm{fig}$. 3c), and by ABX analysis of the $\mathrm{H}-5, \mathrm{H}-5^{\prime}$, and $\mathrm{H}-4$ signals in the single-resonance spectrum ( $f i g .3 a$ ); $H-5$ resonates at lower field than $H-5^{\prime}$. With $\mathrm{H}-1$ decoupled, $\mathrm{H}-2$ still resonated as a quartet (fig. 3c) whose spacings suggested long-range coupling with H-4. This situation was confirmed by irradiation of $\mathrm{H}-4$. Good agreement was obtained between the observed spectrum ( $f i g .3 a$ ) and the theoretical spectrum (fig. 3b) calculated from the parameters obtained after 13 iterations.

For the particular values of proton-proton dihedral angles $(\phi)$ present in $\underset{\sim}{\operatorname{la}}$, the corresponding values of the vicinal coupling-constan's may be used to test the applicability of the Karplus equation,

$$
\phi=\cos ^{-1}\left\{\frac{0.5 \pm\left[0.25+36(J+0.28]^{1 / 2}\right.}{18}\right\}
$$

to carbohydrate systems. The form [48] of this equation is convenient, in that solution of the quadratic equation in $\cos \phi$ provides two roots, one of which corresponds to $0^{\circ} \leqslant \phi \leqslant 88^{\circ}$, and the other, to $88^{\circ} \leqslant \phi \leqslant 180^{\circ}$, with the use of the same constants for each type of angle.

The vicinal-proton, dihedral angles obtained by measurement of Dreiding molecular models of la, and the two angles that were calculated from each vicina ̃̃-coupling constant by using equation ( 1 ), have been correlated with the angles that would be expected for an idealized, symmetrical skew form [49] of the pyranoid ring.

In a molecular model of $\underset{\sim}{\text { la }}$, the conformation is somewhat distorted from the idealized skew form, as only the corresponding values of $\phi_{3,4}$ were in close agreement therewith. The values of $\phi_{2,3}, \phi_{3,4}$, and $\phi_{4,5}$ calculated from equation (l) agree quite well with the values estimated 


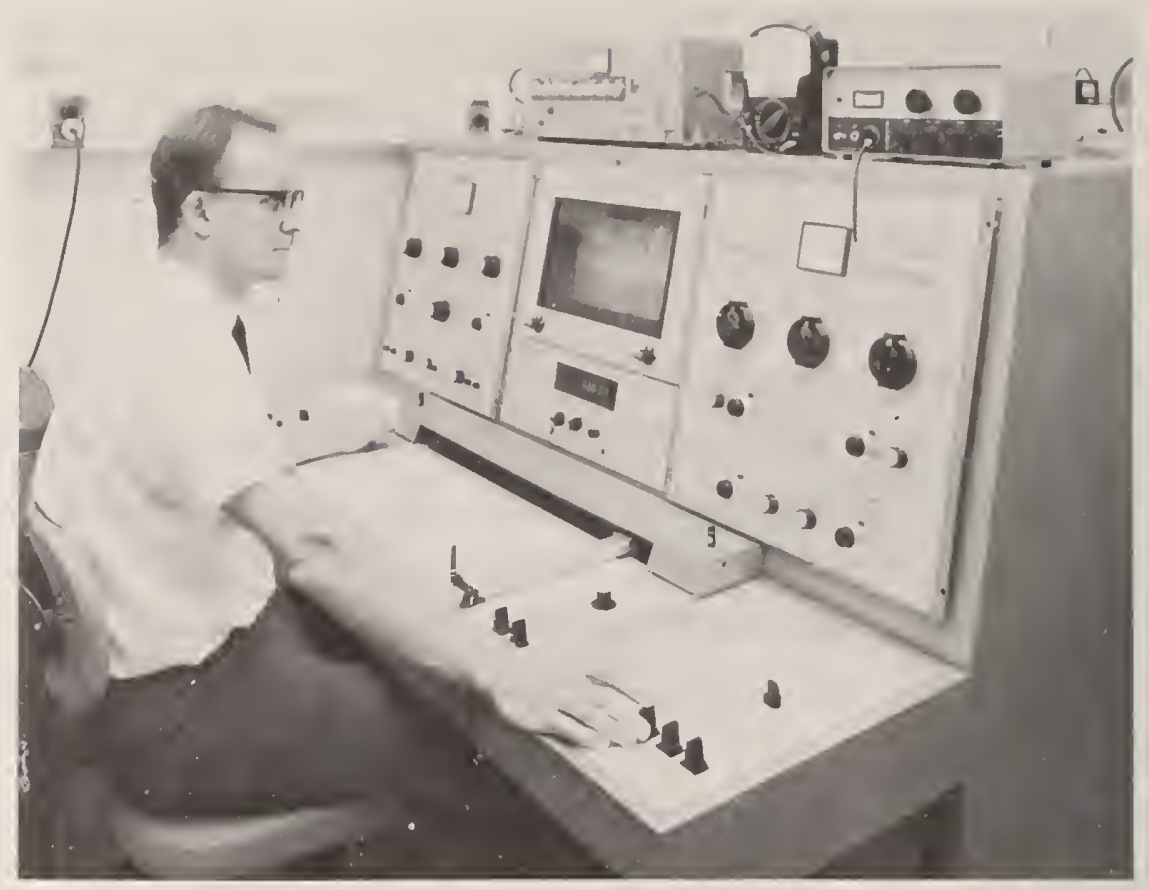

Figure 4. Adjustment of the strength of the magnetic field, at the console of a nuclear magnetic resonance spectrometer.

by study of molecular models, but the agreement is less good for $\phi_{1,2}$ and $\phi_{4,5}$.

The value of the geminal coupling $\left(J_{5}, 5,11.7 \mathrm{~Hz}\right)$ of $\frac{1}{\sim}$ is intermediate between those (10.8-10.9 and $13.4-14.0 \mathrm{~Hz})$ found [50] for $\alpha$ anomers or $\beta$ anomers of D-ribopyranose tetrabenzoate or of the related l-halides, for which a dependence on the orientation of the vicinal, electronegative substituent (benzoyloxy) on $\mathrm{C}-4$ has been indicated [50]. Although the value of $11.7 \mathrm{~Hz}$ for $J_{5,5}$, is closer to those of the a anomers, the orientation of $\mathrm{O}-4$ with respect to $\mathrm{H}-5^{\prime}$ and $\mathrm{H}-5$ more nearly resembles the trans-gauche arrangements found $[50]$ in the $\beta$ anomers. 


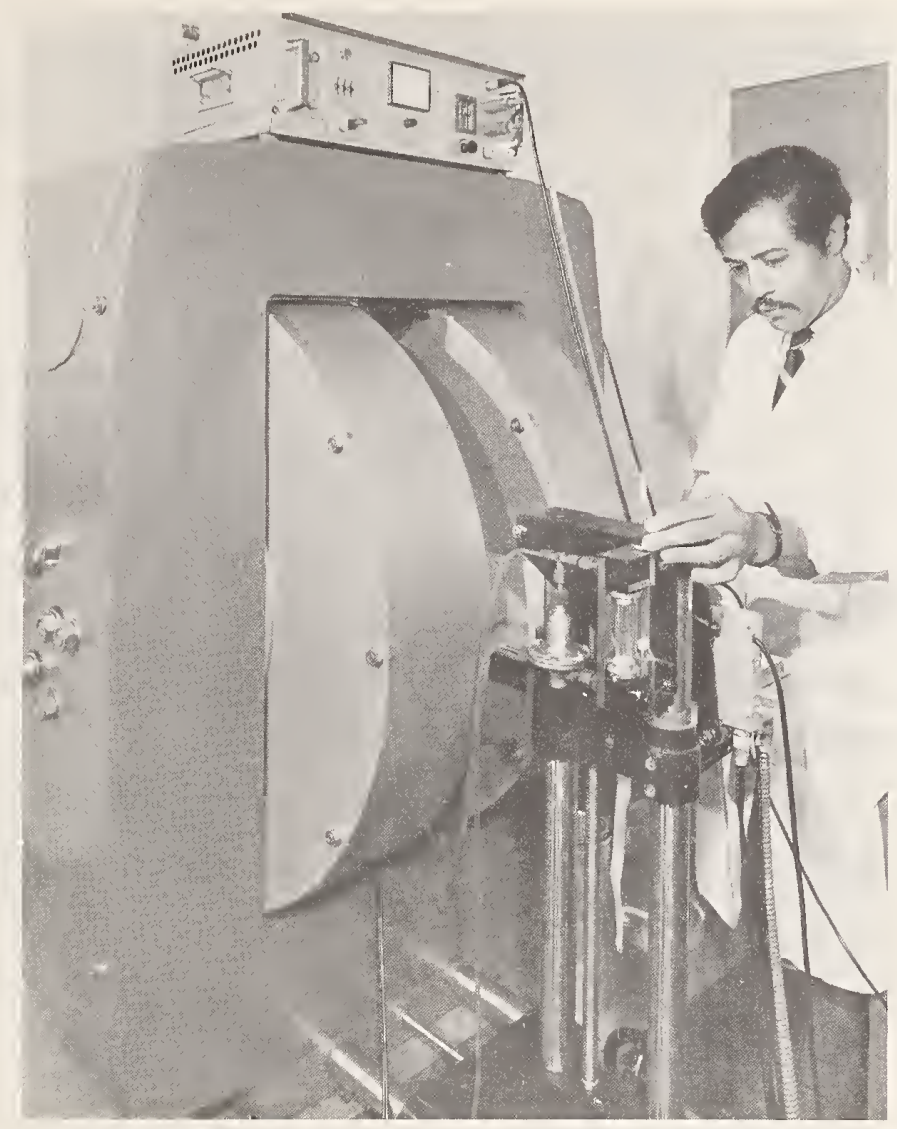

Figure 5. Positioning a sample tube, prior to insertion into the probe head of a nuclear magnetic resonance spectrometer.

More-detailed considerations suggest that these results can be interpreted in terms of the proximity of $\sigma$ and $p$ orbitals of the $\mathrm{H}-\mathrm{C}-5$ bonds and the ring oxygen-atom, respectively, which can lead to a $p \rightarrow \sigma$ electron transfer that results in a positive increment to the geminal coupling-constant, in addition to that due to o-electron withdrawal by a suitably disposed, electronegative substituent.

The long-range coupling $J_{2}, 4$ of $\underset{\sim}{l}$, amounting to $2.45 \mathrm{~Hz}$, is probably the largest thus far observed over four saturated bonds of a carbohydrate system. In the skew conformation $\underset{\sim}{\mathrm{l} a}$, the dihedral angles $\phi^{\prime}$ ( of $\mathrm{H}-2$ and $\mathrm{C}-4$, 
about the $\mathrm{C}-2-\mathrm{C}-3$ bond) and $\phi^{\prime \prime \prime}$ ( of $\mathrm{H}-4$ and $\mathrm{C}-2$, about the C-3--C-4 bond) are $\sim 170^{\circ}$ and $\sim 171^{\circ}$, respectively, and, therefore, the orientation of $\mathrm{H}-2, \mathrm{C}-2, \mathrm{C}-3, \mathrm{C}-4$, and $\mathrm{H}-4$ is very close to the planar W-arrangement that is considered [51,52] to be optimal for coupling ( ${ }^{4} J$ ) over four saturated bonds. In $\underset{\sim}{\mathrm{l} a}, \mathrm{H}-2$ and $\mathrm{H}-4$ have quasi and equatorial orientations with respect to the plane of the pyranoid ring.

The positive sign of $J_{2,4}$ is in agreement with the indirect, through-bond mechanism proposed [53] by Barfield. However, Barfield's mechanism [53] does not satisfactorily account for the reported [54] variation of the sign of ${ }^{4} \mathrm{~J}$ with the equatorial--equatorial or equatorial---axial orientations of ring protons of the chair conformations of pyranoses. Barfield's theory [53] has been interpreted [55] in terms of the equation

$$
{ }^{4} J=0.7\left(\cos ^{2} \phi^{\prime}+\cos ^{2} \phi^{\prime \prime \prime}\right)-0.3 .
$$

Substitution in this equation of the values of $\phi^{\prime}$ and $\phi^{\prime \prime}{ }^{\prime}$ for $\operatorname{la}_{\sim}$ gives for ${ }^{4} J$ a value of $1.06 \mathrm{~Hz}$ that does not agree with the value of $J_{2}, 4$ observed, namely, $2.45 \mathrm{~Hz}$. Such disagreement has been noted [55] for ${ }^{4} J$ in a series of tetrachlorobicyclo[2,2,1]heptane derivatives, and it has been suggested [55] that better agreement with experiment could be obtained by use of the equation

$$
{ }^{4} J=A \cos ^{2} \phi^{\prime} \cos ^{2} \phi^{\prime \prime \prime}-B \text {, }
$$

where $B=0.35$, and $A=0.31$ for $0^{\circ} \leqslant \phi^{\prime}, \phi^{\prime \prime}{ }^{\prime} \leqslant 90^{\circ}, 1.07$ for $0^{\circ} \leqslant \phi^{\prime} \leqslant 90^{\circ} \leqslant \phi^{\prime \prime} \prime \leqslant 180^{\circ}$, or 3.61 for $90^{\circ} \leqslant \phi^{\prime}, \phi^{\prime \prime}$ $\leqslant 180^{\circ}$. For ${\underset{\sim}{\sim}}_{\sim}$, equation (3) yields ${ }^{4} \mathrm{~J} 3.07 \mathrm{~Hz}$, which agrees more closely with the value observed for $J_{2,4^{\circ}}$

These results were incorporated in an article that has been published [56]. 
D. The Conformations of $1,2: 4,6-d i-0$-benzylidene- $\alpha-D-$ giucopyranose Derivatives

(B. Coxon)

\section{Introduction}

There is little doubt that the favored conformation of

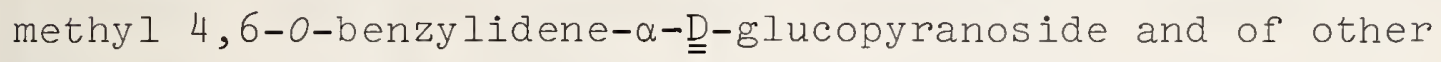
similar derivatives having the same configuration at C-4 is the $C 1$ (D) form. However, there has been controversy concerning the possible chair or skew conformations of

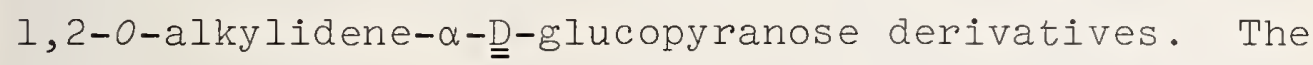
investigation of derivatives containing both 1,2- and 4,6-cyclic acetal substituents was, therefore, of interest, and $1,2: 4,6-d i-0-b e n z y l i d e n e-\alpha-\underline{D}-g l u c o p y r a n o s e ~(\underset{\sim}{1})$ and the 3-acetate ( $\underset{\sim}{2}), 3$-benzoate $(\underset{\sim}{3})$, and 3-methanesulfonate ( $\left.\sim_{\sim}\right)$ prepared by Fletcher and co-workers [57] have been found to be suitable for study by p.m.r. spectroscopy at $100 \mathrm{MHz}$.

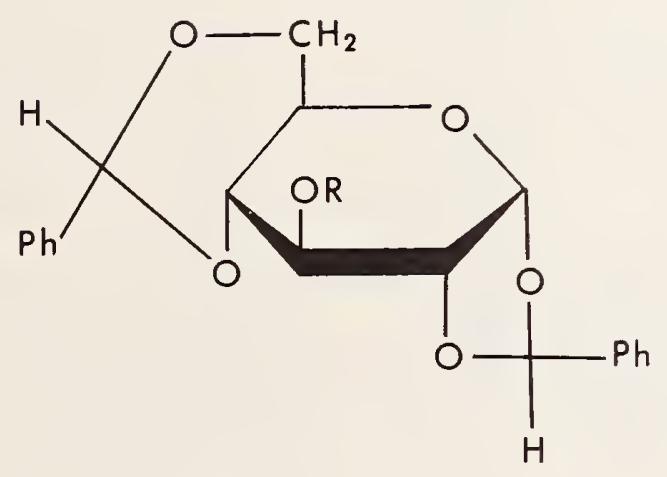
$1 R=H$
$2 R=A C$
$3 R=B z$
$4 R=M s$

2. Discussion

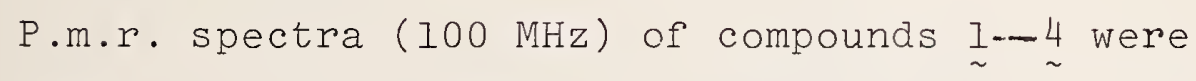
recorded for their solutions in chloroform- $\underline{a}$ or pyridine, 
or in mixtures thereof. Although parts of the spectra were complex, because of overlap of multiplets, the signal multiplicities observed for the protons on the sugar ring were only those to be expected from coupling over two, or three, bonds. The spectral assignments for these protons were confirmed by spin-decoupling and spin-tickling, and by solvent shifts. Comparison of the chemical shifts of the benzylic protons with those of the O-benzylidene- $\alpha-$ Dglucopyranoses and 4,6-o-benzylidene- $\alpha$-D-glucopyranosides indicated that, for each compound, the singlet at low field could be assigned to the 1,2 benzylic proton, and that at higher field, to the 4,6 benzylic proton.

The chemical shifts of the protons attached to the sugar ring of $\underset{\sim}{1-}-\underline{\sim}$ were sufficiently different that their spectra could readily be analyzed by first-order methods. However, an estimate of the accuracy of the parameters so derived, and of the validity of the analysis, was obtained by iterative analysis [58] of the spectrum (see fig. 6a) of compound $\underset{\sim}{4}$ a.s a seven-spin system $(\mathrm{H}-\mathrm{I}-\mathrm{H}-5, \mathrm{H}-6 e$, and $\mathrm{H}-6 \alpha)$. From spin-tickling experiments on 2 , it was established that the sign of $J_{6 e}, 6 a$ is the opposite of that of $J 5,6 e$ and $J 5,6 a$, and, therefore, $J 6 e, 6 a$ was made negative in the iterative analysis of the spectrum of $\underset{\sim}{4}$. Support for the validity of the assignments was provided by the good agreement between the theoretical spectrum ( $f i g .6 b$ ) that had been computed from the refined parameters and the observed spectrum (fig. 6a), and by the small error-parameters obtained. From the coupling-constant data for 4, it was calculated that the maximum differences between the first-order value and the refined value of each coupling constant is about $13 \%$ of the latter, and that the corresponding mean difference for seven coupling-constants is $6 \%$. 


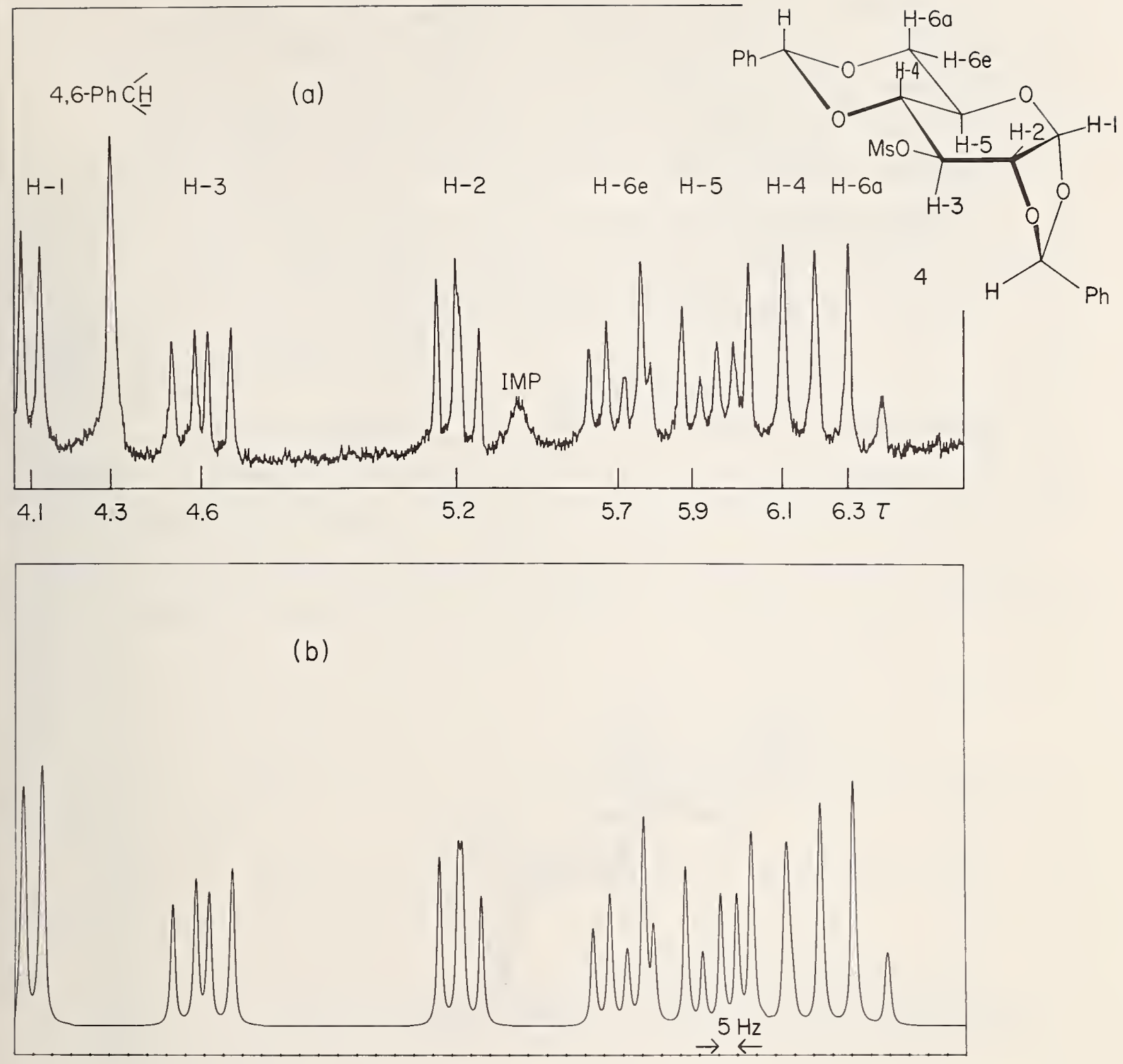

Figure 6. Partial p.m.r. spectra of 4. (a) Observed spectrum of solution in pyr̃idine at $100 \mathrm{MHz}$, and ( $b$ ) computed spectrum after iterative analysis as a seven-spin system. 
The values of $J_{3,4}, J_{4}, 5$, and $J_{5,6}$ shown by $\underset{\sim}{1-}-\underset{\sim}{4}$ are appropriate for pairs of protons having a trans-diaxial orientation, and the medium-sized values of $J 5,6$ e observed are typical of protons having a gauche-axial--.equatorial orientation. However, the values of $J_{1,2}$ are consistently $l \mathrm{~Hz}$ larger, and the values of $J_{2,3}, \sim 3.6 \mathrm{~Hz}$ smaller, than those of methyl 4,6-o-benzylidene-a-D-glucopyranoside, indicating that the proton--proton dihedral angles $\phi_{1,2}$ and $\phi_{2,3}$ of $\underset{\sim}{1-}-\underset{\sim}{4}$ are smaller than those of the $\alpha-\underline{D}$-glucopyranoside. Application of the refined Karplus equation [59] to compounds $\underset{\sim}{1-L_{2}}$ gave the approximate values $\phi_{1,2} 38-39^{\circ}$ and $\phi_{2,3}$ $142-145^{\circ}$, which compare favorably with the values of $25^{\circ}$ and $135^{\circ}$, respectively, measured on Dreiding molecular models.

The results are consistent with the conformation depicted in formula $\underset{\sim}{5}$, in which the m-dioxane ring adopts a chair

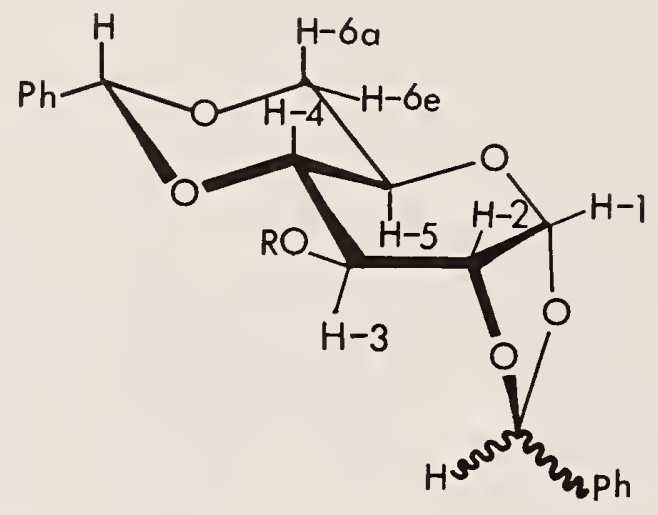

5

shape, and in which the pyranoid ring has a flattenedchair shape (because of attachment of the 1,2-0-benzylidene group). As compound $\underset{\sim}{I}$ had been prepared [57] under acidic conditions, it is assumed that the phenyl group of the 4,6-0-benzylidene substituent is equatorially attached to the chair shape of the $m$-dioxane ring $[60,61]$. 
Although study of molecular models revealed that the cyclic acetal substituents would not sterically prohibit the adoption of a skew shape by the pyranoid ring of the dibenzylidene derivatives (were this energetically feasible), only a small value of $J_{2,4}(0.03 \mathrm{~Hz})$ was obtained from the iterative analysis of the spectrum of compound $\underset{\sim}{4}$. Hence, there is no evidence for any conformational equilibrium involving that skew shape of the pyranoid ring in which $\mathrm{H}-2$ and $\mathrm{H}-4$ and their intervening carbon atoms have an almost planar W-arrangement [52].

In conclusion, the favored conformation of 1,2:4,6-di-

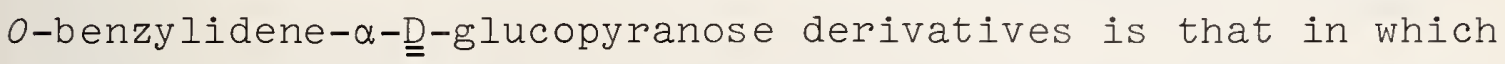
the pyranoid ring has a flattened-chair shape. Nevertheless, as the coupling constants $J_{2,3}$ and $J_{3,4}$ of these derivatives are, for all of the compounds studied, significantly larger than those of 1,2-0-alkylidene- and -arylidene- $\alpha-\underline{\underline{D}}-$ glucopyranoses, there is evidently less conformational distortion of the di- than of the mono-benzylidene acetals. Thj.s situation may be ascribed to the tendency of the 4,6-0-benzylidene substituent to increase the rigidity of the pyranoid ring.

Numerical data are given in an article that has been accepted for publication [62].

E. Iterative Analysis of the Proton Magnetic Resonance Spectra of $1,2: 3,5-D i-0$-isopropylidene- $\alpha-$-glucofuranose Derivatives

(B. Coxon)

1. Introduction

In continuation of previous work $[48,63,64]$ on the conformations of $1,2: 3,5$-di-o-arylidene- and -alkylidene- $\alpha-$ D-glucofuranose derivatives, an investigation by p.m.r. spectroscopy of the conformations of $1,2: 3,5-d i-0-$ isopropylidene- $\alpha-$ D-glucofuranose derivatives has been started. A necessary preliminary to the assignment of conformation 
by this technique is analysis of the p.m.r. spectra, which, depending on the relative magnitudes of the chemical shifts and coupling constants may be simple or complex. Analysis of complex spectra is assisted considerably by the availability of spectrometers that operate at a high strength of magnetic field (>20 T), and by use of computer programs that (a) calculate a trial, theoretical spectrum and then (b) refine, with the input of suitably assigned, experimental transition-frequencies, the initial parameters until the theoretical spectrum and the experimental transitions converge to a "best" fit according to a criterion of least squares.

In the present report are described the chemical shifts and coupling constants obtained by iterative analysis of (a) 6-deoxy-1,2:3,5-di-o-isopropylidene-6-phthalimido- $\alpha$-Dglucofuranose $(\underset{\sim}{)}$ ), (b) 6-deoxy-1,2:3,5-di-o-isopropylidene-6-

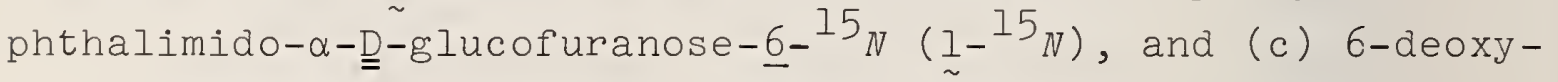
$1,2: 3,5-d i-0$-isopropylidene- $\alpha-\underline{\underline{D}}-\mathrm{glucofuranose}-d_{12}\left(\underset{\sim}{2-d_{12}}\right)$.

2. Results and Discussion

The synthesis of compounds $\underset{\sim}{I}$ and $\underset{\sim}{1}-{ }^{15} N$ has been described previously $[65,66]$. At a radiofrequency of $100 \mathrm{MHz}$, their p.m.r. spectra in several different solvents had resisted analysis, because of overlapping of multiplets. However, at a frequency of $220 \mathrm{MHz}$ (see figs. $7 \mathrm{a}$ and $7 \mathrm{~b}$ ), these multiplets are sufficiently well separated for a set of trial parameters to be obtainable by simple, first-order analysis.

The p.m.r. spectrum of compound $\underset{\sim}{1}$ was analyzed by means of the LAOCN3 program [67] as a seven-spin system consisting of $\mathrm{H}-1, \mathrm{H}-2, \mathrm{H}-3, \mathrm{H}-4, \mathrm{H}-5, \mathrm{H}-6$, and $\mathrm{H}-6^{\prime}$. In the iterative part of the calculation, 66 theoretical transitions were assigned to 21 experimental line-positions, and variation of seven chemical shifts and of the coupling constants 

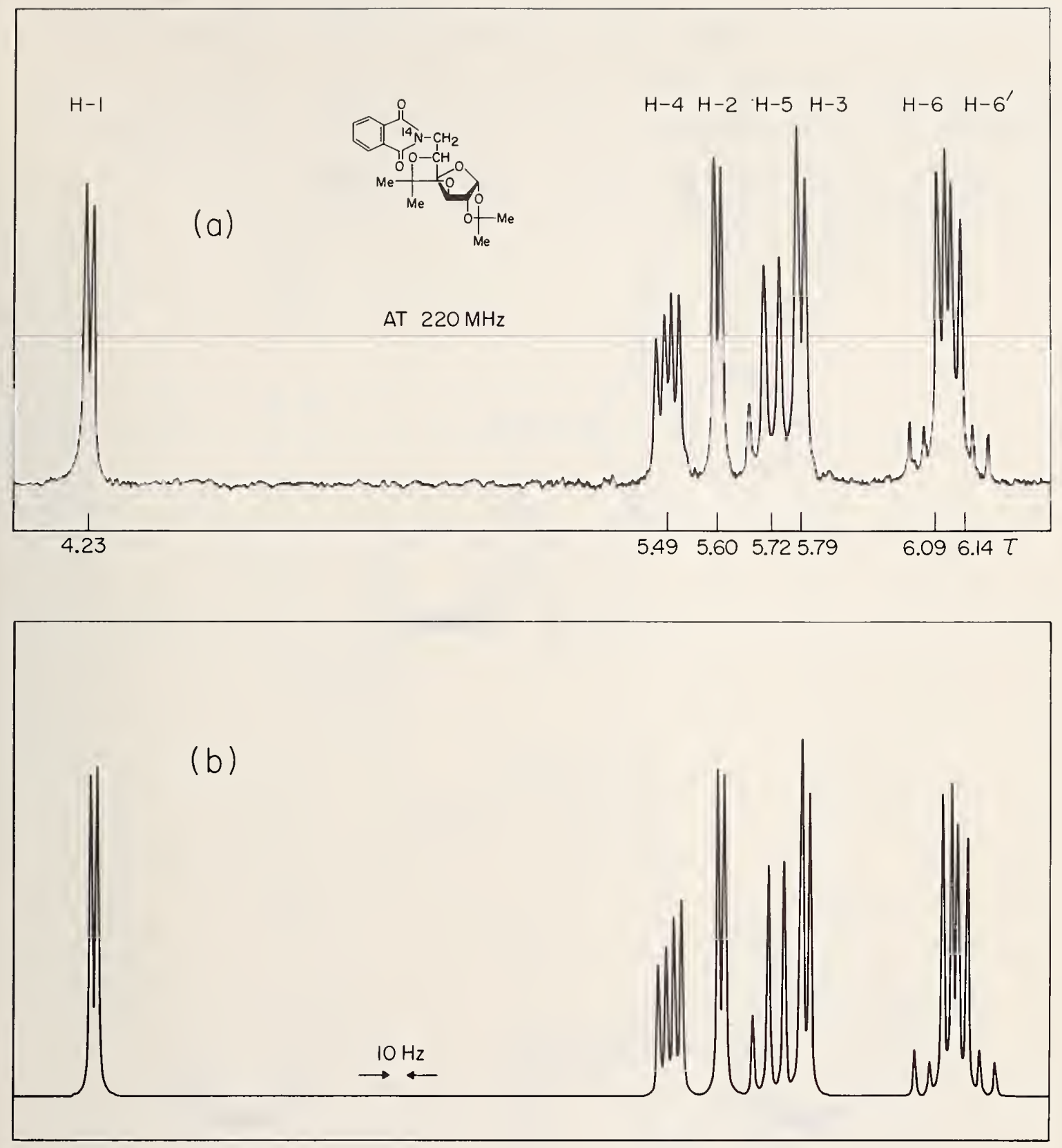

Figure 7. Partial p.m.r. spectra of 7 . (a) Observed spectrum at $220 \mathrm{MHz}$, and ( $\tilde{b})$ computed spectrum after iterative analysis as a seven-spin system. 
$J_{1,2}, J_{2,3}, J_{3,4}, J_{4,5}, J_{5,6}, J_{5,6}$, and $J_{6,6}$, was permitted. A good fit of experimental and theoretical spectra (see figs. $7 \mathrm{a}$ and $7 \mathrm{~b}$ ) was obtained after only one iteration. The refined coupling-constants and chemical shifts are shown in tables 24 and 25, respectively, together with the corresponding parameters of compounds $\underset{\sim}{1}-15 N$ and $\underset{\sim}{2}$.

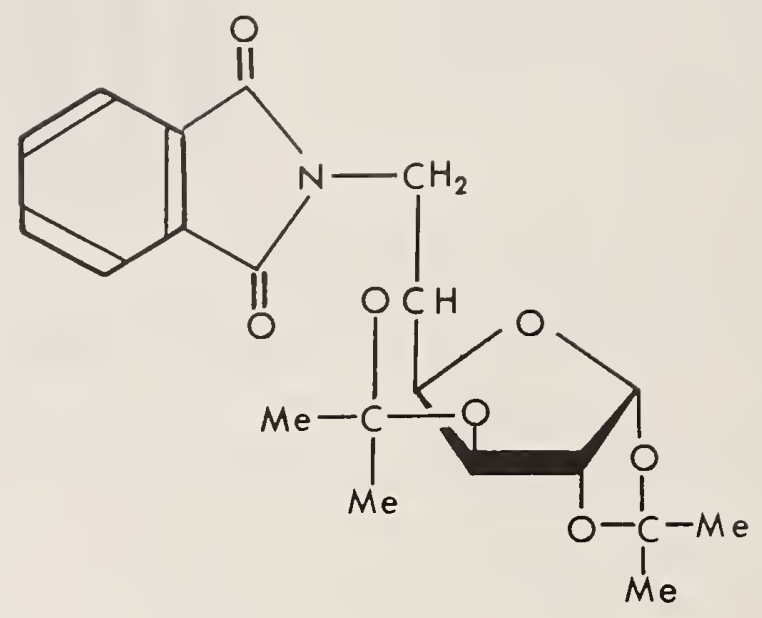

$$
\begin{aligned}
& 1\left(N={ }^{14} N\right) \\
& 1-{ }^{15} N\left(N={ }^{15} N\right)
\end{aligned}
$$

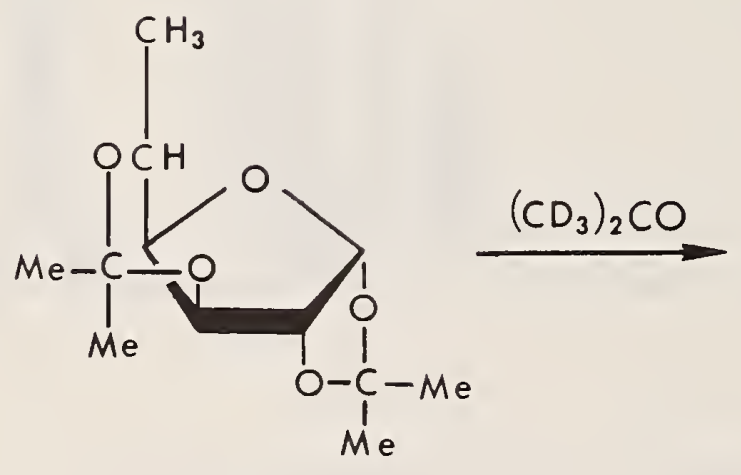

2

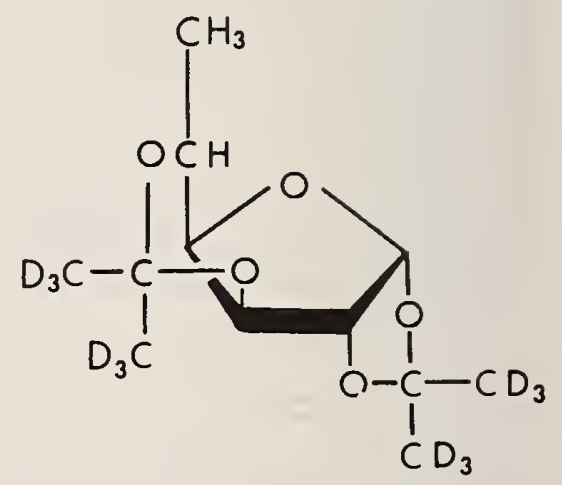

$2-d_{12}$ 

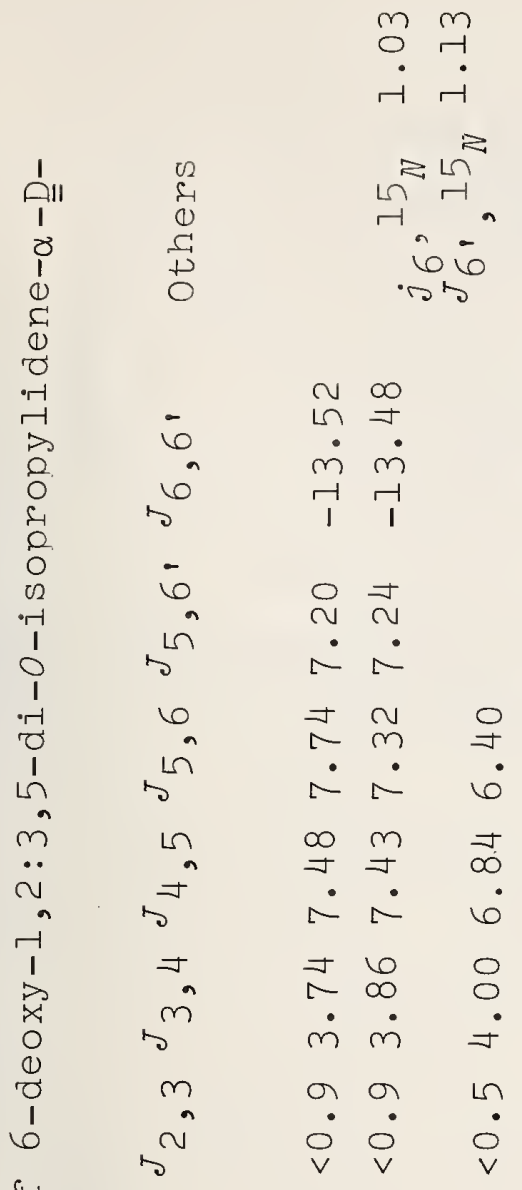

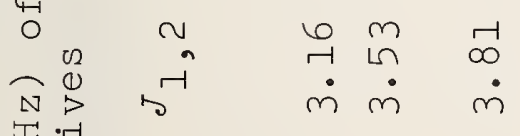

西㔔

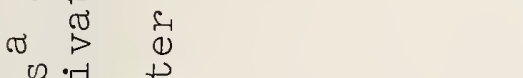

$\begin{array}{llll}1 & \varepsilon & 0 & 0 \\ \text { E } & 0 & 0\end{array}$

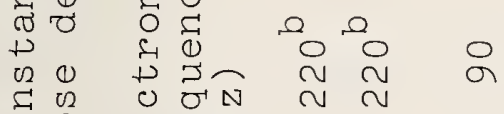

O 0 (1) 0 霍

$0 \underset{1}{0}$ 饮点

80 s

.

,

उ.

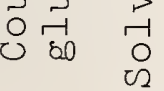

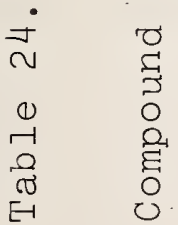

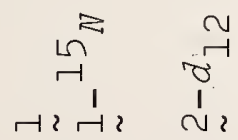

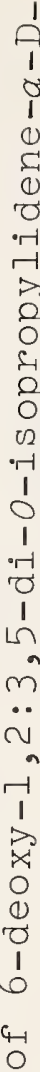

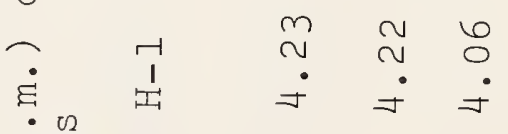

Q 0

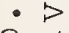

ह.न हा

$\stackrel{+}{\square} \stackrel{0}{\downarrow} 0$

$\leftarrow>\quad 0$

C. E

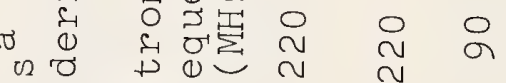
(1) 0 \&

$\begin{array}{llll}4 & 11 & 0 & 4 \\ -1 & 02 & 0\end{array}$

त् 0 थ

०त

os $ᄃ$

고엄

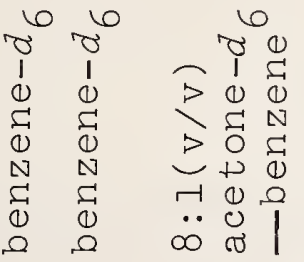

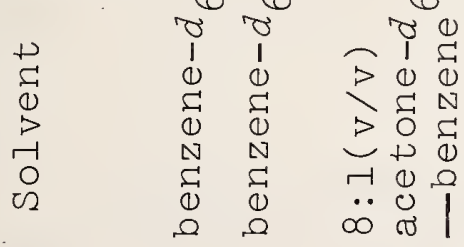

ช

$\underset{\sim}{\mathscr{\sigma}} \stackrel{\infty}{\infty}$

$\begin{array}{lll}0 & 4-1 \\ 0 & 4 & 0 \\ 0 & 0 & 0\end{array}$

-1
$\rightarrow>$

क ज

(1)

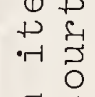

हु 0

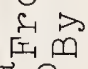

ส

ชิ ริ

0 ㄸ

घ.

ङ

2
1
1
0
0
0
0
0

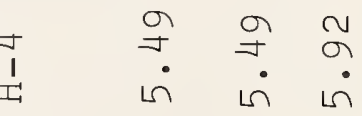

$\begin{array}{llll}m & a & \infty & m \\ 1 & \sim & \sim & \infty \\ & \dot{\pi} & \dot{\kappa} & \dot{\kappa}\end{array}$

空

$\begin{array}{ccc}0 & 0 & 6 \\ \bullet & 0 & \dot{7} \\ \dot{1} & \dot{1}\end{array}$

$\stackrel{\infty}{\sim}$

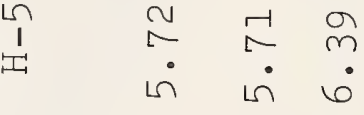

N

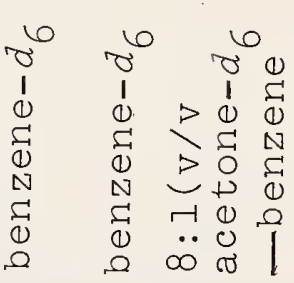

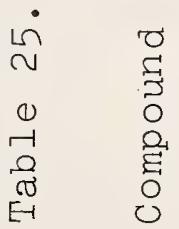

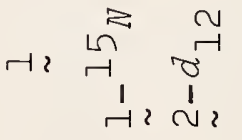

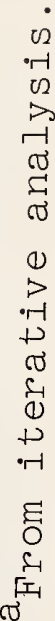


For compound ${ }_{\sim}^{1-}{ }^{15} \mathrm{~N}$, the introduction of a coupled ${ }^{15} \mathrm{~N}$ nucleus gave eight nonequivalent nuclei, each with spin $1 / 2$, thus exceeding the capabilities of the LAOCN3 program. Fortunately, however, $\mathrm{H}-2$ and $\mathrm{H}-3$ are only very weakly coupled, and so the eight-spin system was divided into ( $a$ ) a six-spin sub-system consisting of ${ }^{15} \mathrm{~N}, \mathrm{H}-3, \mathrm{H}-4, \mathrm{H}-5, \mathrm{H}-6$, and $H-6^{\prime}$, and $(b)$ a two-spin system consisting of $\mathrm{H}-1$ and $\mathrm{H}-2$. Each of these sub-systems was then analyzed separately. For the iterative analysis of the six-spin sub-system, 60 theoretical transitions were assigned to 26 experimental lines, and variation of the proton chemical-shifts and the coupling constants $J_{3,4}, J_{4}, 5, J_{5}, 6, J_{5,6}, J_{6,6}, J_{6},{ }^{15}{ }_{N}$, and $J_{6},{ }^{15}{ }_{N}$ was allowed, but not of the ${ }^{15} \mathrm{~N}$ chemical-shift, for which no experimental data are as yet available (for the purposes of the calculation, this shift was assigned an arbitrary, fixed value). Iteration proceeded for four cycles, with r.m.s. errors of the line positions of $0.775,0.183,0.157$, and 0.157 . For the iterative analysis of the trivial, two-spin sub-system, the four theoretical transitions were assigned to the four observed lines of the $\mathrm{H}-1$ and $\mathrm{H}-2$ doublets, and variation both of chemical shifts and of the coupling constant was permitted. Iteration proceeded for three cycles, with r.m.s. errors of $0.070,0.001$, and 0.001 . The refined parameters are shown in tables 24 and 25, and the agreement between the theoretical spectrum calculated from the refined parameters and the observed spectrum at $220 \mathrm{MHz}$ is depicted in fig. 8. This theoretical spectrum is a composite of those of the twoand six-spin sub-systems.

Compound $\underset{\sim}{2-d_{12}}$ was synthesized by direct exchange of the isopropylidene groups in 6-deoxy-1,2:3,5-di-0-isopropylidene$\alpha-\underline{=}-g l u c o f u r a n o s e(2)$ with acetone- $d_{6}$ in the presence of concentrated sulfuric acid, anhydrous copper(II) sulfate, and Drierite, The dodeca-deuterated derivative was used for these studies because, in the p.m.r. spectrum of the non- 

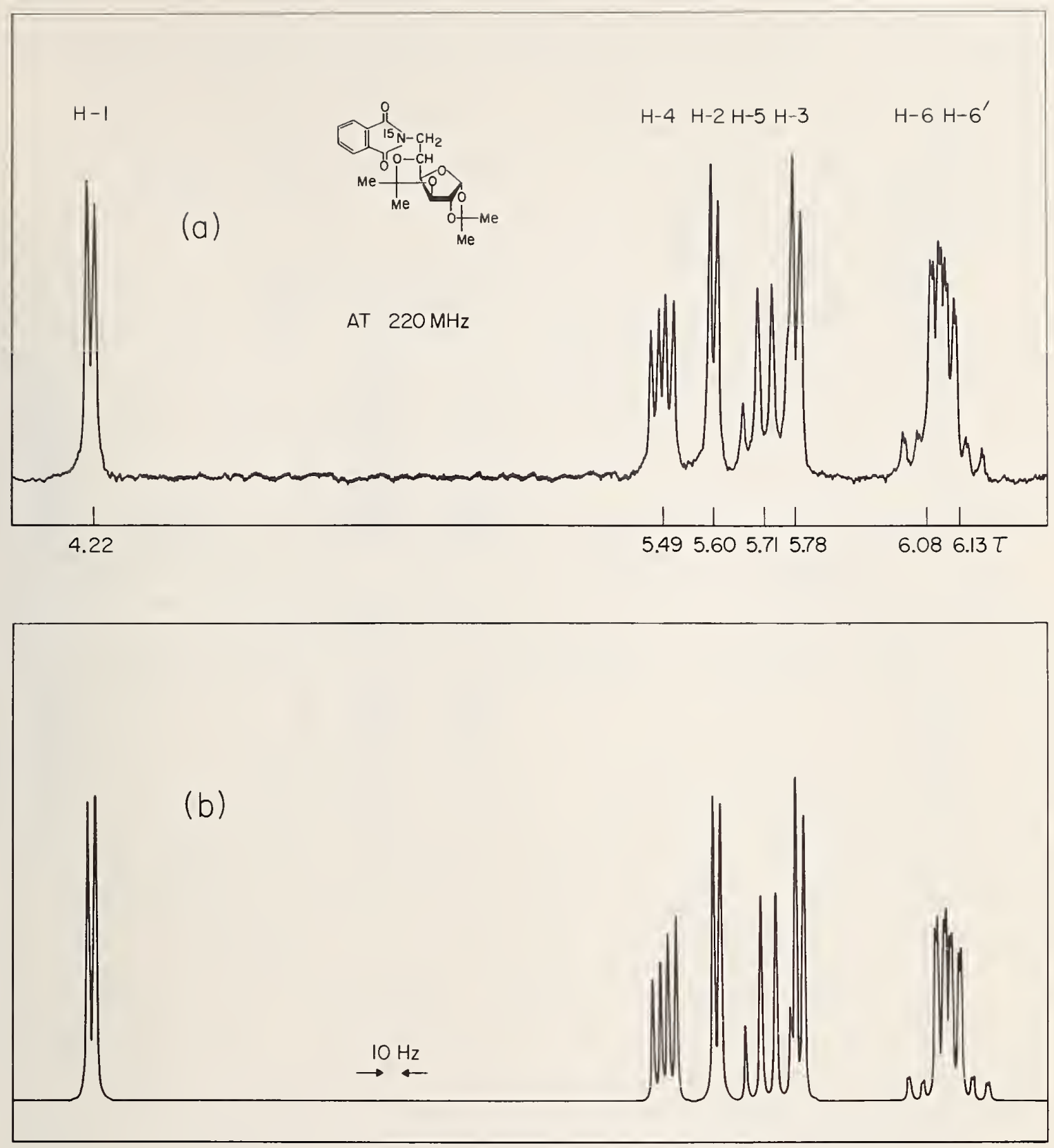

Figure 8. Partial p.m.r. spectra of $1-^{15} \mathrm{~N}$. (a) Observed spectrum of solution in beñzene-d6 at $220 \mathrm{MHz}$, and (b) computed spectrum after iterative analyses of two- and six-spin systems. 
deuterated compound $\underset{\sim}{2}$ at $90 \mathrm{MHz}$, the $\mathrm{H}_{3} \mathrm{C}-6$ signal of interest was obscured by the signals of the isopropylidene methyl groups. The experimental line-positions obtained from $\underset{\sim}{2-d_{12}}$ were average values of four spectra at $90 \mathrm{MHz}$. These were recorded by sweeping twice from low to high frequency, and twice from high to low frequency.

In the spectrum of $2-d_{12}$, the $\mathrm{H}_{3} \mathrm{C}-6$ signal showed as a sharp doublet due to coupling with $\mathrm{H}-5$. This spectrum was analyzed as an $\mathrm{A}_{3} \mathrm{BCDEF}$ system consisting of $\mathrm{H}_{3} \mathrm{C}-6, \mathrm{H}-5, \mathrm{H}-4$, $\mathrm{H}-3, \mathrm{H}-2$, and $\mathrm{H}-\mathrm{l}$ by using a magnetic-equivalence factoringprogram that has recently become available [68] in iterative form (UEAITR). The purpose of this program, for which compound $2-d_{12}$ was designed as a test case, is to use any magnetic equivalence present in the system (such as that for the methyl protons) to factorize the Hamiltonian n.m.r. function, and thereby decrease the size of the corresponding Hamiltonian matrix and, also, the computer time required. In previous analyses [63] of seven-spin systems by use of the LAOCN3 program, it had been found necessary to limit differentiation of the largest Hamiltonian matrixes to the diagonal terms, in order that the iterative calculations should not take an excessive time on the computer.

By using the UEAITR program, computation of a trial, theoretical spectrum for $\underset{\sim}{2-d_{12}}$ took $25 \mathrm{sec}$. Initial inputparameters for the calculation were obtained by simple, first-order analysis of the spectrum, and, indeed, $\mathrm{H}-2$ was not observably coupled to $\mathrm{H}-3$, as in the 6-deoxy-6phthalimido derivatives $\underset{\sim}{1}$ and $1-{ }_{\sim}^{15} N$, and in other $\alpha-\underline{\underline{D}}-$ glucofuranose derivatives $[48,63,64]$. In the iterative computation, 118 theoretical transitions were assigned to 17 experimental lines, and variation of the six chemicalshifts and coupling constants $J_{1,2}, J_{2}, 3, J_{3,4}, J_{4}, 5$, and $J_{5,6}$ was permitted. The experimental and theoretical spectra were in excellent agreement after four iterations (figs. 9b and 9 a, respectively). The r.m.s. errors of these iterations 84 
(a)

$H-1$

$\mathrm{H}-2 \quad \mathrm{H}-3 \mathrm{H}-4 \quad \mathrm{H}-5$

$\mathrm{CH}_{3}$
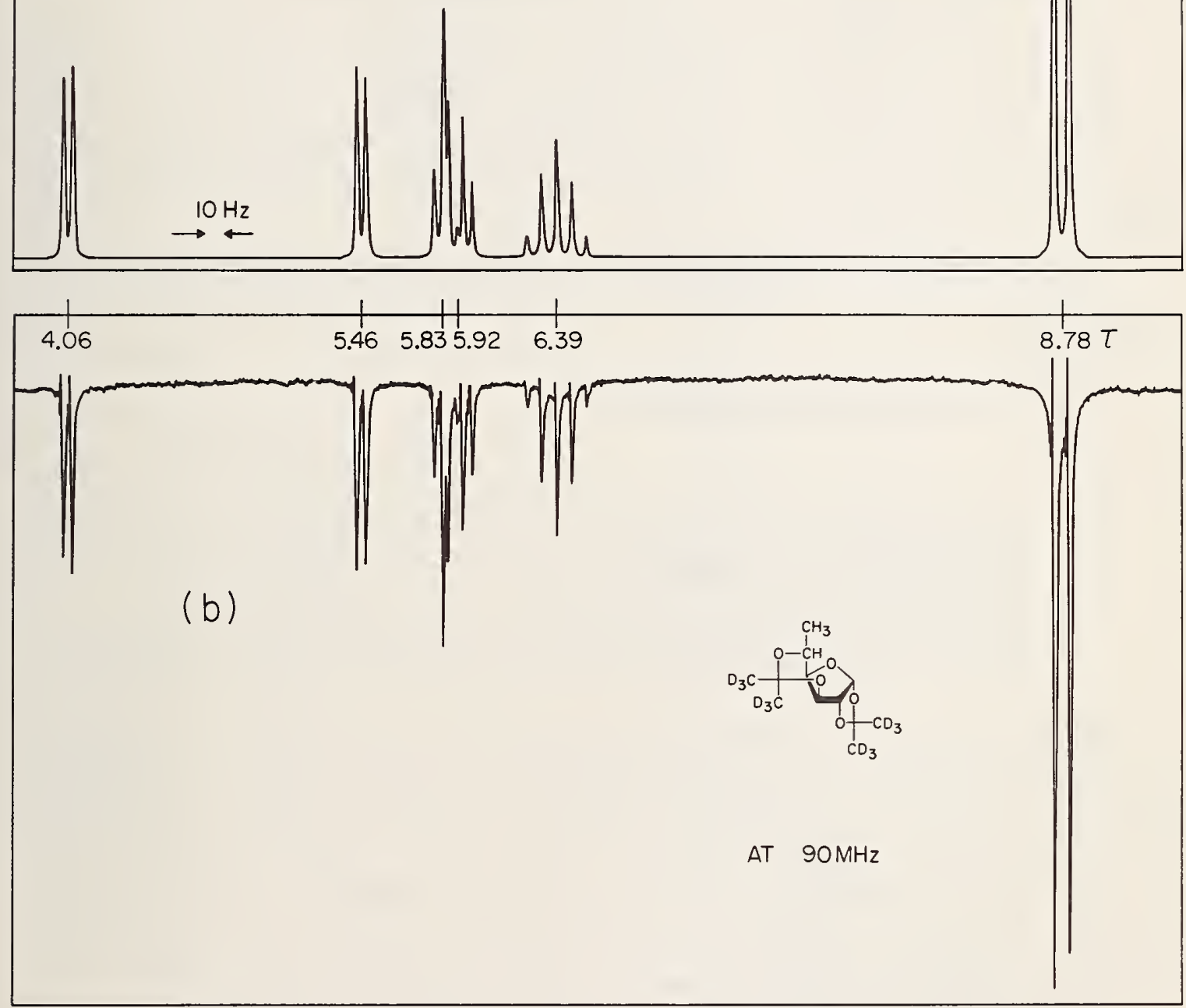

Figure 9. P.m.r. spectra of 2. (a) Computed spectrum after iterative analysis ${ }^{2}$ y magnetic-equivalence

factoring, and ( $b$ ) observed spectrum of solution in $8: 1(\mathrm{v} / \mathrm{v})$ acetone-d 6 -benzene at $90 \mathrm{MHz}$. 
were $0.240,0.083,0.080$, and 0.080 , and the final probable errors of parameters (see tables 24 and 25 ) were $\leqslant 0.028$.

The values of $J, 5$ shown in table 24 are considerably larger than those of the 1,2:3,5-di-0-arylidene and -alkylidene derivatives studied previously $[48,63,64]$; this indicates that the chair shapes of the m-dioxane rings of $\underset{\sim}{1}, \underset{\sim}{1}-{ }^{15} N$, and $\underset{\sim}{2-d_{12}}$ have either been modified in some way, or are involved in conformational equilibria different from those of the derivatives previously studied. This difference may be attributable to the presence of 1:3-syn-diaxial $\mathrm{CH}_{3}-\mathrm{CH}_{2} \mathrm{~N}$ or $\mathrm{CD}_{3}-\mathrm{CH}_{3}$ interactions in the anticipated chair forms of $\underset{\sim}{1}, \underset{\sim}{1}-15 \mathrm{~N}$, and $\underset{\sim}{2}-d_{12}$.

Further work is planned, using variable-temperature techniques.

F. Cyclic Polyhydroxy Compounds

(A. J. Fatiadi)

1. Determination of Inososes with an Alkaline Solution of Copper(II) Oxalate-Tartrate Complex (the Somogyi Reagent); Structure of the Reagent (by E.s.r. Studies), and Reaction Mechanisms Involved

Four pentahydroxycyclohexanones (inososes) have been analyzed with the Somogyi reagent, and empirical equations for their quantitative determination have been derived. It was found that reaction of the Somogyi reagent with inososes affords nonstoichiometric quantities of cuprous oxide, and that the reducing power varies with the inosose.

The following inososes were analyzed and equations for each were determined (see table 26): a racemic mixture of $2 \underline{\underline{D}}-2,3,4,6 / 5$-pentahydroxycyclohexanone (D-epi-inosose-2) ( $\underset{\sim}{\sim}$ ) and $2 \mathrm{~L}-2,4,5,6 / 3$-pentahydroxycyclohexanone (L-epiinosose-2) (2) , the L enantiomorph $\underset{\sim}{2}, 2,4,6 / 3,5-$ pentahydroxycyclohexanone (myo-inosose-2) (

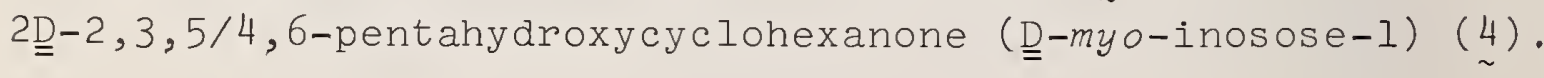


\&े

(1) $\stackrel{0}{>} \approx$

or 0 工

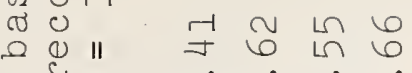

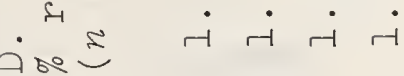

نे

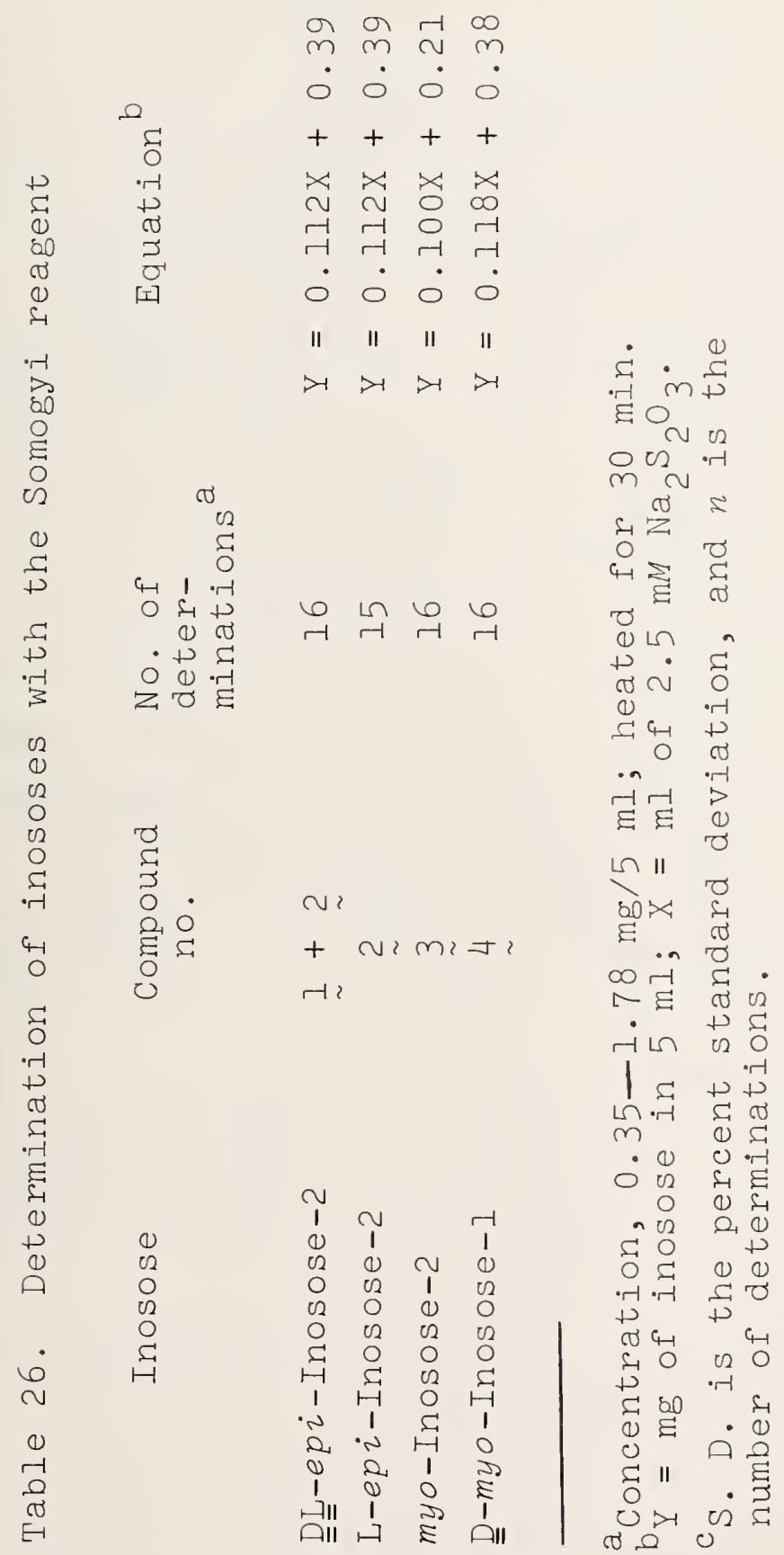




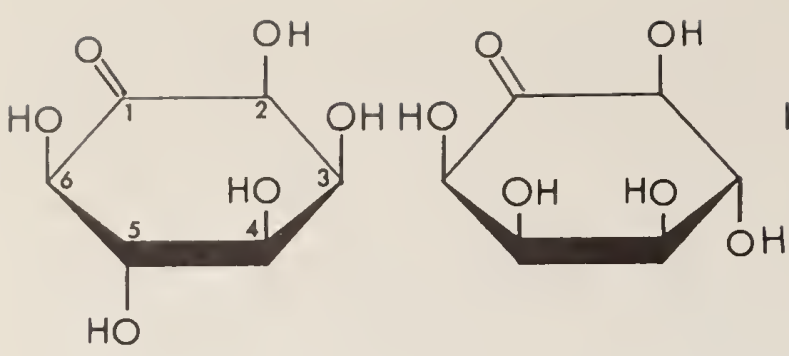

1
2

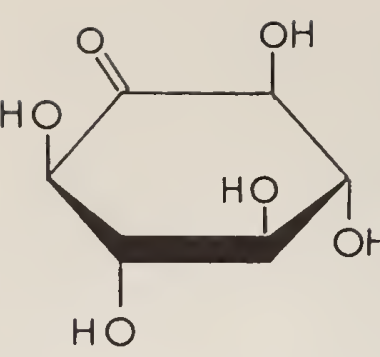

3
4

An e.s.r. study of the Somogyi reagent (from aqueous solution at $77 \mathrm{~K}$ ), described later, showed that the $\mathrm{Cu}(\mathrm{II})$ complex present in the reagent has mainly a monomeric structure. By comparison, the e.s.r. spectrum of $\mathrm{Cu}$ (II) complexes at $77 \mathrm{~K}$ of the deep-blue Fehling reagent revealed a monomer-dimer structure, that of the Benedict reagent showed the presence of a relatively low concentration of dimer as compared to monomer, and that of the Reeves cuprammonium reagent showed primarily the monomeric structure. Spectrophotometric and e.s.r. studies of the mechanisms of oxidation of inososes with the Somogyi reagent at moderate temperature are in agreement with a one-electron transfer process; however, at higher temperature, an extensive degradation of the inosose by the Somogyi reagent is doubtless caused by generation of transient radicals during the oxidation, as evidenced by results of a radical-trapping experiment

The mechanisms suggested for the oxidation of inososes with the Somogyi reagent at high temperature (involving a transient radical intermediate) and at moderate temperature (a one-electron, transfer process) are shown in schemes 1 and 2 , respectively.

These results have been incorporated in an article that has been submitted for publication. 
<smiles>[R]C1C(=O)C(O)C([R])C([R])C1[R]</smiles>

$\stackrel{\mathrm{OH}^{-}}{\rightleftharpoons}$

2

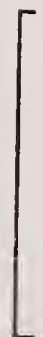<smiles>[R]C1=C([O-])C([O-])=C([R])C([R])C1[R]</smiles>

$\frac{\mathrm{Cu}^{2+}}{\mathrm{O}_{2}}$

5

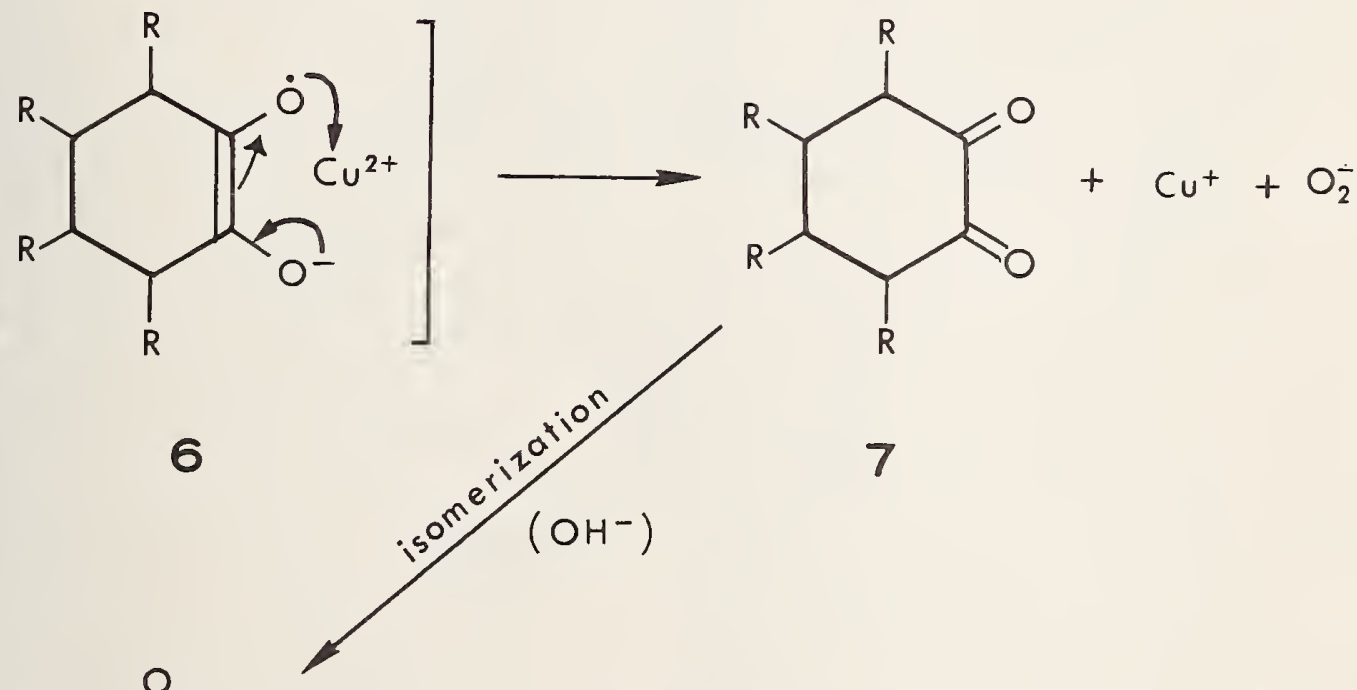<smiles>[R]C1C(=O)C(O)=C(O)C([R])C1[R]</smiles>

$\longrightarrow \mathrm{OH}^{-}$

$R=H, O H$

$\mathbf{R}^{\prime}=\mathrm{OH}$<smiles>[R]c1cc([R])c([R])c([R])c1[R]</smiles>

9

Scheme 1 


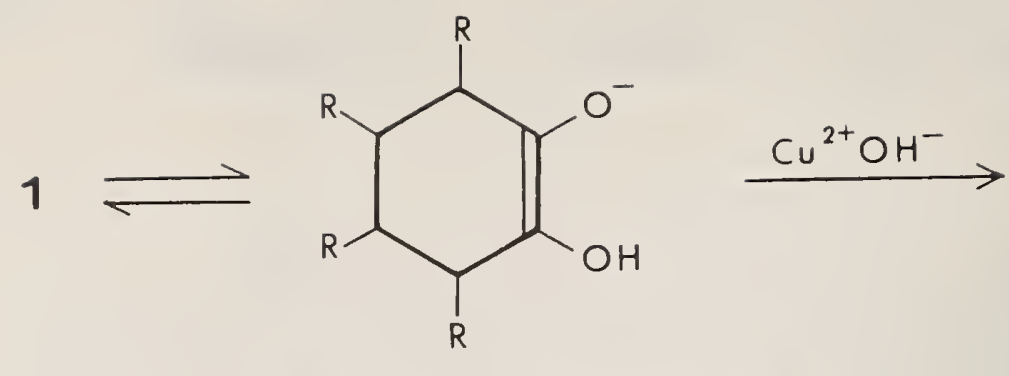

10

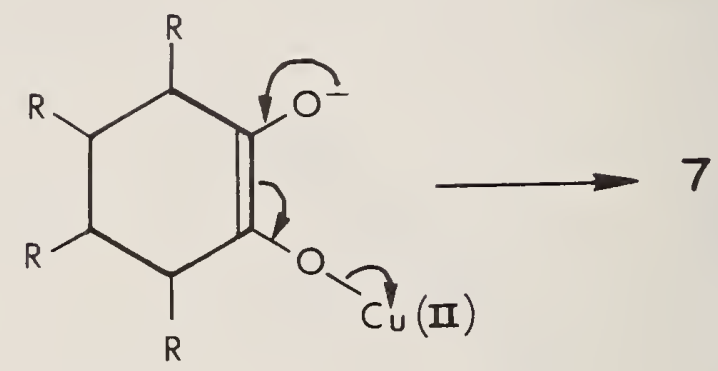

11

$$
R=H, O H
$$

Scheme 2 
2. E.s.r. Evidence for a Dimer Structure of the Alkaline

Copper(II) Tartrate Complex in the Fehling Reagent at $77 \mathrm{~K}$

Application of low-field e.s.r. spectroscopy to structural study of certain frozen Cu(II) complexes [69] and vanadyl complexes [70] prompted our work. The e.s.r. spectra of polycrystalline, frozen $(77 \mathrm{~K})$ aqueous solutions of four reagents commonly used in the carbohydrate field are shown in fig. 10. The low-field lines of the e.s.r. spectrum of frozen Fehling reagent (spectrum B) are evidence of some partially resolved structure; this low-field portion (1.0-2.0 kgauss) of the spectrum can be attributed to forbidden transitions associated with $\Delta \mathbb{M}=2$ components (dimer structure) $[70,71]$, and can arise from $\mathrm{Cu}^{2+}-\mathrm{Cu}^{2+}$ ion-pairs [69]. The $\Delta \mathrm{M}=2$ transitions occurring as the result of $\mathrm{Cu}^{2+}-\mathrm{Cu}^{2+}$ dipole interaction should appear at approximately half the field (about 1.6 kgauss, $g$ 4 ) of the $g$ 2 ( 3.3 kgauss) [69-71], and the actual spectrum obtained at high gain (for spectrum B) shows a structure at about 1.5 kgauss which is characteristic of a dimer.

A possible structure for the dimer that exists in Fehling solution at $77 \mathrm{~K}$ is one in which two tartrate hydroxylcarboxylate ligands $\left(\mathrm{C}_{4} \mathrm{H}_{2} \mathrm{O}_{6}\right)$ briage the $\mathrm{Cu}^{2+}(\mathrm{OH})-\mathrm{Cu}^{2+}(\mathrm{OH})$ ion-pair to give two five-membered, chelate rings; a polynuclear, complex structure for this reagent had been suggested without proof [72].

The e.s.r. spectrum of the Benedict reagent at $77 \mathrm{~K}$ (spectrum D) shows a broad, rather weak, low-field signal that is somewhat shifted to higher field ( 1.5 to 2.0 kgauss); its presence can be ascribed to a relatively low concentration of dimer, as compared to monomer, although the dimer structure 


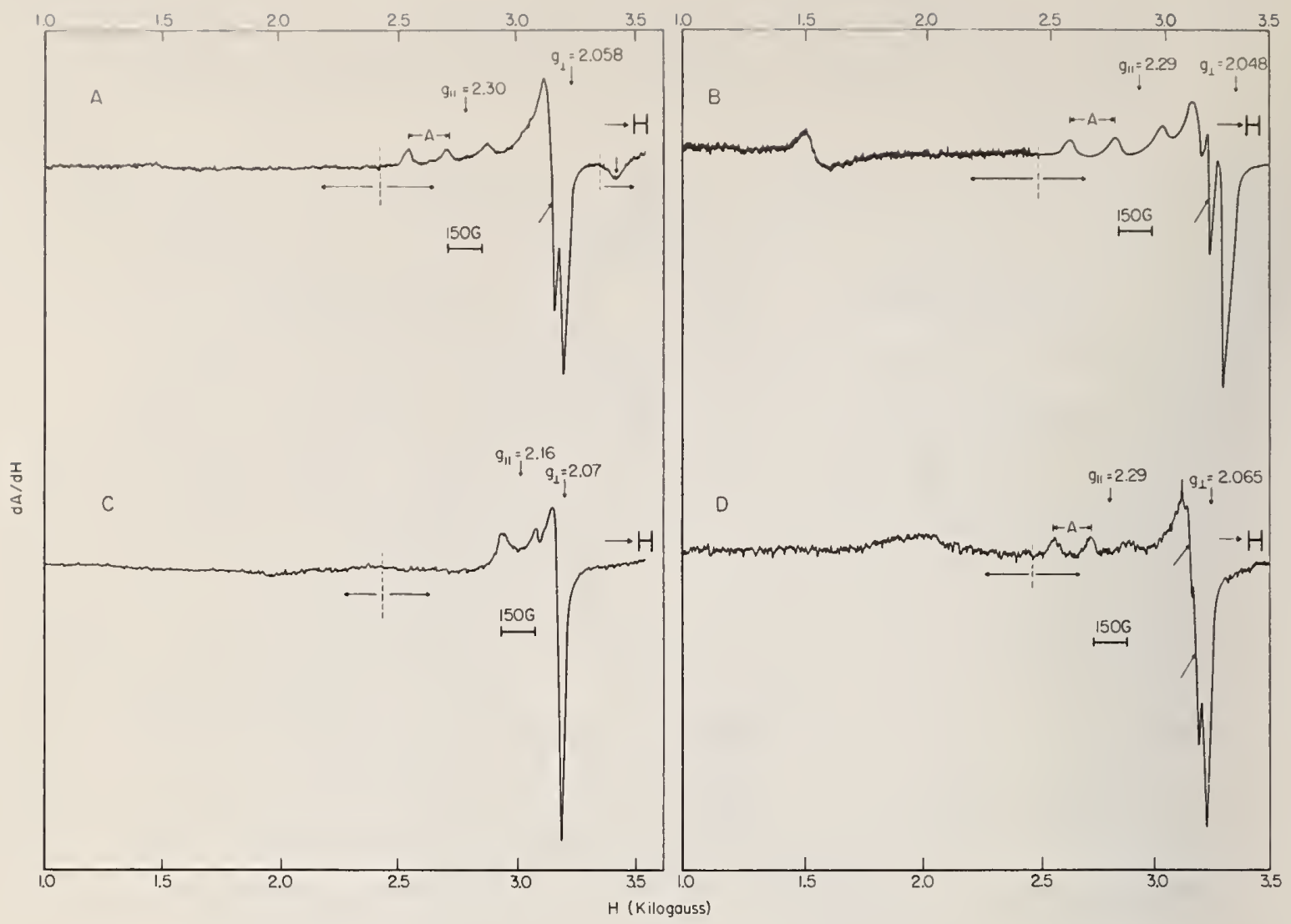

Figure 10. E.s.r. spectra of frozen $(77 \mathrm{~K})$ aqueous alkaline solutions of $\mathrm{Cu}$ (II) complexes: spectrum $A$, tartrate-oxalate (the Somogyi reagent); B, tartrate (the Fehling reagent); C, citrate (the Benedict reagent); and $D$, tetraamine hydrate (cuprammonium, the Reeves reagent). All four low-field e.s.r. spectra on the left side of the dotted line were recorded at somewhat greater field-modulation amplitude than the spectra for the $g \sim 2$ region. 
was found [73] for $\mathrm{Cu}(\mathrm{II})$ citrate complex at different $\mathrm{pH}$ values. The very weak (or absent) low-field lines in the e.s.r. spectra of the Somogyi (spectrum A) and Reeves reagent (spectrum $\mathrm{C}$ ) at $77 \mathrm{~K}$ are indicative of a preponderantly monomeric structure for these two $\mathrm{Cu}(\mathrm{II})$ complexes.

The spectrum of the monomeric species $(\Delta \mathrm{M}=1)$ shows a characteristic, single, intense, perpendicular component, and less-intense, four-line, parallel components in the $\mathrm{g}$ u2 region. Some overlap is observed in all four e.s.r. spectra, but it is particularly severe in spectrum C. The magnetic parameters for the monomeric species are given in table 27 .

Table 27. Magnetic parameters ${ }^{a}$ for the $\mathrm{Cu}(\mathrm{II})$ complexes of the Somogyi (A), Fehling (B), Reeves (C), and Benedict (D) reagents at $77 \mathrm{~K}$
Reagent
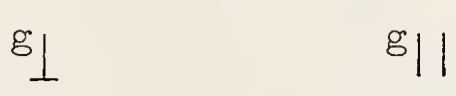
$\mathrm{A} \|, \mathrm{cm}^{-1} \times 10^{-4}$
A
$2.058 \pm 0.003$
$2.302 \pm 0.005$
$164 \pm 10$
$\mathrm{B}$
$2.048 \pm 0.004$
$2.294 \pm 0.003$
$188 \pm 10$
C
$2.072 \pm 0.001$
D
$2.065 \pm 0.003$
$2.291 \pm 0.002$
$165 \pm 5$

andicated limits of error for the $g$ and $A$ values are maximum estimated errors.

The presence of extra features in the high-field portion $(\Delta M=1)$ of the e.s.r. spectra for $A, B$, and $D$ (see fig. 10, indicated by arrows) could readily be confused with the perpendicular components from ${ }^{6} \mathrm{Cu}$ and ${ }^{65} \mathrm{Cu}$ nuclei; however, it is known $[74,75]$ that separation of lines due to ${ }^{6}{ }^{3} \mathrm{Cu}$ and ${ }^{65} \mathrm{Cu}$ is a difficult task that requires a special technique. However, the extra peak in the e.s.r. spectrum of the Fehling reagent $(77 \mathrm{~K})$ was also observed for two samples of the reagent prepared from salts of pure ${ }^{6}{ }^{3} \mathrm{Cu}$ and ${ }^{6}{ }^{5} \mathrm{Cu}$ isotopes, respectively. 
When, for either the Somogyi or the Fehling reagent, the frozen solution is warmed to room temperature, an extra signal is produced (at $3.245 \mathrm{kgauss}$ for $\mathrm{A}$ ), in addition to the usual, four-line, e.s.r. spectrum of the cu(II) complex. The origin of this and other extra signals may be associated with the presence of paramagnetic superoxide, ion-ligand $\mathrm{O}_{2}^{-}$.

The author thanks T. Murphy for specimens of salts of pure ${ }^{6}{ }^{3} \mathrm{Cu}$ and ${ }^{65} \mathrm{Cu}$, and $\mathrm{N}$. Adams for the e.s.r. measurements.

\section{Preparation of Inositol Hexasulfate}

Inositol hexasulfate $(\underset{\sim}{I})$ is conveniently prepared by direct interaction of myo-inositol either with fuming sulfuric or chlorosulfonic acid. Hexasulfate $\underset{\sim}{l}$ was isolated

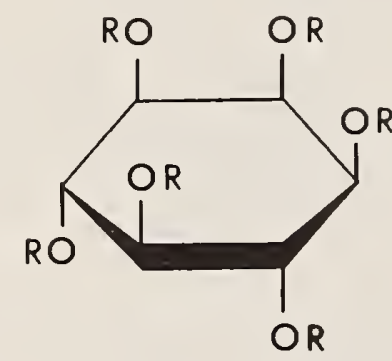

$$
\begin{array}{ll}
1 & \mathrm{R}=\mathrm{SO}_{3} \mathrm{H} \\
2 & \mathrm{R}=\mathrm{SO}_{3} \mathrm{~K}
\end{array}
$$

from the reaction mixture as its hexapotassium salt (2) in 55--62\% yield by (a) an exchange reaction with potassium chromate, or (b) direct neutralization with potassium hydroxide.

Fuming sulfuric acid containing $30 \%$ of sulfur trioxide was placed in an Erlenmeyer flask provided with a magnetic stirring-kar, a thermometer extending to the bottom of the flask, and a loosely stoppered drying-tube containing calcium chloride. The acid was heated to $60{ }^{\circ} \mathrm{C}$ (oil bath), 
and finely powdered myo-inositol was introduced in small increments during $2.75-3 \mathrm{~h}$. The reaction is exothermic, and the temperature of the reaction mixture should be kept at $60-65^{\circ} \mathrm{C}$ by cooling. If the temperature exceeds $80^{\circ} \mathrm{C}$, some decomposition usually ensues, as shown by darkening of the solution. Sometimes, near the end of the reaction, the temperature must be raised to $70-75^{\circ} \mathrm{C}$ for 10 to $15 \mathrm{~min}$, in order to dissolve any unreacted material. The light-yellow to brownish yellow solution containing myo-inositol hexasulfate was then cooled to about $35^{\circ} \mathrm{C}$, and was carefully poured into a stirred slurry of a solution of potassium chromate (10\% excess) in water with crushed ice. The greenish yellow suspension was then diluted with cold methanol, stirred for $30 \mathrm{~min}$ at $0-3^{\circ} \mathrm{C}$, and filtered. The precipitate was washed successively with ice-cold water and ice-cold l:l water-methanol. The wet, somewhat colored product was then recrystallized from hot water; cooling and washing produced snow-white needles of 2 (as the dihydrate when dried in vacuo at room temperature); yield 53--55\%; the test for $\mathrm{SO}_{4}^{2-}$ by addition of $\mathrm{Ba}^{2+}$ was negative. This procedure has been published [76].

3. GENERAL ORGANIC CHEMI STRY

(A. J. Fatiadi)

A. Novel Iodination of the a-Methylene Group in 1,3Diphenyl-1,3-propanedione and Related $\beta$-Diketones with Periodic Acid

In continuation of a study [77] of new applications of periodic acid in organic chemistry [78], uses of this oxidant have now been extended to the specific iodination of the $\alpha$-methylene group of certain $\beta$-diketones. 
A solution of 1,3-diphenyl-1,3-propanedione ( $\underset{\sim}{1} \underset{\sim}{ }$, enol content in chloroform-d, $292 \%$, by p.m.r. spectroscopy) in acetic acid was stirred with an aqueous solution of paraperiodic acid at $95^{\circ} \mathrm{C}$ for $15 \mathrm{~min}$, and then at room temperature for $60 \mathrm{~min}$. The mixture was next treated with water at room temperature to yield snow-white needles of the 2-iodo derivative $\underset{\sim}{1 b}$.<smiles>[R]C([R])(C(=O)c1ccccc1)C(=O)c1ccccc1</smiles>

1

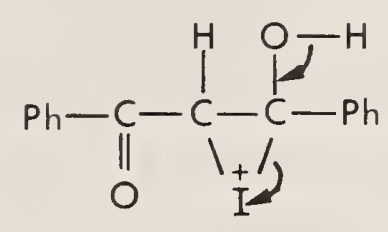

2

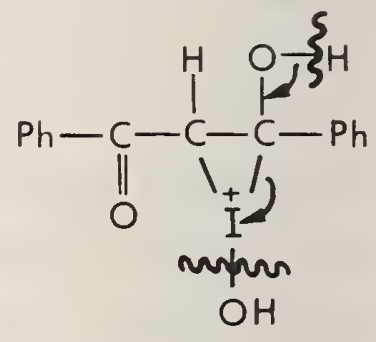

3
a, $R=R^{\prime}=H$
b, $R=H ; R^{\prime}=I$
c, $R=R^{\prime}=I$
d, $R=R^{\prime}=B r$

The mass spectrum (probe-temperature, $30^{\circ} \mathrm{C}$ ) of $\underset{\sim}{\operatorname{l}} \underset{\sim}{\mathrm{b}} \mathrm{did}$ not show the molecular ion $(\mathrm{m} / \mathrm{e}$ 350), but did exhibit an ion peak at $m / e 223$ due to elimination from $\underset{\sim}{\operatorname{lb}}$ of iodine, observed as a molecular ion at $m / e 254\left(I_{2}{ }^{2+} \tilde{r}^{\sim}\right.$ and as an ion peak at $\mathrm{m} / \mathrm{e} 127\left(\mathrm{I}^{+}+\mathrm{HI}\right)$. The molecular ion $(\mathrm{m} / \mathrm{e} 350)$ was observed at higher probe-temperature $\left(40^{\circ} \mathrm{C}\right)$. Compound $\underset{\sim}{\mathrm{lb}}$ showed $\lambda_{\max }^{\mathrm{MeOH}} 250\left(\varepsilon_{\mathrm{mM}} 18.5\right)$ and $330 \mathrm{~nm}\left(\varepsilon_{\mathrm{mM}} 1\right) ; \lambda_{\max }^{\mathrm{KBr}} 5.90$ and 6.00 ( $C=0$, non-hydrogen-bonded), 15.30 and $16.68 \mu \mathrm{m}$ $\left(-{ }_{1}^{\prime}-I\right)$; p.m.r. data in $\mathrm{CDCl}_{3}, \delta 7.30-8.20$ (m $10 \mathrm{H}$, aromatic) and $\delta 6.97$ ( $\mathrm{s}, 1 \mathrm{H},-\mathrm{CH}=\mathrm{C}^{\prime}$ ). The monoiodo derivative $\underset{\sim}{\mathrm{l}} \mathrm{b}$ was also obtained (in $50 \%$ yield) on treatment of $\underset{\sim}{1} \underset{\sim}{\text { in acetic }}$ acid with an aqueous solution of iodic acid at $95{ }^{\circ} \mathrm{C}$ for $20 \mathrm{~min}$ and at room temperature for $24 \mathrm{~h}$. 
Treatment of an acetic acid solution of $\underset{\sim}{\operatorname{la}}$ with an aqueous solution of paraperiodic acid at $95^{\circ} \mathrm{C}$ for $30 \mathrm{~min}$ and at room temperature for 30 min gave yellow crystals of the 2,2-diiodo derivative $(\underset{\sim}{\operatorname{lc}} \underset{\sim}{\mathrm{c}})(19--21 \%), \mathrm{m} \cdot \mathrm{p} \cdot 152--153{ }^{\circ} \mathrm{C}(95 \%$ EtOH). Compound $\underset{\sim}{l c}$ exhibits moderate stability (loss of iodine occurs, to give $\underset{\sim}{l b}$ ) on storage as the solid, or in solution; it shows moderate loss of iodine on recrystallization from ethanol. The mass spectrum (LKB-9000, probe-temperature, $60{ }^{\circ} \mathrm{C}$ ) exhibited a molecular-ion peak at m/e 476; other spectral data: $\lambda_{\max _{\mathrm{KR}}}^{\mathrm{MeOH}} 248\left(\varepsilon_{\mathrm{mM}} 3.1\right), 290(\mathrm{sh})$ $\left(\varepsilon_{m M} 4.9\right)$, and $440 \mathrm{~nm}\left(\varepsilon_{\mathrm{mM}} 0.2\right) ; \lambda_{\max } 6.03(\mathrm{C}=0$, nonhydrogen-bonded), $15.28,16.28$, and $17.65 \mu \mathrm{m}(-\mathrm{C}-I) ; \mathrm{p} . \mathrm{m} . \mathrm{r}$. data in $\mathrm{CDCl}_{3}, 87.20-8.10(\mathrm{~m}, 10 \mathrm{H}$, aromatic).

When iodic acid was used instead of periodic acid, little or no formation of the diiodo derivative $\underset{\sim}{\operatorname{lc}}$ was observed. The use of a larger proportion of the reagents (iodic or periodic acid) did not improve the yields of the products $\underset{\sim}{1 b}$ or $\underset{\sim}{l c}$.

Compounds $\underset{\sim}{1 \mathrm{~b}}$ and $\underset{\sim}{1 c}$ were readily converted into the known dibromo derivative $\underset{\sim}{l} \underset{\sim}{d}, \mathrm{~m}$ p $94--96{ }^{\circ} \mathrm{C}$, on treatment of their solution in carbon tetrachloride with bromine at room temperature. Compounds $\underset{\sim}{\operatorname{lb}}$ and $\underset{\sim}{\operatorname{lc}}$ were converted into the starting material $\underset{\sim}{l a}$ on extraction of their chloroform solutions with an aqeuous solution (5\%) of sodium hydrogen sulfite.

On treatment of solutions of the foregoing compounds in methanol with a solution of cupric acetate in methanol, the bis-copper chelate complex was precipitated, immediately for $\underset{\sim}{\mathrm{la}}$, after three min for $\underset{\sim}{\mathrm{lb}}$, and not at all after $24 \mathrm{~h}$ (a deep-green solution) for compounds $\underset{\sim}{l} \underset{\sim}{c}$ and $\underset{\sim}{l} \underset{\sim}{d}$; this test serves to distinguish between these compounds. The progress of formation of the product was monitored by p.m.r. spectroscopy $\left(\mathrm{CDCl}_{3}\right)$ as follows: for disappearance of the enolic

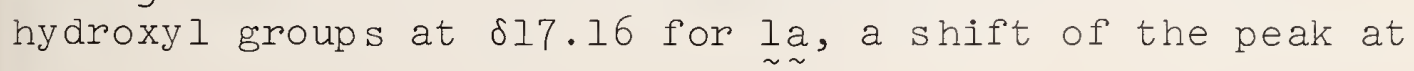


$\delta 6.82\left(-\mathrm{CH}=\mathrm{C}^{\prime}\right.$ ) for $\underset{\sim \sim}{\mathrm{la}}$ to $\delta 6.97\left(-\mathrm{CH}=\mathrm{C}_{-}^{-}\right)$for $\underset{\sim}{\mathrm{lb}}$, and disappearance of the latter for $\underset{\sim}{l} \mathrm{c}$.

Formation of the mono-iodo derivative from other acyclic (enolic) B-diketones has been observed; for example, from 1,3-diphenyl-1,3-butanedione, 2,4-pentanedione (which gives a vigorous reaction), 2,4-hexanedione, hexafluoro-2,4pentanedione, and others.

Application of $N$-iodosuccinimide, used in iodination of the methine group of certain enol acetates [79], or of other iodinating reagents [80], failed to iodinate the methylene group in $\underset{\sim}{1} \mathrm{a}$. When sodium metaperiodate was used instead of periodic acid, or the acetic acid was replaced by $N, N$-dimethylformamide or $p$-dioxane, none of the reactions mentioned were observed.

E.s.r. monitoring of the reaction of periodic acid with $\underset{\sim}{l_{\sim}}$ showed no radical participation, indicating the ionic character of the iodination process. The behavior of periodic (or iodic) acid towards $\underset{\sim}{\text { la indicates structural }}$ lability of the I--O bond in the reagents. This high lability of the I-O bond may be associated with a relatively weak, double-bond character in the $I-0$ bond [81]. Iodination of $\underset{\sim \sim}{\mathrm{la}}$ can be envisaged as involving addition of the iodonium ion to the double bond, to give the intermediate 2 , although addition of a molecule of hypoiodous acid, or its anion (an iodoso ion $\mathrm{IO}^{-}$), with subsequent elimination of a molecule of water to give $\underset{\sim}{l_{\sim}}$, as shown in intermediate $\underset{\sim}{3}$, is not excluded. The relatively modest yield of the iodo compounds may be due to an association of the enolic diketone with the reagent [82] that could impede formation of intermediates $\underset{\sim}{2}$ and $\underset{\sim}{3}$. The results were incorporated in an article that has been published [83]. 
B. Degradation of Bilirubin with Chromic Acid

Bilirubin $(\underset{\sim}{1})$ was suspended in acetone, cooled to about

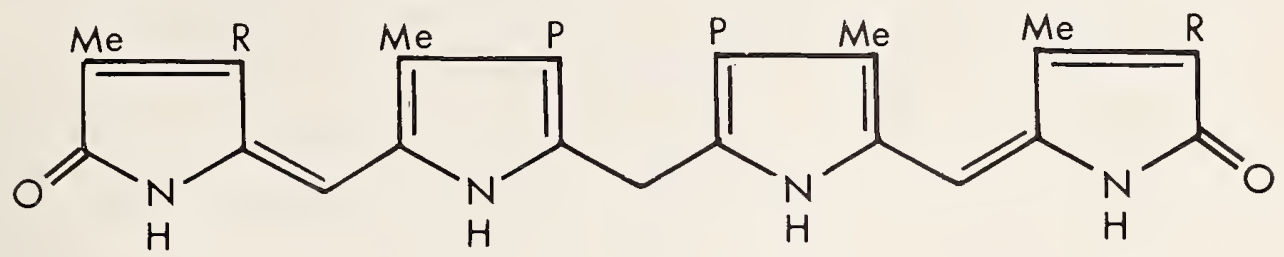

Bilirubin

$$
\text { 1. } \begin{aligned}
R & =-\mathrm{CH}=\mathrm{CH}_{2} \\
P & =-\mathrm{CH}_{2} \mathrm{CH}_{2} \mathrm{CO}_{2} \mathrm{H}
\end{aligned}
$$

$10{ }^{\circ} \mathrm{C}$, and treated with chromic acid [84-86] $\left(1 \% \mathrm{CrO}_{3}\right.$ in $2 \mathrm{M}$ sulfuric acid). The suspension was stirred for two hours; by this time, all solids had dissolved to give a deep-brown solution. The latter was extracted with ether, dried (sodium sulfate), concentrated to a thin syrup, neutralized with aqueous sodium carbonate, and filtered. The clear filtrate was extracted with 4:I (v/v) ether-dichloromethane, and the extract was washed with water, dried (sodium sulfate), and set aside (solution A) for isolation of $\underset{\sim}{3}$. The alkaline extract was neutralized ( $6 \mathrm{M}$ hydrochloric acid) to about $\mathrm{pH} 2$ and extracted with ether. The extract was washed, dried, evaporated to a syrup, and left to crystallize in an open dish at room temperature for $72 \mathrm{~h}$, to give crystalline, crude hematinic acid $\underset{\sim}{(2)}$, m p 96-98 ${ }^{\circ} \mathrm{C}$. The product was dissolved in ether, the suspension was filtered (carbon), and the filtrate chromatographed on a column ( $1 \times 20 \mathrm{~cm}$ ) of silica gel, and eluted with $1: 5$ ( $/ \mathrm{v}$ ) ether-pentane; a middle fraction was collected, evaporated to dryness, and the crystals recrystallized from etherpentane (carbon); white crystals of 2 , $m$ p $110--112{ }^{\circ} \mathrm{C}$; lit. [87] $\mathrm{m} \mathrm{p} 112--113^{\circ} \mathrm{C}$. 


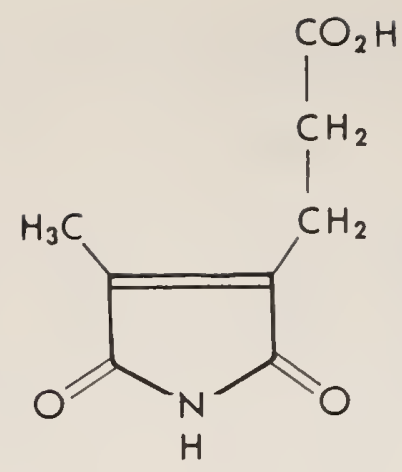

Hematinic acid

2

Extract A was evaporated to dryness, the product dissolved in ethyl acetate, and the solution chromatographed on a column ( $1 \times 20 \mathrm{~cm}$ ) of Florisil by elution with $1: 6(\mathrm{v} / \mathrm{v})$ ethyl acetate--pentane and then with $1: 3$ (v/v) ethyl acetatepentane. A syrupy product was obtained; on crystallization from $\sim 60 \%$ aqueous ethanol (carbon) and cooling (72 h), it gave microcrystalline needles of ${ }_{\sim}$, m p $66--67{ }^{\circ} \mathrm{C}$; lit [87] $\mathrm{m}$ p $68-69^{\circ} \mathrm{C}$. Compound $\underset{\sim}{3}$ was homogeneous by t.I.c. [Silica

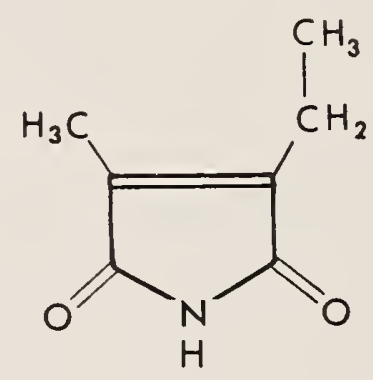

2-Ethyl-3-methylmaleimide

3 
Gel G; l:5:3 (v/v) cyclohexane-carbon tetrachloride-ethyl acetate (solvent A); with a spray of $3 \%$ solution of benzidine in methanol]; $R_{F} 0.44 \pm 0.02$, close to that reported $[85,86]$.

It is interesting that the oxidation mixture from $\underset{\sim}{I}$ (containing five or six components by t.l.c.) included a substantial proportion of an unknown component showing a strong blue fluorescence; the presence of a component with this property was not reported by Rüdiger and co-workers [84-86] in their comprehensive study of degradation products of bilirubin and biliverdine derivatives. The presence of this component was also observed in degradation products of bilirubin and hemin with periodic acid (see later).

The unknown fluorescent component has the following $R_{F}$ values: solvent A [85], $0.76 \pm 0.03$, solvent B $[4: 1(\mathrm{v} / \mathrm{v})$ benzene-ethyl acetate], $0.70 \pm 0.02$. The unknown compound can be isolated by use of a Florisil column with solvent $B$.

C. Degradation of Hemin and Related Bile Pigments with

Periodic Acid

Bile pigments are usually degraded by chromic acid $[85,86]$; however, it has been found in this laboratory that aqueous solutions of periodic acid and even iodic acid can oxidize porphyrins (hemin, hematin, heme), as well as bile pigments (bilirubin, biliverdine, urobilin, and others). Usually, the co-solvent used in these oxidations is acetic acid, tetrahydrofuran, or acetone. Although acetone in the presence of periodic acid and oxidizable substrate produces substituted iodo-acetones, degradation of hemin $(\underset{\sim}{4})$ in the presence of this solvent proceeds faster than in the other solvents. However, for a systematic study of the oxidation of bile pigments with periodic acid, use of tetrahydrofuran-acetic acid as co-solvent was found advantageous. The degradation products are usually identified by t.l.c. (activated Silica 


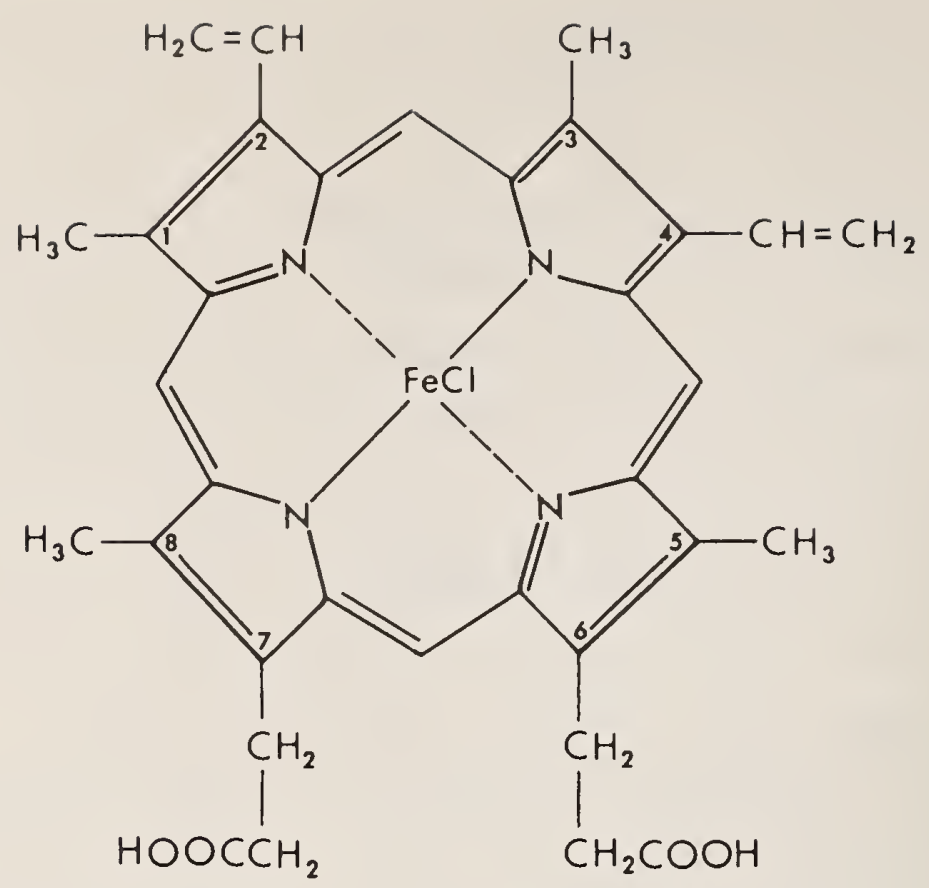

Hemin (chlorohemin)

4

Gel G, $20 \times 20 \mathrm{~cm}$ or $5 \times 20 \mathrm{~cm}$ plates, solvents $A[85,86]$ or $B$, and $3 \%$ benzidine solution in methanol as the spray $[85,86]$ to give fluorescent spots), by comparison of their $R_{F}$ values with those of authentic samples and with those reported in the literature.

Hemin was suspended in acetone, treated with $4 M$ aqueous periodic acid and stirred for $3 \mathrm{~h}$ at room temperature. The white solid precipitated was filtered off, and washed with acetone, and the filtrate was neutralized with $10 \%$ aqueous sodium hydrogen carbonate. The neutral solution was extracted with 4:1 (v/v) ether-dichloromethane, and the extract was thoroughly washed with water, dried (sodium sulfate), and evaporated to give a very pungent syrup (neutral fraction). 
T.I.c. analysis of the neutral fraction showed components having the following $R_{F}$ values (solvent $A[85,86]$ ): (a) before spraying with benzidine spray, $R_{F} 0.74$ (blue fluorescence) and 0.41 (strong blue fluorescence); (b) after spraying with the benzidine spray, $R_{F} 0.91$ (yellow with purple fluorescence), 0.74 (blue fluorescence), 0.44 (yellow), 0.41 (blue fluorescence), 0.37 (red-purple), 0.30 (weak yellow), 0.24 (strong purple-brown), 0.17 (weak yellow), 0.075 (strong purple), and 0.00 (at origin). The spot having $R_{F} 0.44$ corresponds to compound $\underset{\sim}{3}[85,86]$, and that of $R_{F}$ 0.24 , to 2-ethylidene-3-methylsuccinimide $[85,86]$.

On acidification of the sodium hydrogen carbonate solution with hydrochloric acid, extraction with ether, and evaporation, a small amount of crude 2 was isolated.

D. Isolation of a Biliverdine Type of Reaction Intermediate on Oxidation of Bilirubin with Periodic Acid

Treatment of a solution of bilirubin ( $\underset{\sim}{(})$ in methyl sulfoxide with an aqueous solution of periodic acid, with stirring at $15--20{ }^{\circ} \mathrm{C}$ for $10 \mathrm{~min}$, gives at first a dark-green solid which can be filtered off (prolonged treatment of this solid with periodic acid causes degradation). The solid can be recrystallized from methanol-ether, to give a bluish green, microcrystalline solid $\underset{\sim}{5}\left(\lambda_{\max }^{\mathrm{MeOH}} 226,272 \mathrm{sh}\right.$, 340,372 , and $655 \mathrm{~nm}$ ) having a biliverdine type of absorption; the product contains a free iodine atom and is believed to be a charge-transfer complex.

A sample recrystallized from methanol-pentane (room temperature) and dried at $110{ }^{\circ} \mathrm{C} / 10 \mathrm{mtorr}$ for $2 \mathrm{~h}$ had $\mathrm{C}=53.3$, $\mathrm{H}=4.61, \mathrm{~N}=7.45$, and $I=16.1 \%$. However, the proportion of iodine $\left(I_{n}\right)$ in $\underset{\sim}{5}$ varies from one preparation to another, and this is characteristic of certain iodine charge-transfer compounds [88]. 


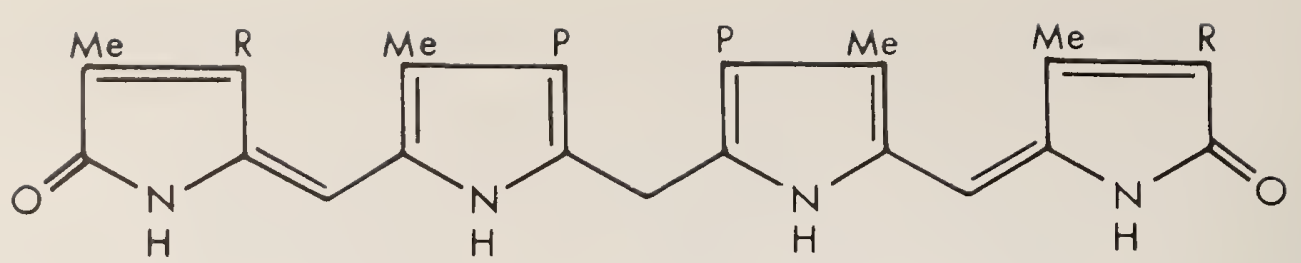

1, Bilirubin

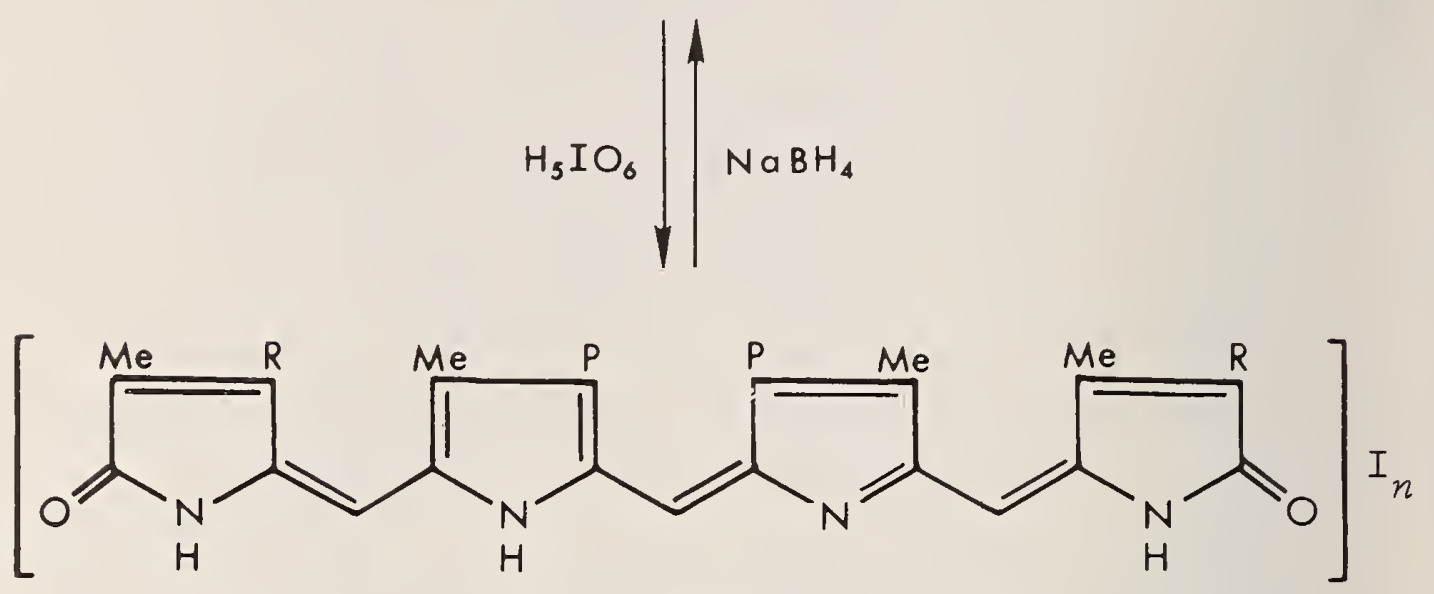

5, Billiverdine-iodine complex

$$
\text { where } \begin{aligned}
P & =-\mathrm{CH}_{2} \mathrm{CH}_{2} \mathrm{CO}_{2} \mathrm{H} \\
R & =-\mathrm{CH}=\mathrm{CH}_{2}
\end{aligned}
$$

Treatment of a methanol solution of complex $\underset{\sim}{5}$ with sodium borohydride in methanol at room temperature, followed by acidification with $3 M$ hydrochloric acid and extraction with chloroform, with thorough washing of the extract with water (which is imperative), gives bilirubin, $\lambda_{\max }^{\mathrm{CHCl}_{3}} 451 \mathrm{~nm}$ $\left(\varepsilon_{m M} 59.9\right)$, in $80 \%$ yield. This is the first report of a biliverdine derivative being reduced to bilirubin $(\underset{\sim}{I})$ without either the olefinic or carbonyl groups in $\underset{\sim}{l}$ being affected. A note on this study is in preparation for publication. 
4. DETECTION AND IDENTIFICATION OF TRACES OF ORGANIC COMPOUNDS

In NBS Technical Note 507, we reported some thin-layer chromatographic techniques for detecting low levels of two compounds, namely, 2,4,6-trinitrotoluene $(\underset{\sim}{I})$ and hexahydro1,3,5-trinitro-s-triazine (2). Alternative means for their

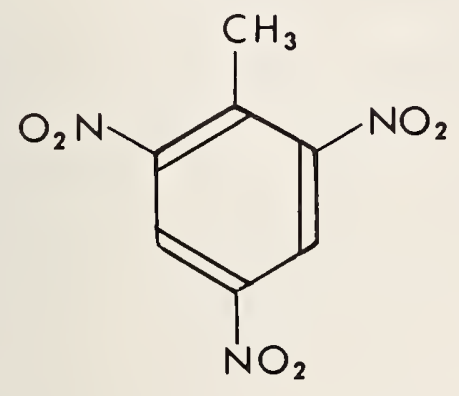

1

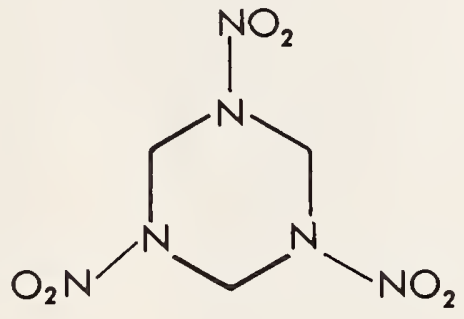

2

detection at low level have been investigated, where there seemed to be potential for lower levels of detection, or interferences with detection might be minimized or averted. A. $\quad 2,4,6$-Trinitrotoluene $(\underset{\sim}{1})$

\section{(A. Cohen)}

We had reported for compound $\underset{\sim}{I}$ that, after dévelopment on a silica gel plate with 9:l (v/v) benzene-pentane, and spraying of the dried plate with ethylenediamine in methyl sulfoxide [89], faint pink spots were detectable with as little as $15 \mathrm{ng}$ of compound. These spots did not fluoresce under 366-nm light. However, we now find that, when the sprayed plate is dipped in liquid nitrogen [90], and immediately examined under 366-nm light, the spots fluoresce (pink), and $10 \mathrm{ng}$ of $\underset{\sim}{1}$ is detectable. 
1. Detection of 2 by Diazonium Coupling

$$
\text { (A. Cohen) }
$$

With compound 2 , we had reported its detection at low levels by spraying the developed plate with methanolic potassium hydroxide and heating at $120{ }^{\circ} \mathrm{C}$ for $5 \mathrm{~min}$. This produces potassium nitrite, which can oxidize a sprayed-on solution of Benzidine Blue [91] to give weak-blue or purplish spots (nonfluorescent on cooling in liquid nitrogen). The production of $\mathrm{NO}_{2}^{-}$offers the potential for using a whole series of reagents, via the diazonium coupling-reaction, which could provide colored products of fluorescent compounds that might lead to still lower levels of detection. For example, after completing the treatment of $\underset{\sim}{2}$ to produce potassium nitrite, the plate can be sprayed with $0.5 \%$ of sulfanilic acid in $1: 1$ water-acetic acid and with $0.3 \%$ of $N, N$-dimethyl-l-naphthylamine in $1: I$ water--acetic acid [92]; in that publication, l-naphthylamine was used. Quantities comparable to those with the Benzidine Blue spray can be detected, but these spots are pink. They fluoresce when treated with liquid nitrogen and 366-nm light.

2. Detection of 2 by Formation of Formaldehyde (A. Cohen)

Formaldehyde is liberated from $\underset{\sim}{2}$ on treatment with concentrated sulfuric acid [93], and $J$ acid (6-amino-1naphthol-3-sulfonic acid) (0.5\%) in sulfuric acid [94] can detect the formaldehyde liberated. After spotting with 30 ng of compound 2 , developing as usual, spraying with the $J$ acid reagent, and warming for $8 \mathrm{~min}$ at $120{ }^{\circ} \mathrm{C}$, a very weak yellow fluorescence appears under 366-nm irradiation. This fluorescence is enhanced when the plate is immersed in liquid nitrogen and immediately examined under u.v. light. 


\section{Detection of 2 by Formation of Hydrazine}

$$
\text { (W. J. Rossiter, Jr.) }
$$

In conjunction with the preceding studies, additional experimentation on the detection of trace quantities of $\underset{\sim}{2}$ was attempted. It was proposed that hydrazine, generated by reduction of $\underset{\sim}{2}$ with zinc and acetic acid, might provide the basis for detection of $\underset{\sim}{2}$. There is literature precedence for the production of hydrazine from $\underset{\sim}{2}$ [95]: hydrazine had been isolated as aromatic azine derivatives from the reduction of 2 [95], as well as of hexahydro-1,3,5-trinitrosos-triazine [96] with zinc and aqueous base. In both cases, the reaction solutions had been neutralized with acid (and thus, possibly, acidified) before the addition of the aromatic aldehyde that trapped the hydrazine.

On exposure to acid vapors, aromatic nitro compounds are known to be reduced on t.l.c. plates coated with silica gel in which zinc has been incorporated [97]. This method was chosen for the reduction of $\underset{\sim}{2}$. Thus, after spotting with known quantities of $\underset{\sim}{2}$, zinc-containing t.l.c. plates were immersed in an atmosphere of acetic acid vapor for various lengths of time at various temperature; then the plates were removed and sprayed with an alcoholic solution of $p$-(diethylamino)benzaldehyde. Variations in the reaction conditions produced some plates on which, after spraying, orange spots appeared at points where 2 had originally been spotted. These orange spots were assumed to represent bis[p-(diethylamino)benzal]azine formed in the reaction of the generated hydrazine with $p$-(diethylamino)benzaldehyde. Maximization of the reaction conditions afforded detection of 2 at a level of $25 \mathrm{ng}$.

We wished to demonstrate rigorously that hydrazine was, indeed, being generated in the reduction and then visibilized as a colored, aromatic azine. This confirmation was accomplished by the combined techniques of t.l.c. and u.v.107 
visible spectrophotometry. By t.l.c., it was demonstrated that the colored spot generated on the plate from 2 migrated in different solvent systems with the same $R_{F}$ values as those displayed by authentic samples of the aromatic azines expected. Two azines, bis[p-(diethylamino)benzal]azine and salicylalazine, were used for this test. Next, when examined by u.v.-visible spectrophotometry, an extract of the orange spot generated on the t.l.c. plate from $\underset{\sim}{2}$, and assumed to be bis[p-(diethylamino)benzal]azine, exhibited the same, longwavelength, absorption band as an authentic sample of bis [p-(diethylamino)benzal]azine. This evidence, coupled with the literature data $[95,96]$ previously cited, leads to the conclusion that hydrazine is generated on the t.l.c. plate from reduction of 2 with zinc-acetic acid.

other known methods [98-104] for detection of hydrazine were next investigated, in an effort to improve the detection of $\underset{\sim}{2}$. Table 28 lists the various color-spot tests employed; as the table shows, only salicylaldehyde enhanced the detection of $\underset{\sim}{2}$; the lower limit of detection with this reagent is $10 \mathrm{ng}$ when the t.l.c. plate is examined under a 366-nm lamp immediately after immersion in liquid nitrogen. Without cooling in liquid nitrogen, the limit of detection with salicylaldehyde is $250-60 \mathrm{ng}$.

As $\underset{\sim}{2}$ was being detected at the lowest level by the fluorescence of salicylalazine, it was thought that extraction of the salicylalazine spot and fluorimetric analysis of the extract might possibly provide additional, and more conclusive, evidence for the presence of $\underset{\sim}{2}$. However, it was subsequently determined that salicylalazine does not fluoresce in solution [105]. Accordingly, a study was undertaken to find a substituted aromatic aldehyde or ketone that could be employed in the detection of trace quantities of 2 through the formation of an azine that does fluoresce in solution. of a number of eligible compounds, 2-hydroxy-l-naphthaldehyde 
Table 28. Reagents used to detect hydrazine after generation from $\underset{\sim}{2}$ on a t.l.c. plate

Reagent

$p-\mathrm{Et}_{2} \mathrm{NC}_{6} \mathrm{H}_{4} \mathrm{CHO}^{\mathrm{a}}$

$p-\mathrm{Me}_{2}{ }^{\mathrm{NC}}{ }_{6} \mathrm{H}_{4} \mathrm{CH}=\mathrm{CHCHO}^{\mathrm{a}}$

$0-\mathrm{HOC}_{6} \mathrm{H}_{4} \mathrm{CHO}^{\mathrm{a}}$

$\mathrm{OHCCH}=\mathrm{CHCH}_{2} \mathrm{CHO}^{\mathrm{b}}$

$p-\mathrm{Me}_{2} \mathrm{~N}-\mathrm{O}-\mathrm{HOC}_{6} \mathrm{H}_{3} \mathrm{CHO}^{\mathrm{a}}$

Picryl chloride ${ }^{a}$

2-Nitro-l, 3-

indandione ${ }^{a}$

Ammoniacal silver
References

98

99,100

101

120

50

102

$<500$

103

$<500$

104 25

25

10
Limit of Visibilization on detection the t.l.c. plate of $\underset{\sim}{2}(n g)$

a faint yellow spot on a colorless background

with a small amount, a dark spot on a yellow background; in larger quantity, the spot is red

under the 366-nm lamp, with liquidnitrogen cooling, a faint pink spot on a blue background red spot on a yellow background under visible light, light yellow on a colorless background; under the 366-nm lamp, pink on a maroon background; no color enhancement on liquidnitrogen cooling orange spot on a brown background

no distinct color; light spot on a tan background

brown spot on a gray background

${ }^{a}$ Reagent used was $1 \%$, in absolute alcohol solution.

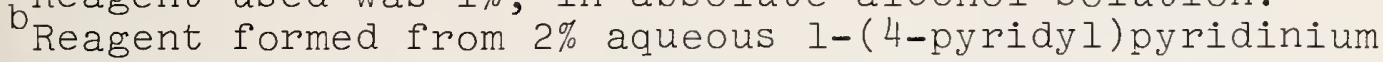
chloride solution by mixing with an equal volume of $2 M$ NaOH solution immediately before spraying.

Formed in the usual way for preparing the Tollens reagent. 
appears the most promising of those tested thus far. By using this aldehyde, $\underset{\sim}{2}$ can be detected on a t.l.c. plate at the 50-ng level. Experimentation is in progress to determine whether the 2,2'-dihydroxy-1, I'-napthalazine can be extracted from a t.l.c. plate and the fluorescence spectrum of the extract measured without interference.

\section{Detection of 2 by the Franchimont Test}

(W. J. Rossiter, Jr.)

The Franchimont test [106] is an analytical colorreaction specific to the $N$-nitro group both of $N$-nitroamines and $N$-nitroamides. The basis of this test is that the $N-$ nitro compound produces a characteristic color, usualy violet, when added to a mixture of zinc and acetic acid in the presence of certain aromatic amines. N-Nitroso compounds produce no color under identical conditions.

We have adapted this test to the low-level detection of 2. The test, as employed for our purposes, has some similarity to the preceding method of detecting 2 through hydrazine formation. Thus, the zinc that is required for the test is incorporated into the silica gel used for coating the thin-layer plate, and acetic acid is used as the vapor. However, the difference between the two methods of detecting 2 is that, in the Franchimont test, the t.l.c. plate is sprayed with an alcoholic solution of an aromatic amine and dried before immersion in the acetic acid vapor. The amine used was 2-naphthylamine.

Our adaptation of the Franchimont test is conducted in the following simple way. A quantity of 2 is spotted on a t.l.c. plate precoated with Silica Gel GF-254 containing 5\% of zinc dust (by weight). The plate is sprayed, until wet, with a 1\% alcoholic solution of 2-naphthylamine, and, after partial air-drying, the plate is placed in an atmosphere of acetic acid vapor, where it is allowed to remain for $30 \mathrm{~min}$ 
before being removed. During this time, if a sufficient quantity of $\underset{\sim}{2}$ is present, yellow spots start to appear. At the end of the 30-min reaction period, spots of 2 in the $2.0-$

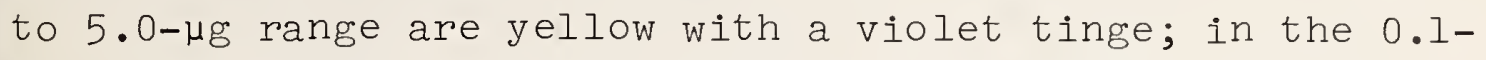
to $1.0-\mu \mathrm{g}$ range, they are pale yellow; and, below $0.1 \mu \mathrm{g}$, they cannot be observed in ordinary, visible-wavelength light. However, under the 366-nm lamp, spots representing quantities of $\underset{\sim}{2}$ as low as $5 \mathrm{ng}$ can be observed by fluorescence. At the 5-ng level, the spot is a pale, light blue on a deeper blue background. As the amount of $\underset{\sim}{2}$ is increased, the intensity of the fluorescence increases, but there is no apparent increase in color intensity when the t.l.c. plate is immersed in liquid nitrogen.

other aromatic amines have been examined in this t.l.c. version of the Franchimont test. Many produce a yellow to violet-yellow spot on the t.l.c. plate. However, none tested has produced a fluorescent spot. Thus far, only with 2-naphthylamine in this test has this low level of detection of $\underset{\sim}{2}$ been achieved.

Little seems to be known about the mechanism of the Franchimont test [107]. It is possible that such knowledge would aid in its further exploitation.

\section{Spectrodensitometric Means of Detecting Low Levels of 2 \\ (A. Cohen)}

Spectrodensitometric methods for detecting $\underset{\sim}{2}$ at low levels were examined. This procedure affords the possibility of detecting the compound without the destruction that occurs when spray reagents are used, and thus, of recovering the compound for further identification. Several commercial densitometers were used in the examination of 250-um thick, silica gel plates, fluorescent and nonfluorescent, that had been spotted with $30-60$ ng of compound $\underset{\sim}{2}$, developed, and dried. The plates were examined by $u \cdot v$. absorption and by 
fluorescence quenching-reflectance methods. Usually, a larger amount of the compound had to be spotted also, in order to determine the approximate region where the soughtfor trace of the compound might be. Its exact location might still require mm-by-mm examination of the region.

The smallest amount of compound detectable with a spectrodensitometer was at the 30-ng level. To detect this amount requires high instrument-gain and/or recorderexpansion. Hence, background noise is substantial. A major part of this high background may result from imperfections in the adsorbent layer or, possibly, unequal distribution of the fluorescing material in the adsorbent. These imperfections may limit the usefulness of this technique. 
A. Personnel Listing

R. Schaffer, Section Chief

R. S. Tipson

B. Coxon

A. J. Fatiadi

R. F. Brady, Jr.

W. J. Rossiter, Jr.

B. F. West

A. Cohen

J. H. Thomas

B. A. Johnson

D. W. Vomhof, Research Associate to 9-26-69

D. Niner, Guest Worker (1969)

J. James, Guest Worker (1970)

\section{B. Publications}

NBS Technical Note 507. Organic Chemistry Section. $7 / 1 / 68-6 / 30 / 69$.

Edited by R. Schaffer (October, 1969).

Synthesis of the Two D-2-Pentuloses. New Derivatives of D-erythro-Pentulose.

R. S. Tipson and R. F. Brady, Jr., Carbohyd. Res. 10,

549 (1969).

Studies of ${ }^{15}$ N-Labeled Amino Sugars. Synthesis and Spectroscopy of Derivatives of 6-Amino-6-deoxy-Dglucose-6-15N.

B. Coxon, Carbohyd. Res. 11, 153 (1969).

Review of Organic Structure Determination, by D. J. Pasto and C. R. Johnson.

B. Coxon, Capital Chemist 19, 143 (1969).

Review of Organic Synthesis, by R. E. Ireland.

R. F. Brady, Jr., Capital Chemist 19. 239 (1969).

Phenylhydrazono-phenylazo Tautomerism. Part III.

Reactions of Phenylformazans and Certain

Bis (phenylhydrazones) with Strong Acids.

H. S. Isbell and A. J. Fatiadi, Carbohyd. Res. II,

303 (1969). 
A Comparison of the Spectrophotometric and Gas-

chromatographic Methods with the Vacuum-oven Procedure

for the Determination of Moisture in Starch Hydrolyzates.

D. W. Vomhof and J. H. Thomas, Ann. Proc. Am. Soc.

Brewing Chemists, 1969, 139.

Novel Aromatization of a Trihydroxycyclohexanetrione (Triketoinositol) to a Dibenzo-p-dioxin Derivative.

A. J. Fatiadi, Carbohyd. Res. I2, 130 (1970).

Preparation of Inositol Hexasulfate.

A. J. Fatiadi, Carbohyd. Res. 12, 293 (1970).

Novel Iodination of the $\alpha$-Nethylene Group in 1,3Diphenylpropane-1,3-dione and Related B-Diketones with Periodic Acid.

A. J. Fatiadi, Chem. Commun. 1970, 11.

Conversion of Certain Cyclic Phenylhydrazino Derivatives into Phenylazo Compounds with Periodic Acid.

A. J. Fatiadi, J. Org. Chem. 35, 831 (1970).

The Synthesis, Equilibration, and Conformations of Diastereoisomeric 1,2-0-Isopropylidene-3,5-0-(methoxy-

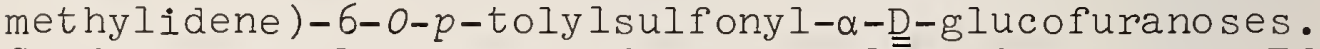
Conformational Evidence from a Nuclēar Overhauser Effect.

B. Coxon, Carbohyd. Res. 12, 313 (1970).

Review of The Amino Sugars. The Chemistry and Biology of Compounds Containing Amino Sugars; Volume IA, Chemistry of Amino Sugars, edited by R. W. Jeanloz.

B. Coxon, Carbohyd. Res. 13, 319 (1970).

Model Parameters for the Analysis of Skew Conformations of Carbohydrates by P.M.R. Spectroscopy .

B. Coxon, Carbohyd. Res. 13, 321 ( 1970 ).

Review of Biokhimiya. Uglevody. Uspekhi v Izuchenii Stroeniya i Metabolisma, by B. N. Stepanenko.

A. J. Fatiadi, Carbohyd. Res. 13, 472 (1970).

C. Manuscripts in Process of Publication

Isotopic Methods in Carbohydrate Chemistry.

R. Schaffer. (Manuscript accepted for "The

Carbohydrates," edited by W. Pigman and D. Horton,

Chapter 45, Vol. 2B, in press)

Occurrence, Properties, and Preparation of Naturally Occurring Monosaccharides, Including 6-Deoxy Sugars.

R. Schaffer. (Manuscript accepted for "The

Carbohydrates," edited by W. Pigman and D. Horton,

Vol. IA, in press) 
Infrared Spectroscopy of Carbohydrates.

R. S. Tipson and F. S. Parker. (Manuscript accepted

for "The Carbohydrates," edited by W. Pigman and

D. Horton, Vol. 1B, in press)

Conformational Analysis via Nuclear Magnetic Resonance Spectroscopy

B. Coxon. (Manuscript accepted for "Methods in

Carbohydrate Chemistry," edited by R. L. Whistler

and J. N. BeMiller, Vol. 6, in press)

The Conformations of $1,2: 4,6-D i-0-b e n z y l i d e n e-\alpha-D-$ glucopyranose Derivatives.

B. Coxon. (Manuscript accepted for publication in

Carbohydrate Research)

Electron-spin Resonance Study of an Alkaline Solution of Copper(II) Oxalate-meso-Tartrate Complex (the Somogyi Reagent) and Related Complexes.

A.J. Fatiadi. (Manuscript accepted for publication

in J. Res. NBS )

Cyclic Acetals of Ketoses. Part III. Re-investigation of the Synthesis of the Isomeric Di-0-isopropylidene-Dfructopyranoses.

R. F. Brady, Jr. (Manuscript accepted for publication

in Carbohydrate Research)

Cyclic Acetals of Ketoses. Part IV. Re-investigation of the Oxidation of $1,2: 4,5-D i-0-i$ sopropylidene- $\beta$-Dfructopyranose with Methyl Sulfoxide-Acetic Anhydride. R. S. Tipson, R. F. Brady, Jr., and B. F. West.

(Manuscript accepted for publication in Carbohydrate Research)

Determination of Moisture in Starch Hydrolyzates by Near-infrared and Infrared Spectrophotometry .

D. W. Vomhof and J. H. Thomas. (Manuscript accepted

for publication in Analytical Chemistry)

Determination of Moisture in Syrups by Gas-Solid

Chromatography .

D. W. Vomhof and J. H. Thomas. (Manuscript submitted

for publication in J. Ass. Offic. Anal. Chem.)

Determination of Inososes with an Alkaline Solution of Copper(II) Oxalate-Tartrate Complex (the Somogyi Reagent); and Reaction Mechanisms Involved.

A. J. Fatiadi. (Manuscript submitted for publication

in Carbohydrate Research)

Evidence for Adsorption as the First step in the Solidstate Oxidation of Benzenehexol with Active Manganese Dioxide.

A. J. Fatiadi. (Manuscript completed) 
D. Lectures

$9 / 8 / 69$ - Studies of ${ }^{15} \mathrm{~N}$-Labeled Amino Sugars. Synthesis and Spectroscopy of Derivatives of 6-Amino-6-

deoxy-D-glucose-6- ${ }^{15} \mathrm{~N}$. Carbohydrate Division, l58th Ämerican Chemical Society Meeting, New York, New York. B. Coxon.

12/4/69-Chemistry of Keto Sugars. Student Affiliate Chapter of the American Chemical Society, Mount Saint Mary's College, Emmitsburg, Maryland. R. F. Brady, Jr.

12/17/69-The Aldol Reaction in Carbohydrate Chemistry. Chemistry Department, American University, Washington, D. C. R. Schaffer.

3/18/70-Current Status of Clinical Standard Reference Materials. Northeast Section of the American Association of Clinical Chemists, Boston, Massachusetts. R. Schaffer.

3/19/70-Current Status of Clinical standard Reference Materials. Clinical Chemistry Laboratory, Hartford Hospital, Hartford, Connecticut. R. Schaffer.

3/23/70-Bilirubin Standard Reference Material. 5th Annual Meeting of the Association for the Advancement of Medical Instrumentation, Boston, Massachusetts. R. Schaffer.

5/27/70-Experiences with Computerized, Iterative Analysis of the N.M.R. Spectra of Carbohydrates. Joint ACS-CIC Meeting, Toronto, Ontario, Canada. B. Coxon. 
6. ACKNOWLEDGMENTS

The editor gratefully acknowledges the cooperation of his associates in the Organic Chemistry section in the preparation of this report. Special thanks are due Dr. R. S. Tipson for facilitating the editorial effort. Miss Barbara Hyde, Section Secretary, brought the manuscript to final form. We wish also to thank Mrs. R. S. Maddock, Administrative Assistant for the Division of Analytical Chemistry in Charge of Publications, for assisting in its publication. 


\section{REFERENCES}

[1] Brady, R. F., Jr., NBS Technical Note 457, 88, 90 (1968).

[2] Brady, R. F., Jr., NBS Technical Note 507. 97 (1969).

[3] Clin. Chem. 8, 405 (1962).

[4] Gambino, S. R., Standard Methods of Clin. Chem. 5, 55 (1965).

[5] Gadd, K. G., J. Clin. Pathol. 19, 300 (1966).

[6] Billing, B. H., Halsam, R., Wald, A., personal communication.

[7] Dybkaer, R., Hertz, H., Scand. J. Clin. Lab. Invest. 25, 151 (1970).

[8] Henry, R. J., Clinical Chemistry: Principles and Techniques, Harper and Row, New York, 1967, p. 584.

[9] Coxon, B., NBS Technical Note 507, 79 (1969).

[10] Ono, S., Takahashi, K., in Biochemical Calorimetry, Brown, D. H., (Ed.) Academic Press, New York, 1969, p. 101.

[11] Vomhof, D. W., Thomas, J. H., NBS Technical Note 507, 111 (1969); Currie, L., Anal. Chem. 40, 586 (1968).

[12] The Merck Index, Eighth Edition, Merck \& Co., Inc., Rahway, N. J., 1968, p. 542.

[13] Hayden, A. L., Anal. Chem. 27, 1486 (1955).

[14] Goodman, I., Olenczak, A. P., Scherrer, J.W., Hiatt, R. B., Biochim. Biophys Acta 156, 364 (1968).

[15] Richtmyer, N. K., Hudson, C. S., J. Amer. Chem. Soc. 13, 2249 (1951).

[16] P-L Biochemicals, Inc., Reference Guide Catalog No. 102, Milwaukee, Wisconsin, 1970, p. 11.

[17] Burton, R. M., Pietro, A. S., Arch. Biochem. Biophys. 48, 184 (1954).

[18] Dalziel, K., Biochem. J. 84, 240 (1962).

[19] Dalziel, K., Dickinson, F. M., Biochem. Prepn. II, 84 (1966).

[20] Kaplan, N. O., Colowick, S. P., Barnes, C. C., J. Biol. Chem. 191, 461 (1951).

[21] Huff, J.W., Perlzweig, W. A., J. Biol. Chem. 167, 157 (1947). 
[22] Specifications and Criteria for Biochemical Compounds, Tipson, R. S., (Ed.) Publication 1344, National Academy of Sciences-National Research Council, Washington, D. C., 1967, CoE-7, p. 207.

[23] Worthington Biochemical Corp., Freehold, New Jersey.

[24] Fischer, E., Ber. 28, 1145 (1895).

[25] Bell, D. J., J. Chem. Soc. 1947, 1461.

[26] Ohle, H., Koller, I., Ber. 57, 1566 (1924).

[27] Tipson, R. S., West, B. F., Brady, R. F., Jr., Carbohya. Res. 10, 181 (1969).

[28] Brady, R. F., Jr., Carbohyd. Res. (1970) in press.

[29] Eble, T. E., Hoeksema, H., Boyack, G. A., Savage, G. M., Antibiot. Chemotherapy 2, 419 (1959); Vavra, J. J., Dietz, A., Churchill, B. W., Smirnoff, P., Koepsell, H. J., ibid. 2, 427 (1959); Schroeder, W., Hoeksema, H., J. Amer. Chem. Soc. 81, 1767 (3959); Garrett, E. E., ibid. 82, 827 (1960); Farkas, J., Śrm, F., Collect. Crech. Chem. Commun. 28, 882 (1963).

[30] Hough, L., Stacey, B. E., Phytochemistry 2, 315 (1963); 5, $171,215(1966)$.

[31] Zerban, F.W., J.Ass. Offic. Agr. Chem. 24, 656 (1941); Zerban, F. W., Sattler, L., J.Amer. Chem. Soc. 64, 1740 (1942); Ind. Eng. Chem. 34, 1180 (1942).

[32] Steiger, M., Reichstein, T., Helv. Chim. Acta 19, 184 $(1936)$.

[33] Wolfrom, M. L., Thompson, A., Evans, E. F., J. Amer. Chem. Soc. 67, 1793 (1945).

[34] Jones, J.K. N., Nicholson, W. H., J. Chem. Soc. 1955, 3050 .

[35] Lobry de Bruyn, C. A., Alberda van Ekenstein, W., Rec. Trav. Chim. 14, 195 (1895); de Whalley, H.C.S., Albon, N., Gross, D., Analyst 76, 287 (1951); Schneider, F., Erlemann, G. A., Zucker-Beih. 3, 41 (1951); Zerban, F. W., Sattler, L., Rosenthal, G., Glaubach, A., Sugar 47, [2] 33 (1952); Hough, L., Jones, J. K. N., Richards, E. L., J. Chem. Soc. 1953, 2005.

[36] Passeron, S., Recondo, E., J. Chem. Soc. 1965, 813.

[37] McDonald, E. J., Carbohyd. Res. 5, 106 (1967).

[38] Takagi, S., Rosenstein, R. D., Acta Crystallogr. A25, [S3] S197 (1969); Carbohyd. Res. 11, 156 (1969); personal communication, February 24 th, 1970. 
[39] James, K., Tatchell, A. R., Ray, P. K., J. Chem. Soc. (C) $1967,2681$.

[40] Tipson, R. S., West, B. F., NBS Technical Note 457 , $41(1968)$.

[41] Cree, G. M., Perlin, A. S., Can. J. Biochem. 46. 765 (1968).

[42] Fischer, E., Noth, H., Ber. 51, 321 (1918).

[43] Lawton, B. T., Szarek, W. A., Jones, J. K. N., Carbohyd. Res. 10, 456 (1969).

[44] Maeda, T., Tori, K., Satoh, S., Tokuyama, K., Bull. Chem. Soc. Jap. 42, 2635 (1969).

[45] Hudson, C. S., Brauns, D. H., J. Amer. Chem. Soc. 38 , 1222 (1916).

[46] Tipson, R. S., Brady, R. F., Jr., West, B. F., Carbohyd. Res. (1970) in press.

[47] Fletcher, H. G., Jr., Ness, R. K., J.Amer. Chem. Soc. 77, 5337 (1955).

[48] Coxon, B., Carbohyd. Res. 8, 125 (1968).

[49] Hendrickson, J. B., J. Org. Chem. 29, 991 (1964).

[50] Coxon, B., Tetrahedron 22, 2281 (1966).

[51] Sternhell, S., Rev. Pure Appl. Chem. 14, 15 (1964).

[52] Sternhell, S., Quart. Rev. (London) 23, 236 (1969).

[53] Barfield, M., J. Chem. Phys. 4I, 3825 (1964).

[54] Hall, L. D., Manville, J. F., Carbohyd. Res. 8 , 295 (1968).

[55] Bystrov, V.F., Stepanyants, A. U., J. Mol. Spectrosc. 21, 241 (1966).

[56] Coxon, B., Carbohyd. Res. 13, 321 (1970).

[57] Wood, H. B., Jr., Diehl, H.W., Fletcher, H. G., Jr., J. Amer. Chem. Soc. 79 , 1986 (1957).

[58] Castellano, S., Bothner-By, A. A., J. Chem. Phys. 4I, $3863(1964)$.

[59] Karplus, M., J. Amer. Chem. Soc. 85, 2870 (1963).

[60] Baggett, N., Duxbury, J.M., Foster, A. B., Webber, J. M., Chem. Ind. (London) 1964, 1832.

[61] Baggett, N., Buck, K. W., Foster, A. B., Webber, J. M., J. Chem. Soc. 1965, 3401 .

[62] Coxon, B., Carbohyd. Res. (1970), in press.

[63] Coxon, B., Carbohyd. Res. 12, 313 (1970). 
[64] Coxon, B., NBS Technical Note 507, 23 (1969).

[65] Ref. 64, p. 21.

[66] Coxon, B., Carbohyd. Res. 11, 153 (1969).

[67] Bothner-By, A. A., Castellano, S. M., LAoCN3, a publication of the Mellon Institute, Pittsburgh, $\mathrm{Pa}$. (1966).

[68] Johannesen, R. B., Ferretti, J. A., Harris, R. K., J. Mag. Res. (1970), in press.

[69] Dunhill, R. H., Pilbrow, J. R., Smith, T. D., J. Chem. Phys. 45, 1474 (1966); Boss, J. F., Dunhill, R.H., Pilbrow, J. R., Srivastave, R. C., Smith, T. D., J. Chem. Soc. (A) 1969, 94.

[70] Belford, R. L., Chasteen, N. D., So, H., Tapscott, R. E., J. Amer. Chem. Soc. 91, 4675 (1969); Chasteen, N. D., Belford, R. L., Paul, I. C., Inorg. Chem. 408 (1969).

[71] Chasteen, N. D., Belford, R. L., Inorg. Chem. 9, 169 (1970).

[72] Cotton, F. A., Wilkinson, G., Advanced Inorganic Chemistry, 2nd edition, Interscience Publishers, New York, 1966, p. 906.

[73] Rajan, K. S., Martell, A. E., J. Inorg. Nucl. Chem. 29, 463 (1967).

[74] Kivelson, D., J. Chem. Phys. 45, 1324 (1966).

[75] Solozhenkin, P. M., Kopitsya, N. I., J. Structur. Chem. \&, 470 (1967).

[76] Fatiadi, A. J., Carbohyd. Res. 12, 293 (1970).

[77] Fatiadi, A. J., J. Org. Chem. 35, 831 (1970).

[78] Fatiadi, A. J., Chem. Commun. 1967, 1087; J. Res. NBS 72A, 341 (1968).

[79] Park, J. D., Brown, H. A., Lacher, J. R., J. Amer. Chem. Soc. 75, 4753 (1953); Combes, A., Compt. Rend. $111,273(1890)$.

[80] Djerassi, C., Lenk, C. T., J. Amer. Chem. Soc. 75, 3494 (1953); Djerassi, C., Grossman, J., Thomas, G. H., ibid. 77, 3826 (1955).

[81] Fieser, L. F., Fieser, M., Reagents for Organic Synthesis, John Wiley, New York, 1967, pp. 500-511.

[82] Pauling, L., The Nature of the Chemical Bond, Cornell University Press, Ithaca, New York, 1948, Chapter 7.

[83] Fatiadi, A. J., Chem. Commun. 1970, ll. 
[84] Rüdiger, W., Klose, W., Tetrahedron Lett. 47. 5893 (1966).

[85] Rüdiger, W., Z. Physiol. Chem. 348, 129 (1967).

[86] Rüdiger, W., Klose, W., Vuillanme, M., Barbier, M., Experientia 24, 1000 (1968).

[87] Fischer, H., Rose, H., Ber. 45, 1579 (1912).

[88] Fatiadi, A. J., Chem. Commun. 1968, 456.

[89] Cohen, A., NBS Technical Note 507, 109 (1969).

[90] Szent-Gyorgi, A., Science 126, 751 (1957).

[91] Snell, F. D., Snell, C. T., Colorimetric Methods of Analysis, Van Nostrand, New York, Vol. 1, 1936, p. 639 .

[92] Fiegl, F., Spot Tests in Organic Analysis, Elsevier, New York, 1966, p. 300; Bose, P. K., Analyst 56, 504 (1931).

[93] Vernazza, N. E., Acad. Sci. Torino, Classe Sci. Fis. Mat. Nat. 70, 404 (1935).

[94] Sawicki, E., Stanley, T. W., Pfaff, J., Chemist-Analyst 51, 9 (1962).

[95] Civera, M., Gazz. Chim. Ital. 86, 139 (1956).

[96] Duden, P., Scharff, M., Ann. 288, 239 (1895).

[97] Thawley, A. R., J. Chromatogr. 38, 399 (1968).

[98] Pesez, M., Petit, A., Bull. Soc. Chim. Fr. 1947, 122.

[99] Feigl, A., Manheimer, W. A., Mikrochem. Mikrochim. Acta 40,50 (1952).

[100] Kul'berg, L. M., Il'ina, T. S., Ukr. Khim. Zh. 2l, 97 (1955).

[101] Anger, V., Ofri, S., Mikrochim. Acta 1964, 626.

[102] Kul'berg, L. M., Cherkesov, A. I., Zh. Anal. Khim. 6, 364 (1951).

[103] Vanags, G., Mackanova, M., J. Gen. Chem. USSR 25, 551 (1955).

[104] Sant, B. R., Mikrochim. Acta 1958, 169.

[105] Feigl, F., Spot Tests in Organic Analysis, Elsevier, New York, 1966, p. 341.

[106] Franchimont, A. P. N., Rec. Trav. Chim. 16, 227 (1897).

[107] Smith, P. A. S., The Chemistry of Open-chain Organic Nitrogen Compounds, Benjamin, New York, 1966, Vol. 2, p. 497. 
Latest developments in the subject area of this publication, as well as in other areas where the National Bureau of Standards is active, are reported in the NBS Technical News Bulletin. See following page. 


\section{HOW TO KEEP ABREAST OF NBS ACTIVITIES}

Your purchase of this publication indicates an interest in the research, development, technology, or service activities of the National Bureau of Standards.

The best source of current awareness in your specific area, as well as in other NBS programs of possible interest, is the TECHNICAL NEWS BULLETIN, a monthly magazine designed for engineers, chemists, physicists, research and product development managers, librarians, and company executives.

If you do not now receive the TECHNICAL NEWS BULLETIN and would like to subscribe, and/or to review some recent issues, please fill out and return the form below.

Mail to: Office of Technical Information and Publications National Bureau of Standards

Washington, D. C. 20234

Name

Affiliation

Address

City State Zip

Please send complimentary past issues of the Technical News Bulletin.

Please enter my 1-yr subscription. Enclosed is my check or money order for $\$ 3.00$ (additional $\$ 1.00$ for foreign mailing). check is made payable to: SUPERINTENDENT OF DOCUMENTS. TN547 


\section{PERIODICALS}

JOURNAL OF RESEARCH reports National Bureau of Standards research and development in physics, mathematics, chemistry, and engineering. Comprehensive scientific papers give complete details of the work, including laboratory data, experimental procedures, and theoretical and mathematical analyses. Illustrated with photographs, drawings, and charts.

Published in three sections, available separately:

\section{- Physics and Chemistry}

Papers of interest primarily to scientists working in these fields. This section covers a broad range of physical and chemical research, with major emphasis on standards of physical measurement, fundamental constants, and properties of matter. Issued six times a year. Annual subscription: Domestic, $\$ 9.50$; foreign, $\$ 11.75^{*}$.

\section{Mathematical Sciences}

Studies and compilations designed mainly for the mathematician and theoretical physicist. Topics in mathematical statistics, theory of experiment design, numerical analysis, theoretical physics and chemistry, logical design and programming of computers and computer systems. Short numerical tables. Issued quarterly. Annual subscription: Domestic, $\$ 5.00$; foreign, $\$ 6.25 *$.

\section{- Engineering and Instrumentation}

Reporting results of interest chiefly to the engineer and the applied scientist. This section includes many of the new developments in instrumentation resulting from the Bureau's work in physical measurement, data processing, and development of test methods. It will also cover some of the work in acoustics, applied mechanics, building research, and cryogenic engineering. Issued quarterly. Annual subscription: Domestic, $\$ 5.00$; foreign, $\$ 6.25^{*}$.

\section{TECHNICAL NEWS BULLETIN}

The best single source of information concerning the Bureau's research, developmental, cooperative and publication activities, this monthly publication is designed for the industry-oriented individual whose daily work involves intimate contact with science and technology-for engineers, chemists, physicists, research managers, product-development managers, and company executives. Annual subscription: Domestic, $\$ 3.00$; foreign, $\$ 4.00^{*}$.

\section{NONPERIODICALS}

Applied Mathematics Series. Mathematical tables, manuals, and studies.

Building Science Series. Research results, test methods, and performance criteria of building materials, components, systems, and structures.

Handbooks. Recommended codes of engineering and industrial practice (including safety codes) developed in cooperation with interested industries, professional organizations, and regulatory bodies.

Special Publications. Proceedings of NBS conferences, bibliographies, annual reports, wall charts, pamphlets, etc.

Monographs. Major contributions to the technical literature on various subjects related to the Bureau's scientific and technical activities.

National Standard Reference Data Series. NSRDS provides quantitative data on the physical and chemical properties of materials, compiled from the world's literature and critically evaluated.

Product Standards. Provide requirements for sizes, types, quality and methods for testing various industrial products. These standards are developed cooperatively with interested Government and industry groups and provide the basis for common understanding of product characteristics for both buyers and sellers. Their use is voluntary.

Technical Notes. This series consists of communications and reports (covering both other agency and NBS-sponsored work) of limited or transitory interest.

Federal Information Processing Standards Publications. This series is the official publication within the Federal Government for information on standards adopted and promulgated under the Public Law 89-306, and Bureau of the Budget Circular A-86 entitled, Standardization of Data Elements and Codes in Data Systems.

- Difference in price is due to extra cost of foreign mailing.

Order NBS publications from:

Superintendent of Documents

Government Printing Office

Washington, D.C. 20402 
U.S. DEPARTMENT OF COMMERCE

WASHINGTON, D.C. 20230

\section{OFFICIAL BUSINESS}

PENALTY FOR PRIVATE USE, $\$ 300$

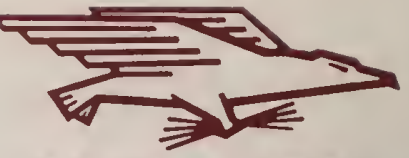

POATAGE AND FEES PAID U.8. DEPARTMENT OF COMMERCE 